\title{
Auto-Tune's Effect on Musicians, Genres, and Culture
}

............

by

Matthew McGowan

A Thesis submitted to the Faculty of Graduate Studies and Research in partial fulfillment of the requirements for the degree of

\section{Master of Arts}

in

Music and Culture

Carleton University

OTTAWA, Ontario

May 14, 2012

(C) 2012

Matthew McGowan 
Library and Archives

Canada

Published Heritage

Branch

395 Wellington Street

Ottawa ON K1A ON4

Canada
Bibliothèque et

Archives Canada

Direction du

Patrimoine de l'édition

395 , rue Wellington

Ottawa ON K1A ON4

Canada
Your file Votre référence

ISBN: 978-0-494-91567-7

Our file Notre référence

ISBN: 978-0-494-91567-7
NOTICE:

The author has granted a nonexclusive license allowing Library and Archives Canada to reproduce, publish, archive, preserve, conserve, communicate to the public by telecommunication or on the Internet, loan, distrbute and sell theses worldwide, for commercial or noncommercial purposes, in microform, paper, electronic and/or any other formats.

The author retains copyright ownership and moral rights in this thesis. Neither the thesis nor substantial extracts from it may be printed or otherwise reproduced without the author's permission.
AVIS:

L'auteur a accordé une licence non exclusive permettant à la Bibliothèque et Archives Canada de reproduire, publier, archiver, sauvegarder, conserver, transmettre au public par télécommunication ou par l'Internet, prêter, distribuer et vendre des thèses partout dans le monde, à des fins commerciales ou autres, sur support microforme, papier, électronique et/ou autres formats.

L'auteur conserve la propriété du droit d'auteur et des droits moraux qui protege cette thèse. $\mathrm{Ni}$ la thèse ni des extraits substantiels de celle-ci ne doivent être imprimés ou autrement reproduits sans son autorisation.
In compliance with the Canadian Privacy Act some supporting forms may have been removed from this thesis.

While these forms may be included in the document page count, their removal does not represent any loss of content from the thesis.
Conformément à la loi canadienne sur la protection de la vie privée, quelques formulaires secondaires ont été enlevés de cette thèse.

Bien que ces formulaires aient inclus dans la pagination, il n'y aura aucun contenu manquant. 


\section{Abstract}

In this thesis, I will examine the vocal effect technology Auto-Tune, a piece of software used in modern recording studios to alter the pitch of a singer's recorded vocal performance, or to generate a unique, overt vocal effect. While Auto-Tune has generally been implemented successfully within the pop music genre, the use of Auto-Tune within modern hip hop has resulted in a contentious discourse within said genre.

This thesis shall explore not only how Auto-Tune is utilized within popular music, but also how it impacts the way musicians record and perform music, how Auto-Tune either complements or conflicts with the aesthetic foundations and conventions of a given genre, what symbolic effect Auto-Tune has upon the musical recording it is applied to (according to a variety of theoretical notions), and what influence Auto-Tune has had upon the broader popular culture. 


\section{Acknowledgements}

Professionally, I would like to deeply thank Professor William Echard for agreeing to supervise my thesis. His calm, professional, and helpful character was of great personal reassurance and confidence to me during the course of researching, writing, editing, and defending my work. As well, his incredible depth of academic knowledge, as well as expertise in the technological aspect of music, proved to be an indispensable resource.

As well, I would like to thank Professor James Deaville for his guidance and counsel as Graduate Supervisor over the course of my studies at Carleton University. Also, thanks go out to Barbara Shannon, the Graduate Administrator of the School for Studies in Art and Culture, for being equally helpful and knowledgeable in all administrative matters.

Personally, I would like to thank my wife Lindsay. Without your unwavering and selfless love and support, I simply would not have been able to complete this thesis. I cannot thank you enough. 
I - Introduction 1

II - Synthesis, Sampling, and Digital Signal Processing 6

Moog Modular Synthesizer and Synthesis 7

Digital Synthesizers, Samplers and Processors 9

Digital Audio and Quantization 14

Pro-Tools 16

Auto-Tune 20

Conclusion 25

III - Auto-Tune Within Popular Music 28

Pop Music 29

Hip Hop 38

Hip-Pop 54

Conclusion 61

IV - Popular Music Vocal Aesthetics and Auto-Tune 63

Popular Music vs. Pop Music 64

Pop Music 66

Pop Music and Technology 69

Old-School Hip Hop Vocal Aesthetics 76

Hip-Pop Aesthetics 80

Conclusion 86

V - Theory 90

Naomi Cumming and The Sonic Self 91

Roland Barthes and The Grain of the Voice 102

Jean Baudrillard and The Hyperreal 106

Jonathan Sterne and Schizophonia 111 
Conclusion 115

VI - Conclusion: Auto-Tune and Popular Culture 116

Television 117

Internet 121

Music Technology 126

Popular Music 129

Bibliography 134

Websites 137

Online Multimedia 138

Discography 139 


\section{I - Introduction}

According to musical theory, there exist a plethora of tuning systems, such as Just Intonation, Pythagorean Tuning, Meantone Temperament, and others. However, the Twelve Tone Equal Temperament (12-TET) tuning system has managed to become the dominant standard of intonation within almost all western music for a variety of reasons, such as its technical ability to achieve perfectly consistent tuning between all twelve chromatic tones, to enable the composer to transpose freely between keys, and also because it is what musicians are taught, instruments are designed to play, and audiences have become accustomed to. As a western musical audience, we are culturally conditioned towards a preference for particular musical tunings, such as 12-TET, and thus engrain these preferences by espousing them as the dominant, preferred tuning system amongst nearly all the genres in the western world.

While the 12-TET system provides the aesthetic backbone for nearly all forms of western music, there are instances where alternative systems and/or aesthetics of intonation come into play. For example, within African-American (i.e. blues and jazz) and European folk music, the blue note -- in which certain notes are sung at a slightly lower pitch relative to 12-TET -- is a technique used for expressive purposes. These notes are used by musicians to create a certain tonal dissonance and tension, one which (in the case of the blues) allows the musician to evoke a certain human, emotional quality (through either the musician's instrument or the singer's voice). Bessie Smith's “Nobody Knows You 
When You Are Down and Out"1 is an apt example of a vocalist using the blue note, possibly to elicit emotions such as loss, sorrow, regret, and resilience by phrasing the pitch of her vocals slightly lower than would be expected under 12TET. The blue note is a prime example of a non-12-TET tuning aesthetic having been imported into a style of music -- such as African American blues and jazz, European folk, etc., -- with great acceptance and success, by appealing to the aesthetic preferences of the musicians and audiences of these particular genres, while also providing new techniques of phrasing their performances in such a way as to elicit new and evocative musical expressions.

However, the introduction of new tuning systems and aesthetics into a musical genre is not always successful: in instances where two or more contradictory tuning systems and/or aesthetic preferences come into conflict, a power struggle can ensue over which system or aesthetic will be regarded as either the dominant or most influential system within the genre. Recently, certain engrained values and aesthetic preferences regarding 12-TET within popular music have been highlighted by the use of Auto-Tune, a post production vocal editing software suite which allows musicians, engineers, and producers to either clandestinely edit the pitch of a musician's recorded vocals (bringing the singer's vocals not only in-tune but pitch-perfect), or to more overtly effect the singer's voice, producing a wildly oscillating digital tremolo in the vocal. While Auto-Tune challenges the tuning systems and aesthetics of both pop music and hip hop, in

\footnotetext{
1 Bessie Smith, "Nobody Knows You When You're Down and Out," The Essential Bessie Smith, Sony Imports B000002ADO, compact disc, 2006, originally released in 1929.
} 
the latter case it primarily produces a conflict, power struggle, or resistance. Currently, within hip hop, there is an ongoing conflict over the use of Auto-Tune, between an established old-school sect who oppose its import into hip hop, and a newer, younger cadre of artists who are more open to not only Auto-Tune, but also to other pop music sensibilities. What is at stake in this conflict is not only the dominant sound (or tuning system) of hip hop, but the essence of hip hop as a sample-based, rapped verse form of music, versus an increasingly Auto-Tuned, pop music vocal-influenced form of music. Within pop music, the conflict over Auto-Tune is less pronounced: instead, the debate over the use of Auto-Tune with the genre pertains to whether a singer's use of Auto-Tune is in accordance with the high vocal aesthetic standard of pop music, one which places great emphasis on vocal and tonal precision, perfection, and high recording studio production values.

By exposing the underlying tuning and aesthetic preferences of both pop and hip hop, a discussion can be conducted regarding the various cultural and aesthetic values and implications which arise when a singer uses Auto-Tune, and to what degree the use of Auto-Tune either complements, challenges, or conflicts with the underlying aesthetic preferences of that artist's affiliated genre. In this thesis, I shall examine two primary musical genres, namely American pop music and American hip hop, and explore their respective musical values (i.e. what specific tuning preferences and expectations, musical sensibilities, aesthetic frameworks and principles the two genres espouse) in order to understand how 
the adoption of Auto-Tune has affected and/or challenged their respective musical conventions, aesthetics, practices, and values.

However, before such an examination can be conducted, it is necessary to first understand the technological underpinnings of Auto-Tune as a form of synthesis: as the product of a long lineage of analog and digital synthesizer development, and of various Digital Signal Processing protocols. Presenting this material is the purpose of Chapter II. In Chapter III, I shall explore the various methods by which Auto-Tune has been applied to vocal recordings within pop music, hip hop, and hip-pop (a hybrid subgenre of pop and hip hop), with the intention of establishing what impact the use of Auto-Tune has on the genres in which it is applied. In Chapter IV, I shall identify and explore precisely what aesthetic principles Auto-Tune either complements or conflicts with in both pop music and hip hop, with the intention of determining in what capacity genre aesthetics and conventions inform and/or influence Auto-Tune's reception. In Chapter V, I shall draw upon various theoretical models, such as Naomi Cumming's notions of expression and persona, Roland Barthes' grain of the voice, Jean Baudrillard's theory of hyperreality and simulation, and Jonathan Sterne's exploration of Truax and Schafer's notion of schizophonia, in order to examine what symbolic effect(s) Auto-Tune has upon the music it is applied to, as well as exploring how Auto-Tune can be understood in regards to these theoretical models. Finally, in Chapter VI, I will briefly highlight and explore the various ways Auto-Tune has extended from a musical post-production tool into a widely recognized pop culture trope, having appeared in television programs, 
been appropriated by internet users as a tool for satire and parody, spurred an increase in competing pitch-correction and vocal effect software and technology, and garnered noted reactions (and criticisms) from popular musicians in regards to pop culture's fascination with Auto-Tune. 


\section{II - Synthesis, Sampling, and Digital Signal Processing}

In order to fully understand the significance of Auto-Tune within modern popular music, it is necessary that we first explore the legacy of synthesizer (both analog and digital) and of Digital Signal Processing (DSP) technology. As an examination of early analog and digital synthesizer technology will show, the lineage of these technologies marks not only an evolution in technological capability, but the effect these technologies had upon the style, practice and performance of musicians themselves. This chapter will also explore synthesis as a technology -- one that contains within itself a distinction between analog and digital technology -- and as a concept which influences musical practice and sound. As well, the distinction between synthesis and resynthesis will be explored in order to explain how Auto-Tune practices and utilizes a particular form of synthesis. In order to fully understand the significance of Auto-Tune, we must also look back at the influence of recording technologies as a related practice, touching upon such topics as: Pro Tools, DSP techniques, and digital synthesis and sampling.

The underlying assumption of this chapter is that the incorporation of new musical (and recording) technologies impacts the practice, performance, and recording of music, sometimes leading to the formation of new subgenres and sometimes leading to conflict within existing genres. Not only will this chapter provide a historical pretext for understanding how Auto-Tune was created, but also provide a technical understanding of precisely what Auto-Tune is and how it 
has changed contemporary musical performance and recording practices. But first, in order to understand digital synthesis and DSP, we must explore the invention and influence of the Moog modular synthesizer, as well as the impact and influence of early synthesis in general.

\section{Moog Modular Synthesizer and Synthesis}

In October 1964, Robert Moog debuted a prototype at the American Engineering Society's annual convention: the world's first voltage-controlled, subtractive, modular synthesizer. Through its separate, component modules (e.g. oscillators, filters, envelope generators, etc.), the Moog synthesizer performed individual sound generation and modification tasks, which could be combined in various ways to create a singular, audible signal (synthesized together from the synthesizer's various components). These components were connected via patch cables, which allowed the synthesist to connect the signals generated through the various components and produce a unified, synthesized sound. The Moog modular synthesizer represented a significant milestone in that it was one of the first contemporary, mass-produced instruments to produce sound solely from circuitry instead of electrifying or amplifying an acoustic sound. The Moog Synthesizer was not merely a new musical instrument, it was an entirely new musical process: it generated new, unheard sounds through synthesis.

But what exactly is synthesis? According to Pinch and Trocco, "the classical meaning of 'to synthesize' is to assemble a whole out of parts. A 
synthesizer assembled parts of a sound into a complete sound. ${ }^{n 2}$ Robert Moog had created not only one of the few new musical instruments of the last century, but one that generated sound through the synthesis of various sonic components into a complete, unique sound. Not only was his instrument's sound a whole comprised of parts, but the parts themselves were produced through electronic circuits; he had not just electrified sound, his sound was electronic. This new ability to generate synthesized sound is one of many aspects which musicians found appealing about the synthesizer; it presented the musician with a vast horizon of creative possibility. A new "poetry-of-sound"3 arose out of the synthesizer, which helped to place the synthesist at the vanguard of modern musical instrument technology and sound. The same general principles applied to subsequent performance-oriented synthesizers such as the Minimoog or the EMS VCS 3, which enabled musicians to access this new horizon of sound (due in part to their lower cost). It also ushered in the era of the musician as synthesist, as someone not only versed in the technological composition of the device, but as competent enough to understand the function of the various components, and to creatively manipulate them in order to produce a new, unique sound.

The fundamental legacy of the synthesizer is that it marks the debut of the now common modern musical practice of isolating, modifying, and synthesizing

\footnotetext{
2 Trevor Pinch and Frank Trocco, Analog Days: The Invention and Impact of the Moog
} Synthesizer, (Harvard University Press, 2004), 67.

3 Pinch and Trocco, Analog Days, 274-275. 
sound waves themselves. What synthesis allows is the musician to generate a sound wave, and then potentially apply a huge range of options and possibilities in how the sound wave may be modified. This new musical practice and aesthetic, as well as the increasing materiality and malleability of music, would ultimately contribute to the foundational practices and production/editing techniques of the digital music era.

\section{Digital Synthesizers, Samplers and Processors}

While the Moog brand became widely synonymous with analog synthesizers, the next generation of synthesizers, which began to appear in the early-to-mid 1980s, made the transition to digital synthesis. With the advancement of digital computer technology in the late 1970s, manufacturers were beginning to mass-produce musical instruments capable of digitally recording, generating, and/or synthesizing sound. The first generation of instruments to harness this technological capability not only revamped the technical process of analog synthesis, but also established wide-reaching new digital music practices. For instance, New England Digital's Synclavier, released in 1975, was the first commercially available digital, sequencing synthesizer. It revolutionized the capability of commercially available synthesizers since it generated sound waves through digital circuitry and FM (Frequency Modulation) synthesis, where "one oscillator produce[s] a stable 'carrier' tone and another produce[s] a variable 'modulator' tone. This combination, when done correctly, could produce, with very little memory, sounds with rich palettes of frequencies 
and harmonics, ${ }^{n}$ but was entirely reliant on the precision of digital technology for effective implementation. As well, the Fairlight CMI (Computer Musical Instrument), the other major competitor to the Synclavier, introduced digital sampling to the broader (i.e., non-academic) music community in the early 1980s. This innovation inspired the designers at E-MU, who saw the potential that digital sampling presented, but quickly realized the Fairlight CMl's technical limitations: small amounts of memory, low sound resolution, and extremely high price point. The designers at E-MU responded by manufacturing a more effective digital sampler, which they called the Emulator. Publicly debuted in January 1981 , for the first time significant amounts of sound could be digitally sampled and played in the same manner as a synthesizer. Musicians could now capture external sounds and then play those sounds as if they were generated within the machine. This musical practice, of capturing and subsequently playing with recorded sound, would help form the backbone of how Auto-Tune is utilized and practiced within modern studio recording environments.

The impact of this new technological ability was instrumental in both the musical and aesthetic development of hip hop in the early 1980s. Sampling itself aesthetically complemented and roughly coincided with the slightly earlier technology and technique of turntablism, in which DJs would not only play snippets of sound from vinyl records, but would physically manipulate the records

\footnotetext{
${ }^{4}$ Greg Milner, Perfecting Sound Forever: An Aural History of Recorded Music, New York: Faber and Faber Inc., 2009), 310.
} 
themselves while spinning on the turntable, allowing the DJ to recontextualize the original recording into a new musical form. According to Joseph Schloss,

This technique would not have appealed to musicians from other genres, who wanted the freedom to create their own melodies and had no interest in digital recordings of other people's music. For those trained as hip-hop deejays, however, the ability to play an entire measure -- a break, in this case -- from an old record was exactly what they were looking for. ${ }^{5}$

Hip Hop producers quickly, and enthusiastically, endorsed and incorporated sampling into their production process, since it allowed them to sample preexisting sound and recontextualize it into their music. Again, Schloss highlights how sampling influenced the early aesthetic development of hip hop:

... because more than one loop could now be played simultaneously, producers could take their drums and their music from different records. With samplers, any music could be combined with a great drum pattern to make what is essentially a composite break ... This substantially broadened the spectrum of music that could be pressed into service for hip-hop. ${ }^{6}$

However, because the Emulator featured a limited amount of memory (RAM) -barely enough to record four bars of music -- many within the early hip hop production community were frustrated by these technological limitations, desiring "to sample entire passages from records, and repeat them to build a song. They didn't want just the sound of John Bonham's kick drum, they wanted to loop and repeat the whole 'When the Levee Breaks' intro."7 E-MU responded to this demand by releasing the SP-1200, their next-generation user-sampling drum

\footnotetext{
5 Joseph Glenn Schloss, Making Beats: The Art of Sample-Based Hip-Hop, (Middletown, CT: Wesleyan University Press, 2004), 35.

6 Schloss, Making Beats, 36.

7 Milner, Perfecting Sound Forever, 330.
} 
machine, which featured four times the RAM as their previous drum machine, but suffered -- according to the engineers at E-MU -- from the inclusion of a 12-bit sampling system (as opposed to the now standard 16-bit, CD-quality sampling rate), which ensured an inherent amount of distortion and loss of fidelity in the samples. Nevertheless, "the SP-1200 was a huge success. Hip Hop producers loved that it had enough memory for loops, and they loved its gritty sound."8 What the engineers at E-MU failed to recognize, and what many hip hop producers heard in the SP-1200, was that the device produced a sonic quality which resonated with hip hop's mid-to-late 1980s political ideology. As Greg Milner explains,

It's fitting that the hip-hop album that more than any other explored the intersection of politics and sonics, Public Enemy's It Takes A Nation of Millions to Hold Us Back, released in 1988, helped put the SP-1200 on the hip-hop map. The sound of that album was an explicit rejection of the traditional ideas of fidelity - both in and out of the hiphop world. The intricate abrasiveness of the music mirrored the pervasive lyrical theme of 'noise' - both as something politically desirable in an Attali/Adorno sense (Chuck D challenging black radio on "Bring the Noise") and as a perpetual miasma that hides the truth ("don't believe the hype $\left.{ }^{\prime \prime}\right) . .9$

With the E-MU SP-1200, many in the hip hop community found a voice for articulating and expressing their own political and aesthetic values, values which

... happened to dovetail perfectly with developments in hip hop during its unfolding golden age in the mid-and-late eighties. The music was moving away from a more traditional R\&Bderived sound and toward an aesthetic rooted in the concept of the DJ. Producers like Shocklee's Bomb Squad, Pete Rock,

\footnotetext{
8 Milner, Perfecting Sound Forever, 332.

9 Milner, Perfecting Sound Forever, 333.
} 
Marley Marl, and DJ Premier built tracks out of jagged, interlocking shards of sound, beats that 'slammed' as much as they swung, and musical motifs built on almost punishing repetition ... For them, the SP-1200's mechanistic feel was perfect. 'It quantized sound very abruptly,' Shocklee says. 'It was the thing that gave the SP-1200 its soul. ${ }^{10}$

For the first time, digital sound -- the abrupt and jagged sonic character of that sound (which will be explored further) -- found an aesthetic and thematic companion in hip hop.

With the introduction of these three new technologies in the early 1980s, three huge new categories were created in the field of music technology: the digital synthesizer, the digital processor, and the digital sampler. While these technologies share a number of commonalities, from the early 1980 s and onwards it is essential to distinguish synthesis and sampling as two separate (yet related) practices, even though (and perhaps especially because) the difference between them has become increasingly blurred through the practice of AutoTune. With digital synthesizers, all sound production occurs within the digital synthesizer's circuitry. With digital samplers, the original sound is external, recorded to the sampler's memory and then made available for various DSP editing effects (such as pitch shifting, filtering, phasing, etc.), which enables the producer to generate a recontextualized sound based upon the originally recorded sound. Not only could new sounds be synthesized and produced digitally, but digital samplers could potentially capture any perceivable sound and reconfigure that initial sound into a completely new sonic context. With the switch

\footnotetext{
10 Milner, Perfecting Sound Forever, 334.
} 
to digital synthesizers, samplers, processors, and recording consoles, musicians of all genres have been given an unprecedented ability to capture and reconstitute pre-existing sound, an ability which has fundamentally changed the way modern musicians create sound, as well as how they construct and compose songs. However, none of these innovations, whether produced through a digital synthesizer, sampler, processor, or recording console, would be possible without the innovation of digital recording and DSP technology.

\section{Digital Audio and Quantization}

As mentioned in the previous section, innovations in DSP technology in the late 1970 s and early 1980 s led to the production of more widely available digital synthesizers, samplers, processors and recording consoles, devices which dramatically influenced the sound, aesthetic and practice of various emerging and developing popular music genres such as hip hop. As well, with the growth and development of computer technology through the late 1970 s and 1980 s, computer hardware capabilities were expanding to accommodate ever-increasing and demanding computational processes. In particular, the growth in DSP was fueled by this increase in hardware capability: with increased processor speed, DSP algorithms could become more sophisticated and more widely utilized.

When an acoustic sound is sampled digitally, it undergoes a conversion

process known as Analog-to-Digital conversion. In this process, a continuous sound wave is sampled and digitized. In the conversion from analog sound wave to digital signal, the instantaneous amplitude of the analog sound wave is 
represented at regular time intervals by a digital value, and that string of recorded discrete values comes to digitally represent the sonic quality and character of the analog wave. During this quantization, the originally continuous sound wave is given a discrete, step-like representation. In essence, digital recording, sampling, and processing work by fitting the continuous values of a pressure wave onto a scale of discrete numerical values.

It is this very process which provides the fundamental, algorithmic basis of Auto-Tune: by quantizing the analog, acoustic sound wave, the digital representation produces discrete, continuous values. Once the values of the sound wave are digitized, it is then possible to modify or change particular values of the (digital) sound wave using any number of discrete algorithms: the values can be raised or lowered through DSP, which enables producers and/or engineers to subsequently change the pitch of the sound wave itself, along with many other possible transformations. Like all algorithmic operations on digitallyencoded information, what Auto-Tune allows is for these numerical values to be altered easily, thereby producing a change in the digital recording of a singer's voice.

With the switch to digital recording, sampling, and production, musicians, producers and engineers have been given newfound opportunities to experiment with editing, effecting, and altering their recordings. One of the strengths of digital representations of sound is that they can be processed with great flexibility and often non-destructively, which is not the case with analog signals. The quantized, 
digital sound wave can be modified in a variety of ways, since what is being altered is a digital representation of the analog sound wave, rather than the analog sound wave itself. This provides musicians, producers, engineers, etc., with an incredible ability to experiment with digital signals since any modifications made to the digital signal itself can easily be reversed and returned to its initial recorded state. With analog recording, however, it is painstakingly difficult to make significant edits and/or corrections to a performance when it has been recorded to magnetic tape or some other analog medium, and such edits are almost always destructive (irreversible). Digital recording and signal processing techniques eliminate many of these issues: effects can be tried out, and taken away, with no permanent repercussions. This represents a significant shift in not only the practice of recording music, but in what is feasibly possible in the realm of digital music.

\section{Pro-Tools}

With the advancements made in DSP technology, as well as in computer processing speeds and hard drive capacity, the transition to recording and mixing music on computer began to gain widespread momentum with the adoption of Pro-Tools, a software program that provides a graphic user interface which allows producers and engineers to record, edit, and mix music on computers. Initially developed by Peter Gotcher and Evan Brooks, the original version of ProTools debuted in 1984 as Sound Designer, a program designed to edit sound for the E-MU Emulator. In 1991, Digidesign debuted the first commercial version of Pro-Tools, but it was the 1997 version of Pro-Tools that sparked the great, 
industry-wide adoption of Pro-Tools: Pro-Tools 24 provided 24-bit audio (an extremely high audio resolution rate), 32 to 64 tracks, and the ability to record, edit, mix and master entirely on the computer and within the software program (referred to by producers and engineers as "in the box"). This sparked a widespread transition within the recording industry to recording through the ProTools interface, since it provides high resolution fidelity, a nearly infinite number of tracks, the ability to not only record and mix, but also edit the tracks being recorded/mixed, and the system costs significantly less than the multi-million dollar recording studios that dominated the industry up until that point. The migration from large, expensive studio consoles to small, relatively affordable, sometimes home-based recording studios was largely precipitated by the technical ability (and cost-effectiveness) of Pro-Tools.

For producers and engineers, Pro-Tools' greatest appeal is its ability to handle the recording and the mix within a graphical user interface that enables them to easily and infinitely edit, modify, customize and scrutinize a recording. Since Pro-Tools records sound digitally, it means the recording can be edited and modified in a variety of ways. Most significantly for the advent of Auto-Tune, ProTools accepts plugins: additional software programs that connect to Pro-Tools and allow for an expanded array of effects and modifications (such as dynamic range compression, pitch-correction, equalization, and/or audio effects such as reverb, delay, chorus, flanging, phasing, etc.). 
For musicians, Pro-Tools has fundamentally changed the way they record music. Instead of having to perform an entire take from beginning to end with absolute accuracy, musicians can now isolate specific, precise moments from multiple takes and combine them into a single, ideal take. However, this shift in musical practice from recording a performance in real time to using the studio to construct an ideal performance -- one that is assembled rather than performed -is not entirely a new one: such notions influenced The Beatles' retirement from the concert stage in order to explore the recording studio, as well as Glenn Gould's prophetic vision of even classical music recordings being a kind of idealization realized through the studio. These notions began to arise back in the 1960s with analog magnetic tape, but were hindered by the relative inalterability of magnetic tape itself. The ability and ease, however, of this vision to be practically realized is what distinguishes Pro-Tools.

One anecdote in particular helps to highlight this new recording aesthetic, and the effect it has upon modern recording artists. Greg Milner recounts an instance where British rock band The Kaiser Chiefs were asked to participate in a re-recording of The Beatles' "Getting Better" for the BBC's Abbey Road Sessions television show. ${ }^{11}$ Before the session began, the producers decided to record the session in the same manner in which The Beatles' recorded the song for their 1967 masterpiece, Sgt. Pepper's Lonely Hearts Club Band: on 4-track analog tape. This decision greatly affected the productivity of the session:

\footnotetext{
11 "Kaiser Chiefs - Getting Better (Beatles Cover)," YouTube video, 2:49, from a performance televised on BBC Radio 2 on June 2, 2007, uploaded by tommarques on June 3, 2007, accessed February 16, 2012, http://www youtube,com/watch? $v=n C c U 2 x f v z 00$.
} 
As [The Kaiser Chiefs] play the basic tracks, you can see [Geoff Emerick, the Beatles' recording engineer] wearing a perplexed look in the control room. Something isn't quite right. The band is competent, but they're not really nailing it. "No," Emerick says, and calls them into the control room. They look sheepish. They ask the cameras to be shut off and spend two hours conferring. They manage to get through the song to Emerick's satisfaction, and afterwards reflect on the experience. ${ }^{12}$

The reason the band was not "nailing it" was because the band, like many modern rock bands, was not used to the limitations of 4-track analog tape: not only were overdubs kept to a minimum, but the format itself forced the musicians to provide perfect performances. This, for a band whose primary experience with recording has been the Pro-Tools variety, was a complete shock. The band's lead singer, Ricky Wilson, subsequently expressed a new-found appreciation for the older format and recording process: "It sounds more lively and it sounds more live, and it doesn't sound like instruments playing separately ... I can actually hear the difference, which I didn't think l'd be able to do." ${ }^{13}$ With the considerable technological advancements that recording studio technology has made over the last 50 years, from analog tape to nearly infinite multitrack digital recording, the most significant change in recording studio practice and culture has been to musicians: with Digital Audio Workstations like Pro-Tools, modern musicians no longer have to perform or record in the same manner as their musical predecessors. These highly sophisticated programs allow producers and engineers to cut and paste together an ideal performance, to create a perfect take, rather than capture it. This process indeed makes the recording studio

\footnotetext{
12 Milner, Perfecting Sound Forever, 300.

${ }^{13}$ Greg Milner, Perfecting Sound Forever, 300.
} 
more productive, and it provides a means for realizing an ideal recording, but it also de-emphasizes a number of musical skills. Ultimately, as the Kaiser Chiefs' experience with 4-track analog recording demonstrates, this is a catch-22: while the modern recording studio allows musicians, producers, and engineers to realize their ideal recording, it conversely fosters a recording studio work ethic of reliance upon post production editing techniques. A similar work ethic is arising in regards to Auto-Tune: instead of singers being required or expected to sing precisely and accurately in a single (or several) takes, mistakes and imperfections are permitted to be recorded, on the assumption that the producer or engineer will correct the performance in post-production using Auto-Tune.

\section{Auto-Tune}

And it is from here, the realm of Pro-Tools plugins, where the history and origin of Auto-Tune can be directly addressed and explored. Andy Hildebrand, the creator of Auto-Tune, was initially an engineer for Exxon Mobile, spending 18 years specializing in the interpreting of seismic data while in search of oil. The process of "drilling for oil involves setting off dynamite charges, recording the way the sound reflects off the subsurface layers of the Earth, and producing an image of those layers that provides clues as to the possible existence of oil. ${ }^{n 14}$ In essence, Hildebrand was honing his skills and expertise in the DSP subfield of seismic data. Hildebrand eventually left the oil industry to study musical composition at Rice University, and soon wrote a looping program called Infinity

\footnotetext{
14 Milner, Perfecting Sound Forever, 342.
} 
which addressed the problem of looping a collection of sounds to form a complex waveform. According to Milner,

At an AES convention in 1996 or 1997, Hildebrand was speaking with the wife of one of his distributors. She was a choral singer and mentioned that it would be great to have a device that would make her sing in tune. Hildebrand realized that the same type of processing that he had used in the oil industry could be put to work as a pitch-corrector. A year later, at a trade show, Hildebrand showed off the first version of Auto-Tune... ${ }^{15}$

Whether Milner's account is entirely accurate or not, the fact remains that Hildebrand came to the realization that the same DSP techniques used in seismic data interpretation could be adapted to quantize and alter the pitch of a singer, bringing them back in-tune. Auto-Tune was a very big hit at the 1996 AES convention, and Hildebrand eventually signed a licensing agreement with Antares Audio Technologies, who have since developed and distributed the program to great success.

However, Auto-Tune does not represent the first iteration of vocal effect technology. For instance: the Vocoder was originally developed in the 1930 s by Homer Dudley at Bell Laboratories as a signal processing technology for the military to encode, encrypt, compress, and transmit speech "in an attempt to save early telephone circuit bandwidth. ${ }^{16}$ By the 1970 s, the Vocoder began to be adopted within popular music as a way of generating uniquely synthesized

\footnotetext{
${ }^{15}$ Milner, Perfecting Sound Forever, 342.

16 Wendy Carlos, "Postscript Notes On Vocoders," WendyCarlos.com, accessed February 16, 2012, http://www. wendycarlos.com/vocoders.html.
} 
vocals, as can be heard in Pink Floyd's "Sheep,"17 Aerosmith's "Sweet Emotion, ${ }^{18}$ and for a large part of Neil Young's 1982 album Trans. ${ }^{19}$ While AutoTune may have found its inception in connection to seismic data interpretation, whereas the Vocoder was intended to compress speech in order to conserve telecommunication bandwidth and to aid encryption, both technologies were eventually introduced into musical practice and subsequently became technologies used to produce -- through resynthesis -- unique vocal effects.

Whereas digital synthesizers and samplers primarily play back sonic information without (necessarily) fusing it with anything else, Auto-Tune is, in essence, a resynthesizer: like the Vocoder, which imposes a spectral analysis upon an outside source (thereby producing a unique vocal effect), Auto-Tune imposes a quantized DSP analysis upon an external vocal source. Auto-Tune essentially blurs the distinction between sampling and synthesis by sampling (through digital recording consoles) a singer's voice, performing various DSP modifications and corrections to the digital signal, and fusing those modifications back into the digitally recorded vocals. Auto-Tune, therefore, can be considered a modern, digital successor of earlier vocal processing and resynthesis effect technology (a la the Vocoder). As well, technically speaking,

What Auto-tune could do was not entirely new. Very skilled engineers could correct pitch with various methods,

\footnotetext{
17 Pink Floyd, "Sheep," Animals, EMI Music Canada B004ZN9UZO, 2011, compact disc, 2011, originally released in 1977 .

${ }^{18}$ Aerosmith, "Sweet Emotion," Toys in the Attic, Sony BMG Music Entertainment B0000029AP, compact disc, 1994, originally released in 1975.

19 Neil Young, Trans, Geffen B000024R1I, compact disc, 1995, originally released in 1982.
} 
including sampling the voice and manually manipulating a sort of pitch wheel. But as with other facets of the ProTooled world, Auto-Tune made a formerly difficult task so easy anybody could do it. You tell the program what scale and key to use as a reference point, and it continually samples the singer's voice and decides which of the correct notes is closest, adjusting accordingly. ${ }^{20}$

The ease with which engineers and producers can pitch-correct a recorded vocal demonstrates Auto-Tune's single greatest appeal: like Pro-Tools itself, Hildebrand's software made formerly laborious and time consuming recording studio practices quick, easy, accurate and cost-effective. And since Auto-Tune could plug in to Pro-Tools, the adoption of this software was not only effortless, but widespread. With Auto-Tune, the technologies of sampling, synthesis, and resynthesis not only combine in practice but blur together into a new, postproduction correction aesthetic. (This process of resynthesis will be considered further in Chapter $V$ to help explore Jean Baudrillard's notions of hyperreality and simulation.)

However, what Hildebrand himself did not seem to have predicted was how his pitch-correction software could also be used as a special effect:

Although Auto-Tune was designed as a corrective device, it broke into the mainstream as a creative sound processor. While working with Cher on the song "Believe" in 1998, producers Mark Taylor and Brian Rawling discovered that if they set Auto-Tune on its most aggressive setting, so that it corrected the pitch at the exact moment it received the signal, the result was an unsettling robotic tone. When "Believe" became a huge worldwide hit, Taylor and Rawling initially tried to keep their Auto-Tune trick a secret, although

20 Milner, Perfecting Sound Forever, 342. 
word soon got out and the 'Cher effect' became a ubiquitous production tool over the next few years. ${ }^{21}$

Through Taylor and Rawling's creativity and experimentation with Auto-Tune's various settings, the duo discovered that the program could be used to generate a unique vocal effect in addition to a pitch-correction. The discovery of the Chereffect recast Auto-Tune as both a pitch-correction editing software program and a vocal effect signal processor akin to the vocoder in its general sound. Hildebrand's original software has also grown to become quite versatile in more recent versions: Auto-Tune 7 (the most recent edition) not only allows the user to "instantaneously detect the pitch of the input, [identify] the closest pitch in a userspecified scale (including minor, major, chromatic and 26 historical and microtonal scales), and [correct] the input pitch to match the scale pitch, ${ }^{22}$ but also provides a new time correction and manipulation system, which allows producers and/or engineers "to quickly and easily edit the timing of your vocals right along with their pitch. ${ }^{23}$ Antares Audio Technologies have also expanded Auto-Tune into a platform for which a number of spin-off vocal processing plugins have been developed, known as the AVOX EVO Tool Kit, which further augment and increase the software's vocal effect capabilities.

What Antares' suite of vocal processing plugins demonstrates is that AutoTune's pitch-correction technology and abilities are only the beginning: with the

\footnotetext{
${ }^{21}$ Milner, Perfecting Sound Forever, 342-343.

22 "Antares Auto-Tune 7 Pitch and Time Correcting Plug-In," Antares Audio Technologies, accessed February 16, 2012, htto://umw.antarestech.com/products/auto-tune-7.shtml.

23 "Antares Auto-Tune 7 Pitch and Time Correcting Plug-In," Antares Audio Technologies, accessed February 16, 2012, http://www.antarestech.com/products/auto-tune-7.shtml.
} 
advances made in computer processing power and DSP protocols, producers and engineers now have the computing ability to effect, edit, and refine vocals in ways both easily achievable and only recently possible. For the moment, AutoTune remains the most appealing and widely used vocal pitch-correction software because it provides a seamless integration with Pro-Tools, presents a clean and user-friendly interface, and makes the task of vocal pitch-correction fast, easy, convenient, and very cost-effective.

\section{Conclusion}

But how has Auto-Tune, like the analog and digital synthesizer, digital sampler, and Pro-Tools before it, affected the protocols and conventions of musical performance and/or recording practices? The answer can be best understood in three parts: first, in terms of studio recording practices, musicians and producers across many genres are increasingly turning to Auto-Tune for a variety of reasons, whether it be to "touch-up" a flat or sharp vocal note in an otherwise acceptable recording/performance (in accordance with whatever performative or stylistic standard or expectation that musician aspires to), or whether it is to experiment with the Cher-effect within their own music. In much the same way that Pro-Tools has fostered a new work ethic amongst musicians, producers and engineers, Auto-Tune has similarly fostered a new recording studio work ethic amongst singers and vocalists. Auto-Tune enables a new approach to recording vocals, one that de-emphasizes the technique and skill of recording live off the floor, in favour of a post production correction approach. 
Second, the use of Auto-Tune within live musical performance is increasing. While Auto-Tune is predominantly used within the recording studio, the software has proven to be agile enough to handle real-time, live performance pitch correction, and has thus become a tempting safety net for live performers. For instance, it has been reported that country music singers such as Reba Mclntyre, Faith Hill and Tim McGraw have all used Auto-Tune in their live performances as a safety net against either wrong or stray notes, ${ }^{24}$ while pop musicians (such as Cher, Britney Spears, Lady Gaga, and others) have also used Auto-Tune in their live performances for both its corrective and effective attributes. The work ethic fostered by Pro-Tools and Auto-Tune within the modern recording studio is beginning to extend to the concert stage: artists are beginning to rely upon Auto-Tune for not only their studio work, but their live performances as well.

Finally, the increased usage of Auto-Tune, for both its clandestine pitch correction and its more overt Cher-effect, has led to changes in the genres in which it is most commonly used. Within pop music, Auto-Tune has become a valued, desired sound effect: artists from across the genre are incorporating Auto-Tune into their recordings in a number of unique ways, some for its pitch correction capability, some for its unique vocal effect. In either scenario, the use of Auto-Tune has become highly valued within pop music, since it represents the

${ }^{24}$ Christopher John Tracy, "Pitch-adjusting software brings studio tricks," Boston Herald, February 19, 2007, accessed February 16, 2012, http://pqasb.pqarchiver.com/bostonherald/access/ 1218937871 $\mathrm{htm}$ ? dids =1218937871:12189378718FMT=ABS\&FMTS=ABS:FT \&date=Feb $\pm 19 \% 2 \mathrm{C}+2007$ \&author $=\mathrm{CHRISTOPHER}+\mathrm{JOHN}+\mathrm{TREACY}$ \&pub=Boston + Herald \&edition $=\&$ startpage $=32 \&$ desc $=$ MUS $\mid \mathrm{C}+\% 3 \mathrm{~B}+$ Pitch-adjusting + software + brings + studio tricks. 
new vanguard of modern pop music (both sonically and aesthetically), and it upholds pop music's vocal aesthetics of precision and perfection. In hip hop, on the other hand, the import of Auto-Tune within the genre has become highly contentious: a considerable amount of internal discourse and strife has arisen out of the use of Auto-Tune within hip hop, between a cadre of young, new artists who have incorporated the technology into their music, and an older, established sect of artists who cling to an old-school aesthetic of hip hop which is at odds with Auto-Tune. In Chapter IV, I shall venture to explain precisely why the utilization of Auto-Tune within pop music and hip hop has sparked such distinct reactions by exploring the aesthetic and cultural values of each respective genre, and to what degree Auto-Tune either complements or conflicts with those values.

However, before such an analysis can be adequately conducted, I shall first explore -- in Chapter III -- the methods and techniques in which Auto-Tune is applied and utilized within a number of musical examples. Drawing upon pop music, hip hop, and "hip-pop" -- a hip hop subgenre which has experienced a recent resurgence through hip hop's experimentations with Auto-Tune -- I shall endeavour to highlight Auto-Tune's effect upon the music it is applied to. 


\section{III - Auto-Tune Within Popular Music}

As alluded to in the conclusion to the previous chapter, Chapter III will contain examinations of precisely how Auto-Tune is implemented and utilized by a variety of artists, how the use of Auto-Tune affects the musician's particular musical idiom, as well as a general overview of the larger trends and methods by which Auto-Tune is incorporated into the body of pop music and hip hop. Finally, Chapter III will include a brief examination of whether the artist's use of AutoTune complements or conflicts with the tuning preferences and aesthetic values of their respective genre, which will play a much larger role throughout Chapter IV.

While pop music has, generally speaking, more easily and successfully adopted and incorporated Auto-Tune (both as clandestine pitch correction and as overt effect), the use of Auto-Tune within hip hop has led to the development of two key conditions: first, a contentious discourse and debate within the hip hop community over the use of Auto-Tune within their genre, and second the resurgence of the hip hop subgenre referred to as hip-pop (a crossover between hip hop and pop music). While this term has come into (relatively) common

usage over the last several years, its use predates the import of Auto-Tune into hip hop, suggesting that while Auto-Tune is indeed relevant to the genre, it is not necessarily the cause of this stylistic fusion. 


\section{Pop Music}

Pop music was one of the first genres to begin experimenting with AutoTune in the mainstream record industry. Auto-Tune's use within the industry in the first few years of its availability was modest, considered by engineers and/or producers as a trade secret. Few producers or musicians initially discussed the program, nor did they wish to divulge to which artists the technology had been used to touch-up their vocal recording(s). For instance, producers Mark Taylor and Brian Rawling, while working on Cher's 1998 hit single "Believe,"25 even tried to thwart their colleagues by claiming they had discovered the distinctive AutoTune vocal effect through a creative use of the Digitech Talker Vocoder pedal, rather than through the then little-known Antares Auto-Tune plugin. ${ }^{26}$ It was not long, however, until other producers and engineers began to decipher Taylor and Rawling's technique, and to apply the Auto-Tune effect within their own projects.

Nevertheless, Auto-Tune was initially used by producers and engineers along precisely the parameters which Andy Hildebrand had originally envisioned and designed: as a clandestine, post production pitch-correction technology. This technique is still widely applied within popular music, although it is less evident and/or overt in its other usage, the robotic, flutter vocal effect. However, a number of instances exist on record where traces of the Auto-Tune pitch

${ }^{25}$ Cher, "Believe," Believe, Wamer Brothers B00002430K, compact disc, 1998.

26 Sue Sillitoe, "Recording Cher's Believe," Sound on Sound, February 2009, accessed February 16, 2012, http://www.soundonsound.com/sos/feb99/articles/tracks661.htm. 
correction are evident in a vocal performance. For example, in Maroon 5's "She Will Be Loved," ${ }^{27}$ singer Adam Levine's delivery of the words "everyday," "rain," "smile," and "awhile" in the chorus bear the residual trace of an Auto-Tune touchup: the two notes briefly jolt, then lock into a flat, stable tone. This is evidence of the use of Auto-Tune because normally a singer's voice, however pitch-perfect they may be, will inevitably waver. When a singer's voice produces a note that does not waver, it is likely that Auto-Tune has been applied to either correct or stabilize the note in question. The same principle applies in the case of Katy Perry's "Last Friday Night (T.G.I.F.), ${ }^{28}$ where during the pre-chorus, Perry's vocals are Auto-Tuned in order to produce clear, stabilized tones. This effect is most evident in the lyrics "of last night" and "ended up online," where Perry's vocal delivery, while in-tune, also contains no vocal wavering, and is more akin to a drone. Another example, also from Katy Perry, is in her song "California Gurls (feat. Snoop Dogg), ${ }^{29}$ where during the chorus, Perry performs an octave jump between two notes. The use of Auto-Tune is evident in two ways: first, instead of a clear octave jump between two notes, Perry's delivery sounds more like an ascending and descending slide between the two octaves (a sonic by-product of Auto-Tune's rapid pitch correction). Second, the notes Perry hits in that octave jump are themselves pitch-perfect, a difficult task to accomplish for a vocalist

\footnotetext{
27 Maroon 5, "She Will Be Loved," Songs About Jane, Universal Music Group B00006879E, compact disc, 2002.

${ }^{28}$ Katy Perry, "Last Friday Night (T.G.I.F.)," Teenage Dream, Capitol Records LLC B003L77TZI, compact disc, 2010.

29 Katy Perry, "California Gurls (feat. Snoop Dogg)" Teenage Dream, Capitol Records LLC B003L77TZI, compact disc, 2010.
} 
without the aid of Auto-Tune. This is evident in Perry's live performance of the song on The Late Show with David Letterman, ${ }^{30}$ in which she performs the octave jump (at 2:26) in a less than precise, clearly unassisted, yet entirely realistic manner. Therefore, given the high technical precision of the final studio recording, it is quite likely that Perry's delivery was Auto-Tuned in order to render a pitch-perfect octave jump.

There are other ways in which Auto-Tune's residual trace can be detected: for example, in Avril Lavigne's breakout single "Complicated,"31 the use of AutoTune is evident in her delivery of the words "way," "when," "driving," "you're" and "become" during the song's second verse. In this instance, instead of producing a clear, stabilized, pitch-perfect tone, the application of Auto-Tune is detectable in the brief, rapid flutter between notes, akin to a very rapid tremolo. In this instance, the program is rapidly pitch correcting Lavigne's delivery, which contains a number of stray, intentionally off-key notes, into a rigorously in-tune pitch. This is an interesting example: since Lavigne is intentionally singing in a loose manner, flirting with notes that are off-key, it is most likely Lavigne's vocals were Auto-Tuned in order to maintain a stylistic adherence to a pop music aesthetic of pitch-perfect vocal tuning. A similar manifestation of this vocal aesthetic can be found in the songs of pop music singer Ke\$ha: in songs such as

\footnotetext{
30 "Katy Perry - California Gurls (Live On Letterman), YouTube video, 3:50, from a performance on The Late Show with David Letterman, uploaded by KatyPerryVEVO on October 18, 2010, accessed February 16, 2012, http://wwww.youtube.com/watch? $y=j u s E L 6 d r c h E \&$ feature $=m f u$ in order\&list $=U L$.

31 Avril Lavigne, "Complicated," Let Go, Sony Music Canada Inc. B000066NWO, compact disc, 2002.
} 



vocal delivery, particularly in her verses. In this instance, it is most likely that the application of Auto-Tune is less about pitch correction than it is about pitch control: the program allows Ke-\$ha to bend her notes without over stepping the boundaries of pitch and/or going out of tune. The application of Auto-Tune permits Ke\$ha to sing in a slightly off-key, drawled vocal style, while still maintaining a precisely in-tune vocal pitch. However, the residual traces of AutoTune's use still remain: in the pre-chorus to "We $R$ Who We $R$," the words "glitter," "eyes," "ripped," and "sides" contain a rapid vocal flutter, evidence of an Auto-Tune pitch correction having been applied to Ke\$ha's vocal performance.

Other examples of the rapid Auto-Tune pitch correction vocal flutter within pop music include Natasha Bedingfield's "Love Like This (feat. Sean Kingston), ${ }^{\text {"34 }}$ particularly in the verse lyrics "That's why you keep on runnin' / in and out of my mind," and in the chorus lyrics "Well this life tries to keep us apart / you keep callin' me back to your heart," which demonstrates the manner in which Auto-Tune rapidly pitch-corrects a vocal flourish. Rebecca Black's "Friday, ${ }^{35}$ a viral YouTube video which featured the aspiring 14-year old pop star's debut single, features copious amounts of Auto-Tune. Auto-Tune is applied to Black's

32 Ke\$ha, "Tik Tok," Animal, Sony Music Canada Inc. B002XNEll2, compact disc, 2009.

${ }^{33}$ Ke\$ha, "We R Who We R," Cannibal, RCA Records/Sony Music Entertainment B0046M14RG, compact disc, 2010.

${ }^{34}$ Natasha Bedingfield, "Love Like This (feat. Sean Kingston)," Pocketful of Sunshine, Sony Music Canada Inc. B000Y14U4M, compact disc, 2008.

35 "Rebecca Black - Friday - Official Music Video," YouTube video, 3:48, uploaded by rebecca on September 16, 2011, accessed February 16, 2012, http://www.youtube.com/watch? $\mathrm{v}=\mathrm{kfVsfOSb}, \mathrm{YOQ}$ \&list=FLa6BeLNHFOzz13sMOOPNu-g\&index $=6$. 
vocals throughout the song: in the verses, the program is used to produce a flat, unwavering tone to her vocals, while in the second verse the program is clearly applied to her vocal flourishes on lyrics such as "I got this, you got this," and "now you know it." Even more so, Auto-Tune is also applied to Black's background vocals, particularly in the song's intro and final chorus. Black's "Friday," produced by the Los Angeles based Ark Music Factory (a music production company which specializes in producing teen-oriented pop music for aspiring female teenage singers), is indicative of a particular set of vocal aesthetics expected by contemporary pop music: a highly processed and/or pitch corrected vocal performance, in keeping with the genre's stylistic expectations of precision and perfect vocal pitch.

While pitch correction through Auto-Tune has become an increasingly common practice amongst recording studios, producers and engineers in the last decade, so has Auto-Tune's other usage: as an overt vocal effect. As mentioned previously, producers Mark Taylor and Brian Rawling, while working on Cher's 1998 hit single "Believe," essentially discovered through experimentation that the pitch correction software could be used to produce a unique, robotic vocal effect. Taylor and Rawling discovered that Auto-Tune's settings enabled the ability to produce a unique vocal effect "when the pitch correction speed is set too fast for the audio that it is processing. ${ }^{36}$ This produced a rapid flutter in the singer's vocals, sonically akin to a digital glitch. This digital glitch/flutter vocal effect fit within the zeitgeist of the late 1990's, which saw the increasing digitization of

${ }^{36}$ Sue Sillitoe, “Recording Cher's Believe.” 
modern culture as well as the increased popularity and influence of electronic music in both pop music and rock/alternative music. (U2's "Discotheque"37 and The Prodigy's "Breathe" 38 are two songs which demonstrate the increased experimentation with electronic music and rock/alternative music that occurred in the late 1990s, while Jennifer Lopez's "Waiting For Tonight"39 and Cher's "Believe" provide examples of electronic music's influence on pop music.) The effect was not only a huge hit with Cher herself (who ultimately approved its use in the final recording), but also hugely successful commercially for Cher: since the song's debut in $1998,375,000$ digital and 1.8 million physical singles of the song "Believe," as well as 3.6 million physical copies of the album Believe, have been sold to date.40 The song's production also greatly influenced and inspired fellow musicians, producers, and engineers to not only decipher how the effect was created, but apply it to their own respective projects. It even led to the coining of the informal term Cher-effect, "used to describe extreme digital pitch correction of singing to produce a semi-artificial voice sound effect. ${ }^{n 1}$

While Cher's "Believe" applied the Auto-Tune vocal effect to particular vocal flourishes throughout the song, other electronic music acts such as Eiffel

37 U2, "Discoteque," Pop, Universal Music Group B000001EAQ, compact disc, 1997.

38 The Prodigy, "Breathe," Fat of the Land, XL Recordings B000002NFM, compact disc, 1997.

39 Jennifer Lopez, "Waiting For Tonight," On the 6, Sony Music Canada Inc. B00000J7RZ, compact disc, 1999.

40 Gary Trust, "Ask Billboard: Twice Is Nice, Twice, for Adele," Billboard.com, November 5, 2011, accessed March 28, 2012, http://wwwwbillboard,com/\#/column/chartbeat/ask-billboard-twice-isnice-twice-for-adele-1005477572.story?page $=2$.

41 "Cher-Effect," WikiAudio.org, accessed February 16, 2012, http://en.wikiaudio.org/Cher effect. 
65 were beginning to apply the Auto-Tune effect to an entire vocal performance, as can be heard in their song "Blue (Da Ba Dee). ${ }^{42}$ While Eiffel 65 's song became somewhat of a novelty dance/electronic music hit, Daft Punk's 2001 hit single "One More Time, ${ }^{\text {n3 }}$ much like Cher's "Believe," was a crossover hit, becoming commercially successful on the Billboard dance/club, pop, and Hot 100 music charts. ${ }^{44}$ While Cher's "Believe" utilized the Auto-Tune effect in certain places, namely towards expressive phrases in the verses (the lyrics "can't do that,") and the chorus ("I can feel something inside myself"), Eiffel 65 and Daft Punk instead applied the Auto-Tune effect to the entire vocal performance. The manner in which Auto-Tune is utilized in "One More Time" is methodologically similar to how the Vocoder is used in Daft Punk's 1997 hit single "Around The World, ${ }^{45}$ in which the entire vocal performance is heavily processed. This approach recast the Auto-Tune vocal effect from a momentary accent used to emphasize the lyrics to a consistent sonic effect that complemented the electronic instrumentation that underlay the song. By applying the Auto-Tune effect to the entire vocal performance, Eiffel 65 and Daft Punk effectively recontextualize the Auto-Tune vocal effect into a vocal sound; as a unique vocal character and/or persona which the singer could assume (a concept of Naomi

\footnotetext{
42 Eiffel 65, "Blue (Da Ba Dee)," Europop, Bliss Co. B00003GPOT, compact disc, 1999.

43 Daft Punk, "One More Time," Discovery, EMI Music Canada B000059MEK, compact disc, 2001.

44 "One More Time - Daft Punk I Billboard.com," Billboard.com, accessed March 28, 2012, http:// www.billboard.com/artist/daft-punk/chart-history/141900\#/song/daft-punk/one-more-time/ 3072399.

45 Daft Punk, "Around The World," Homework, EMI Music Canada B000000WCV, compact disc, 1997.
} 
Cumming's which will be explored in Chapter V). As well, "Blue (Da Ba Dee)" and "One More Time" essentially establish a template for how Auto-Tune can be applied to a lead pop vocal performance backed by electronic instrumentation. More importantly, given the increased crossover and influence between electronic music and pop music in the late 1990s, this template could be applied to both electronic music and to pop music vocal performances.

In the subsequent years since the discovery of Auto-Tune's distinctive vocal effect, other pop music artists have experimented with and/or incorporated the Auto-Tune Cher-effect into their music. In 2000, Madonna applied the AutoTune effect (as well as an array of other vocal DSP effects) to her performance in the song "Impressive Instant."46 Britney Spears' 2003 single "Toxic" 47 incorporates a sizable amount of both Auto-Tune (in Spears' delivery of the verses) and a multiplicity of DSP effects (particularly in the song's bridge, where Spears' voice is sampled, fragmented, and manipulated in a multitude of ways, culminating in a highly synthesized vocal effect applied to the bridge's final lyrics "With the taste of your lips / I'm on a ride"). Rihanna used the effect in her 2008 single "Disturbia," 48 while Lady Gaga applied the effect throughout her 2009 song "Monster," ${ }^{\text {"9 }}$ particularly in the song's bridge. Lady Gaga's use of Auto-Tune is intriguing, considering she has demonstrated and discussed publicly (on Ellen

\footnotetext{
46 Madonna, "Impressive Instant," Music, Warner Bros. Music B00004X01U, compact disc, 2000.

${ }^{47}$ Britney Spears, "Toxic," In the Zone, Sony Music Canada B0000DD7LB, compact disc, 2003.

48 Rihanna, "Disturbia," Good Girl Gone Bad, The Island/Def Jam Music Group B0018S6YO4, compact disc, 2008.

49 Lady Gaga, "Monster," The Fame Monster, Interscope B002W6ZOUK, compact disc, 2009.
} 
Degeneres' talk-show Ellen ${ }^{50}$ that she can indeed sing. Her usage of Auto-Tune within her music is therefore stylistic: she applies the Cher-effect in the song, not to correct any off-key pitches, but to experiment with the effect, to play with the vocal character that the effect produces.

What the use of Auto-Tune amongst all these artists demonstrates is pop music's openness to experimentation with new digital technologies and cutting edge, vanguard electronic music techniques and practices. As well, it also demonstrates pop music's aesthetic preference and tendency for what Serge Lacasse calls vocal staging, which

refers to any deliberate practice whose aim is to enhance a vocal sound, alter its timbre, or present it in a given spatial and/or temporal configuration with the help of any mechanical or electrical process, presumably in order to produce some effect on potential or actual listeners. ${ }^{51}$

The application of Auto-Tune to a pop singer's recorded vocal performance indicates not only a general predilection in pop music towards the vocal staging of a singer's voice, but also the very particular vocal setting -- which Lacasse defines as "a specific configuration of vocal staging whose characteristics are described in terms of loudness, timbral quality, and spatial and temporal

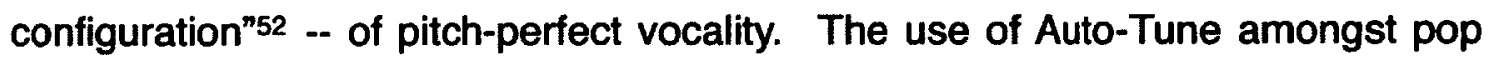
music vocalists, according to Lacasse, would suggest that the use of such pitch

\footnotetext{
50 "Lady Gaga Interview, Ellen DeGeneres Show 11/27/2009," YouTube Video, 7:39, uploaded by Igvideos 176 on April 7, 2011, accessed February 16, 2012, http://youtu.be/q18QZiaA4Bg.

51 Serge Lacasse, “'Listen to My Voice': The Evocative Power of Vocal Staging in Recorded Rock Music and Other Forms of Vocal Expression" (PhD diss., University of Liverpool, 2000), 13.

52 Lacasse, "Listen to My Voice," 14.
} 
correction and vocal effect technology is applied in order to achieve a very particular (and desired) vocal setting, one which could potentially 'produce some effect on potential or actual listeners' and 'give rise to a range of connotations and effects whose 'emergence' in the listener's mind is not arbitrary, but rather coherent. ${ }^{n 3}$

\section{Hip Hop}

While pop music has enthusiastically experimented with, adopted, and applied Auto-Tune to many recorded vocal performances, the adoption and application of Auto-Tune within hip hop has been more contentious. Currently, within contemporary hip hop, the attitudes and reactions amongst musicians and listeners towards the use of Auto-Tune are not only mixed but polarizing. ${ }^{54}$ AutoTune's import into hip hop has caused a schism within the genre, deeply dividing these artists into two primary camps: established hip hop artists who resist and reject Auto-Tune in the name of an old-school aesthetic, against a younger generation of Auto-Tune proponents. Why these two groups fundamentally disagree over the use of Auto-Tune within the genre of hip hop will be explored in more detail in Chapter IV. For now, it is necessary to examine the ways in which Auto-Tune has been applied within hip hop, in order to later understand their reasons and/or reactions.

\footnotetext{
53 Lacasse, "Listen to My Voice," 3.

54 NWSO, "BLOG: I Heart Kanye West (A Preview of Sorts)," XXLmag.com, October 28, 2008, accessed February 16, 2012, http://www.xxlmag.com/bloggers/2008/10/i-heart-kanye-west-apreview-of-sorts/comment-page-2/.
} 
By the early 2000 s the old-school hip hop paradigm itself was beginning to undergo fundamental changes which would lead to the fostering of an environment in which the experimentation with, and emergence of Auto-Tune in hip hop would not only occur, but would achieve both artistic and commercial success. Geographical shifts were beginning to break down the east-coast vs. west-coast paradigm, particularly with the rise of independent hip hop scenes and communities in St. Louis (with artists such as Nelly), Chicago (Common, Kanye West), Detroit (Eminem, Slum Village) and the collective of southern United States cities (such as Atlanta, Nashville, Memphis, New Orleans, Orlando, Miami, etc.,) which have come to be referred to as "the Dirty South." The Dirty South's sound can be broadly understood and referred to as crunk, a subgenre of hip hop that developed in Memphis with artist Lil' Jon, but which has also come to be used as a blanket term for all southern hip hop music artists and scenes. Crunk's unique brand of programmed, minimalist, drum machine rhythms, deep subwoofer bass lines, synthesizers and call-and-response MC declarations (rather than lyrics) stood apart from the conventional hip hop instrumentation and production practices of old-school, east-coast vs. west-coast hip hop (which instrumentally relied upon continuously repeating samples, produced and manipulated by the DJ on turntables or with a sampler). More importantly, crunk's mainstream success -- which occurred circa 2003 with Lil Jon \& The East Side Boyz' "Get Low," 55 and "Yeah!" by Usher6 .- as well as the subsequent rise of the

\footnotetext{
55 Lil Jon \& The East Side Boyz, "Get Low," Kings of Crunk, Universal Music Group B00006GA4S, compact disc, 2002.

56 Usher, "Yeah!," Confessions, BMG/Zomba Recording LLC B0002ZYDKQ, compact disc, 2004.
} 
Dirty South amongst the broader hip hop community represented a new musical aesthetic, independent of and distinct from the old-school hip hop tradition. The mainstream success of the Dirty South's new musical aesthetic helped in-turn to foster and encourage a community of producers and MCs to begin exploring and experimenting with new sounds and musical practices in the pursuit of establishing and/or further developing their own unique sound.

In much the same way that Cher is credited as being the first artist to overtly utilize the Auto-Tune effect within pop music, Dirty South hip hop artist TPain is credited with being the first artist to do the same within hip hop. T-Pain's 2006 debut album Rappa Ternt Sanga ${ }^{57}$ is the first instance where a hip hop artist applied the Auto-Tune Cher-effect to his music. In songs such as "I'm Sprung," "I'm N Luv (Wit a Stripper) (feat. Mike Jones)," and "You Got Me (feat. Akon)," T-Pain applies the Cher-effect as a vocal decoration to both his singing in the chorus and his rapping in the verses. But whereas Cher and her producers used Auto-Tune sparingly and in moderation, T-Pain instead applies Auto-Tune to his entire vocal performance (a la Eiffel 65 and Daft Punk), and places the AutoTune settings at the program's extreme, producing a constant, exaggerated vocal flutter. This unique application of Auto-Tune within T-Pain's hip hop idiom proved commercially successful: the single for "I'm Sprung" reached number eight on the Billboard Hot 100 Radio chart, number thirteen on the R\&B/Hip Hop songs chart,

57 T-Pain, Rappa Ternt Sanga, Sony Music Canada B000BFoDJC, compact disc, 2006. 
while reaching number thirty-three on the Billboard Pop songs chart.58 His 2007 sophomore album Epiphany, ${ }^{59}$ which featured the single "Buy U a Drank (Shawty Snappin') (feat. Yung Joc)" and followed the same heavily Auto-Tuned formula, was even more successful, reaching the number one spot on both the R\&B/Hip Hop songs chart and the Billboard Hot 100 radio chart.60 In light of his commercial and artistic breakthrough, T-Pain is widely recognized as the first artist to explore the application of Auto-Tune within the genre of hip hop. These innovations -- artistic, cultural and technological -- helped to both raise T-Pain's profile as a musician and extend the Auto-Tune Cher-effect further into the zeitgeist.

T-Pain's use of Auto-Tune tends towards a vocal, singing delivery, as opposed to the more conventional rapped delivery of hip hop. The Auto-Tune Cher-effect enables a hip hop artist such as T-Pain to vocally emulate the delivery and ability of a pop music vocalist. By stylistically drawing upon the vocal forms of pop music and incorporating them into a hip hop musical arrangement, T-Pain has effectively created a musical and vocal hybrid. This hyrbid musical form, which combines southern hip hop arrangements with the emulation of pop music-influenced vocality, can be considered generically transgressive: not only does it fuse hip hop music with pop music vocality, it also transgresses the genre

\footnotetext{
58 "T-Pain Album \& Song Chart History," Billboard.com, accessed March 28, 2012, http:// www.billboard.com/artist/t-pain/690156\#/artist/t-pain/chart-history/690156.

59 T-Pain, Epiphany, Sony Music Canada B000P0JQ9Q, compact disc, 2007.

80 "T-Pain Album \& Song Chart History," Billboard.com, accessed March 28, 2012, http:/l ww.billboard.com/artist/t-pain/690156\#/artist/t-pain/chart-history/690156.
} 
values and conventions of modern R\&B music. Musically, hip hop and modern R\&B are relatively similar, in that they both utilize samples, keyboards/ synthesizers, and strong rhythmic foundations in their musical arrangements. The primary and fundamental distinction between these two genres is in their respective vocal deliveries: $R \& B$ music is primarily sung by a skilled vocalist in a soulful, virtuosic, gospel-influenced manner, whereas hip hop is primarily delivered in a rapped, highly rhythmic, spoken word delivery. T-Pain's AutoTuned-MC vocal style can be considered transgressive in that it enables the vocalist to simultaneously delivery lyrics in a rapped and sung manner. However, while T-Pain's unique vocal delivery transgresses the genre boundaries and musical conventions of hip hop and modern $R \& B$, as well as drawing upon pop music vocality, the core of his music fundamentally remains hip hop, given the primacy of the delivery of the lyrics. T-Pain uses Auto-Tune in order to flirt with pop music and R\&B vocality, to stylistically emulate these forms, rather than to recast himself fully as a pop or R\&B musician.

This unique musical fusion sparked a fascination with Auto-Tune amongst the broader hip hop community, inspiring many more hip hop, rap, and even R\&B musicians to experiment with the technology. For example, artists such as Lil Wayne have used Auto-Tune along similar sonic and stylistic lines as T-Pain. In his 2008 hit single "Lollipop," ${ }^{\prime 61}$ Lil Wayne applies the Cher-effect to his unique blend of half-rapped, half-sung lyrics. Canadian hip hop artist Drake, a protégé

\footnotetext{
61 Lil Wayne, "Lollipop," Tha Carter III, Young Money Records Inc./Cash Money Records Inc. B001E4IY3Q, compact disc, 2008.
} 
and member of Lil Wayne's Young Money Entertainment record label, has also used Auto-Tune to blend hip hop and R\&B vocal styles. In songs like "Best I Ever Had, ${ }^{\prime 62}$ Drake applies the Auto-Tune effect within his unique blend of rap, hip hop and R\&B vocal styles, seamlessly transitioning between singing choruses to halfrapped, half-sung verses. What distinguishes Drake from fellow artists Lil Wayne and T-Pain is the degree to which Drake applies the Auto-Tune effect: Drake's use is more restrained than Lil Wayne and T-Pain, who use the Auto-Tune software's pitch correction speed at its fastest settings. In Drake's work, the application of Auto-Tune is less overt, being limited mostly to pitch correcting his vocal tuning in the chorus (which with careful listening reveals traces of rapid pitch correction in his descending vocal lines). Drake (and his producers) set Auto-Tune's pitch correction settings fast enough so that the vocal effect is audible, but not so fast that it produces the erratic, exaggerated robotic effect used by T-Pain and Lil Wayne. Finally, from the realm of R\&B, singer Mary J. Blige's 2009 single "The One"63 featured the singer (and guest Drake) with the Auto-Tune effect applied to her voice. For a singer of Mary J. Blige's caliber, it is clear that the usage of Auto-Tune is fundamentally creative rather than corrective. As Blige herself explains,

'The One' introduces the world to 'Crazy Mary.' 'It was for effect,' [Blige] said of using Auto-Tune. 'I wanted 'Crazy Mary' to get her shine on. She couldn't get her shine with my regular

\footnotetext{
62 Drake, "Best I Ever Had," So Far Gone, Young Money Records Inc./Cash Money Records Inc. B002LVAZO2, compact disc, 2009.

63 Mary J. Blige, "The One (feat. Drake)," Stronger With Each Tear, Geffen Records B002UZ5G7U, compact disc, 2009.
} 
voice. I used the effect. I used it as a telephone effect or reverb. ${ }^{m 4}$

As mentioned earlier (in regards to Eiffel 65 and Daft Punk), the Auto-Tune effect creates a unique vocal character and/or identity which a singer can assume, which is precisely Blige's intention in "The One." Using the Auto-Tune effect allows Blige to create, assume, and experiment with her Auto-Tuned alter-ego "Crazy Mary." Also, as with Lady Gaga in "Monster," Blige's use of Auto-Tune is experimental, a way of playing with a new vocal effect within the context of her own musical idiom.

But when it comes to the application of Auto-Tune in hip hop, one artist stands out as equally distinctive and disruptive: Kanye West. By 2008, Kanye West was one of the most widely recognized, well known, and well regarded hip hop artists and cultural icons thanks to the massive commercial success of his previous albums The College Dropout, Late Registration, and Graduation. His unique $M C$ rap-vocal style, combined with his (in)famously self-aggrandizing ego and personality, have established him as one of the biggest musical and pop culture icons of the last decade. West's music also represents a microcosm for not only the methods and techniques in which Auto-Tune is applied to contemporary hip hop music, but also the critical reactions, opinions, and discourses its use generates (from both music critics and listeners within the hip hop community). Therefore, I shall spend the next few pages detailing West's

64 Shaheem Reid, "Mary J. Blige Defends Auto-Tune: 'To Each His Own', MTV.com, June 11, 2009, accessed February 16, 2012, http:/hww.mtv.com/news/articles/1613803/mary-j-bligedefends-autotune.jhtml. 
most recent work, and explore the impact his use of Auto-Tune has had upon the hip hop genre at large.

On his 2008 concept album 808 s and Heartbreak, ${ }^{65}$ West produced the entire album using Auto-Tune in order to enable himself to sing, akin to a pop music singer. In songs like "Love Lockdown" and "Heartless," West relies upon Auto-Tune to both Cher-effect his voice, and to pitch-correct his substandard vocal ability. While lyrically West is exploring heartbreak and upset, sonically Auto-Tune becomes the muse of the album, simultaneously drawing attention to, and providing him with, a surrogate singing voice, thereby allowing him to overcome his vocal deficiencies. Kanye West, in response to his extensive use of Auto-Tune on the album, replied that "What [Auto-Tune] does for me, if I sing offkey, it really points that out. It points out the bad notes. So what I have to do is sing more perfect." ${ }^{66}$ Unlike T-Pain, who also used Auto-Tune throughout his albums, Kanye West took the integration of Auto-Tune in hip hop to a higher degree, making it the musical centrepiece of 808 s and Heartbreak. Auto-Tune, therefore, serves a dual purpose on $808 \mathrm{~s}$ and Heartbreak. it bolsters West's vocal delivery, while also becoming the conceptual theme of the album.

While artists like T-Pain and Lil Wayne have used Auto-Tune to modify their lyrical delivery, creating a hybrid hip hop MC and pop music vocal form,

\footnotetext{
65 Kanye West, 808s and Heartbreak, Roc-A-Fella Records LLC B001FBIPFA, compact disc, 2008.

66 Ben Rogerson, "Kanye West says Auto-Tune makes him a better singer," MusicRadar.com, December 2, 2008, accessed February 16, 2012, http://www.musicradar.com/news/tech/kanyewest-says-auto-tune-makes-him-a-better-singer-185278.
} 
Kanye West instead utilized Auto-Tune in order to recast himself as an R\&B singer proper. For this, West received both praise and criticism, acceptance and backlash, within the hip hop genre and community as well as the larger pop culture: NPR labelled Kanye West an "Auto-Tune crooner," ${ }^{167}$ Rolling Stone, in its review of the album, cleverly opined that "this noble failure of an album might easily have been a noble success if he had tweaked the Fun-o-Meter just a bit. A slight pitch correction could have done the trick," 68 while Spin Magazine observed that "while his cadences and flows as an MC have improved measurably, his singing calls to mind a rudimentary, indie-rock naif following the melody around blindly. ${ }^{\text {"69 }}$ There even exist online petitions imploring West to abandon the use of Auto-Tune in his music, $^{70}$ (although with a total of 110 signatures, the petition will most likely not have enough clout to influence West's creative endeavours). However, West's conceptual use of Auto-Tune was praised by some reviewers, with the Washington Post recognizing that "it's all done with an artfulness that makes West sound more like a brokenhearted android than some anonymous

\footnotetext{
${ }^{67}$ Patrick Jarenwattananon, "Kanye West, Auto-Tune Crooner," NPR, November 30, 2008, accessed February 16, 2012, http://www.nproro/blogs/sundaysoapbox/2008/11/ kanve west autotune crooner $1 . \mathrm{html}$.

68 Jody Rosen, "Kanye West: 808s and Heartbreak Review," Rolling Stone, December 11, 2008, accessed February 16, 2012, http://www.rollingstone.com/music/albumreviews/808sheartbreak-20081211.

69 Charles Aaron, "Album Review: Kanye West's '808s and Heartbreak," Spin Magazine, November 25, 2008, accessed February 16, 2012, http://uwwwspin.com/blog/album-review-kanyewests-808s-and-heartbreak.

70 Bradley Petit-Frere, "Against Kanye West's Use of the Auto Tune," PetitionOnline, accessed February 16, 2012, http:/luww.petitiononline.com/mrwest/petition.html.
} 
pop-throb struggling to stay on pitch, ${ }^{71}$ while one blogger at $X X L$ Magazine, a preeminent hip hop publication, not only praised the album's artistic vision, but defended it against its many critics:

For the most part, traditional rap fans panned the AutoTunefilled disc because there was more singing than rapping, but, personally, I loved it. In fact, I even professed my love for the disc before the album even dropped.

Now, I know I'm in the minority but 808s \& Heartbreak might just be my favorite Kanye album. Yeah, it was emo-heavy and the man can't really sing, but the music was insane and what he was saying was some of the realest stuff to me. It's kind of like what Murs said in his [blog] a few weeks ago, it's like American society refuses to accept a Black man expressing anything other than anger.

Well, I do and I embrace different. ${ }^{72}$

There are a number of reasons why public and critical reactions to $808 \mathrm{~s}$ and Heartbreak were so pointed, ranging from personal distaste for the Auto-Tune effect, West's attempt to portray himself as a singer, to the huge musical and creative shift it represented to both Kanye West's music, and the hip hop genre itself. West, who received his first big musical break as a producer providing beats for Jay-Z's now classic hip hop album The Blueprint, ${ }^{73}$ quickly signed to Jay-Z's Roc-A-Fella record label, becoming a protégé of Jay-Z's. This close affiliation with Jay-Z, as well as collaborations with artists Common, Talib Kweli, Mos Def, and Dilated Peoples, conferred an old-school, "conscious" hip hop

\footnotetext{
71 Chris Richards, "808s and Heartbreak by Kanye West," The Washington Post, November 24, 2008, accessed February 16, 2012, http://www.washingtonpost.com/wp-dyn/content/article/ 2008/11/23/AR2008112302506.html.

72 NWSO, "What's Kanye West's Third Best Album? - "808s" Don't Count," XXL Magazine, July 29, 2010, accessed February 16, 2012, htto:/www xx/mag,com/bloggers/2010/07/what's-kanyewest's-third-best-album-" $808 s^{\prime}$-don't-count/comment-page-4/.

73 Jay-Z, The Blueprint, Universal Music Group B00005054T, compact disc, 2001.
} 
reputation upon West, one that espouses an aesthetic of jazz-sampled beats, socially and/or politically pertinent lyrics, and an aversion to the dominant trends of mainstream hip hop. What 808 s and Heartbreak represented to some in the hip hop community was one of its most successful and talented stars changing his affiliation, converting from the conscious hip hop aesthetic and adopting this new vocal effect, itself the increasingly dominant trend in mainstream hip hop. It forced the genre to address and discuss precisely what role and place Auto-Tune has within the genre, as well as the effect that effect has upon their music.

While West's use of Auto-Tune on the entirety of $808 \mathrm{~s}$ and Heartbreak was controversial, his follow-up 2010 album My Beautiful Dark Twisted Fantasy ${ }^{74}$ featured a more moderate use of Auto-Tune. On songs like "Hell of a Life," he uses the effect more sparingly, containing its use primarily to his singing lines in the chorus, whereas in "Blame Game (feat. Jon Legend)," West begins to explore and experiment with vocal DSP effects other than Auto-Tune (particularly in the second verse, 2:30-3:27). In its review of the album, Pitchfork Media addressed the emotive effectiveness of West's use of DSP vocal effects within the song: "Kanye's voice is sped up, slowed down and stretched out. The effect is almost psychotic, suggesting three or four inner monologues fighting over smashed emotions. ${ }^{75}$ On "Lost in the World (feat. Bon Iver)," the use of Auto-Tune is

\footnotetext{
74 Kanye West, My Beautiful Dark Twisted Fantasy, Roc-A-Fella Records LLC/Def Jam B003X2O6KW, compact disc, 2010.

75 Ryan Dombal, "Kanye West: My Beautiful Dark Twisted Fantasy Album Review," Pitchfork Media, November 22, 2010, accessed February 16, 2012, http://pitchfork.com/reviews/albums/ 14880-my-beautiful-dark-twisted-fantasyl.
} 
instead derived from a sample of "Woods, ${ }^{76}$ a song which itself is an experimental Auto-Tuned track by alternative, indie-folk artist Bon Iver. "Woods," which starts with a single, Auto-Tuned vocal, progresses by adding layer upon layer of further Auto-Tuned vocal harmonies. West samples these Auto-Tuned vocal harmonies, making it the vocal and melodic foundation for the track, to which West further applies his own vocal harmonies, melodies, and rhythms atop the mix. On "Runaway," the album's 9-minute extravaganza, West applies AutoTune and a fuzz-box effect pedal to his voice during the song's 3-minute outro. Accompanied by a vamping string quartet and piano, West generates a truly unique sound effect on his vocals, one which enables him to instrumentalize ${ }^{77}$ his voice, "making himself wail like a dying cyborg."78 The result is a truly astonishing, convention breaking recording: instead of Auto-Tune being utilized to correct and/or add effect to a recorded vocal, West's use of Auto-Tune in "Runaway" recontextualizes his voice into a soloing instrument, which he uses to musically express himself. As well, the absence of Auto-Tune is noticeable in parts of the album, particularly on "Dark Fantasy," where Kanye's off-key, out-oftune delivery (at 2:38) stands out as intentionally and deliberately free of AutoTune.

\footnotetext{
78 Bon Iver, "Woods," Blood Bank (EP), jagjagwar B001MJ3MQW, compact disc, 2009.

77 "instrumentalize," Merriam-Webster Dictionary, accessed March 27, 2012, http://www.merriamwebster.com/dictionary/instrumentalize.

78 Ryan Dombal, "Kanye West: My Beautiful Dark Twisted Fantasy Album Review," Pitchfork Media, November 22, 2010, accessed February 16, 2012, http://pitchfork.com/reviews/albums/ 14880-my-beautiful-dark-twisted-fantasyl.
} 
While My Beautiful Dark Twisted Fantasy received widespread praise and acclaim from publications as diverse as $H i p H o p D X,{ }^{79} X X{ }^{2}$ Magazine, ${ }^{80}$ and even Rolling Stone, ${ }^{81}$ many within the hip hop community nevertheless continued to disapprove of the use of Auto-Tune within the genre. One of the most vocal opponents to hip hop's use of Auto-Tune turned out to be none other than Kanye West's mentor, Jay-Z, whose 2009 single "D.O.A. (Death of Auto-Tune), represented a defiant and dissenting voice within hip hop towards the use of Auto-Tune. As Jay-Z's lead single from his 2009 album The Blueprint III, “D.O.A. (Death of Auto-Tune)" condemned the excessive use of Auto-Tune within hip hop, and called upon the genre to abandon the technology in favour of returning to what in his opinion it does best, rapping. As one of hip hop's most respected artists and MCs, Jay-Z raps in the third verse that "Y'all niggas singing too much / Get back to rap / you T-Pain'in too much," a criticism regarding contemporary hip hop's misguided fascination with Auto-Tune. Also, to match the song's theme of opposition to the increasing use of Auto-Tune within hip hop, Jay-Z, during the song's chorus, sings the popular sports arena anthem "Na Na Hey Hey Kiss Him

\footnotetext{
79 Jake Paine, "Kanye West's My Beautiful Dark Twisted Fantasy," HipHopDX, November 15, 2010, accessed February 16, 2012, http://www.hiphopdx.com/feature/30in30/page8.php.

80 Jesse Gissen, "Recovery or My Beautiful Dark Twisted Fantasy?: Which Is the Best Album of 2010?," XXLMagazine, December 7, 2010, accessed February 16, 2012, http:/www.xxlmag.com/ bloggers/2010/12/recovery-or-my-beautiful-dark-twisted-fantasy/.

B1 "30 Best Albums of 2010," Rolling Stone, accessed February 16, 2012, http:ll uww.rollingstone.com/music/lists/30-best-albums-of-2010-20101213/kanye-west-my-beautifuldark-twisted-fantasy-19691231.

82 Jay-Z, "D.O.A. (Death of Auto-Tune)," The Blueprint III, Roc-a-Fella Records LLC/Warner Music Group B002DMJM66, compact disc, 2009.
} 
Goodbye" by Steam ${ }^{83}$ completely off-key. In this instance, the absence of AutoTune is precisely Jay-Z's point: not only does the chorus of Steam's song lyrically play into Jay-Z's disapproval of Auto-Tune, but the fact that he then sings the passage off-key is intentionally antagonistic. In Jay-Z's hip hop aesthetic, rappers and MCs are not meant to be gifted singers, they are meant to do what they do best: rap. Musically, Jay-Z's off-pitch vocal delivery is jarring to listen to; symbolically, the presence of intentionally off-pitch vocals is Jay-Z's political statement to the hip hop community that both pitch correction and vocal effects have no place within hip hop aesthetics. While Jay-Z's sentiments resonated amongst many within the hip hop community, the use of Auto-Tune has continued to progress, and shows no sign of ceasing in the near future.

Other artists have also released music that has echoed this defiant sentiment: KRS-ONE's "Robot (feat. Buckshot)" is an anti-Auto-Tune (and DSP) song in the same vein as Jay-Z's "D.O.A (Death of Auto-Tune)." Legendary rapper KRS-ONE and his young protégé Buckshot direct their criticism towards the misplaced artistic and musical values amongst the pro-Auto-Tune sect of hip hop: "you cats got it backwards / you say real is whack / and the fake shit, that's good! / well, keep on fakin' / we'll just keep on makin." 84 In this final verse, we see the aesthetic values of the two camps delineated: according to KRS-ONE and Buckshot, the pro-Auto-Tune artists' sense of musical taste is inverted, that

\footnotetext{
83 Steam, "Na Na Hey Hey Kiss Him Goodbye," Steam, Fab Distribution B0000DIJLW, 2003, compact disc, originally released in 1969.

84 "KRS-ONE \& BUCKSHOT "ROBOT" MUSIC VIDEO," YouTube video, 4:00, uploaded by duckdown on July 8, 2009, accessed February 16, 2012, http://uww.youtube.com/watch? $v=$ i8RagDs03c4\&feature $=$ player embedded.
} 
they value "fake" music over "real" music, whereas KRS-ONE and Buckshot declare that they will keep on "makin" (i.e. real music). Of course, what makes this discourse over "fake" and "real" hip hop music so contentious is that both sides believe that they are making "real" hip hop, and that their critics are "fake." What specifically informs KRS-ONE's notions and perspectives on what makes hip hop music "real" rather than "fake" is his appeal to a particular old-school hip hop vocal aesthetic, also espoused by Jay- $Z$, in which the MC delivers his lyrics in a rap vocal form. Sugarhill Gang's "Rapper's Delight," ${ }^{85}$ Grandmaster Flash and the Furious Five's "The Message,"86 and Public Enemy's "Fight the Power,"87 are all prime examples of the old-school, MC rap vocal aesthetic to which KRSONE and Jay-Z are appealing in their dismissal of the new and emerging vocal style and aesthetic that Auto-Tune presents in modern hip hop.

However, signs of reconciliation and integration are beginning to emerge, as evidenced by the 2011 album Watch the Throne,88 a joint collaboration between Jay- $Z$ and Kanye West. The album attempts to reconcile the stylistic incongruities between the new-school pop vocal use of Auto-Tune and the oldschool adherence to conventional rapped vocal delivery, looped musical instrumentation, and structure. For instance, in songs like album opener "No

\footnotetext{
${ }^{85}$ Sugarhill Gang, "Rapper's Delight," Rapper's Delight: The Best of the Sugarhill Gang, Warner Music Group B0000033K4, compact disc, 1996, originally released in 1980.

86 Grandmaster Flash and the Furious Five, "The Message," The Message, Sugar Hill Records B00006F2XV, compact disc, 2002, originally released in 1982.

87 Public Enemy, "Fight The Power," Fear of a Black Planet, Def Jam Recordings B0000024IE, compact disc, 1990.

86 Jay-Z and Kanye West, Watch the Throne, Roc-A-Fella Records LLC/Universal Music B005BQLCBO, compact disc, 2011.
} 
Church in the Wild (feat. Frank Ocean)" the instrumental background track features a more conventional structure, a motif that is repeated throughout the verses, broken up by a synthesizer led turnaround, which provides a stable, repeating foundation for both Jay- $Z$ and Kanye West to rap overtop of. What makes "No Church in the Wild" unique is its second verse, where guest vocalist Frank Ocean sings in the heavily Auto-Tuned, new-school style of T-Pain and Kanye West; within the same song, elements of both old-school rap and newschool hip-pop are fused together. This theme is repeated throughout the album on songs like "New Day," where Nina Simone's voice is sampled and Auto-Tuned to form the melody used for the song's chorus, while Jay- $Z$ and Kanye West follow the old-school tradition of rapping over the verses. In "Welcome to the Jungle," Kanye West can be heard adding Auto-Tuned vocal flourishes to the turnaround in the verse (at $1: 25$ ), although his vocals are buried in the mix, with Jay-Z's rap given primary focus.

To Kanye West and Jay-Z's credit, the album manages to find a neutral yet common ground between the two aesthetics: it manages to incorporate the AutoTune pop vocal aesthetic with the old-school MC tradition in an attempt to reconnect with the foundation of hip hop. While it attempts to resolve the conflict between the two recently divergent schools of hip hop, one particularly interesting and noticeable aspect about this album is that at no point is Jay-Z Auto-Tuned. This aspect shows that, while Jay-Z may not as ardently disapprove of Auto-Tune in hip hop as he previously did, he continues to tacitly affirm his affiliation with the old-school hip hop MC aesthetic. While Watch the Throne may 
not signify hip hop's widespread acceptance of Auto-Tune, it does demonstrate a growing tolerance for -- and integration with -- the software and effect within the genre.

\section{Hip-Pop}

As mentioned previously, the import of Auto-Tune into hip hop has held a number of stylistic and aesthetic ramifications for the genre: it has challenged the dominance of the old-school rapped vocal aesthetic, positing a new form of hip hop vocals. In this new model, the MC is transformed from a spoken word rap lyricist into a more pop vocal influenced singer. The use of Auto-Tune in this new model is crucial: not only does it enable the artist to sing, but it affects the artist's voice in such a way that it in essence provides him with a new singing voice. Auto-Tune not only challenges the vocal form of hip hop, it also affects the musical form of hip hop as well.

It should be noted that the blending of hip hop, R\&B and pop music forms and conventions has been occurring independently of Auto-Tune for a number of years. Since the 1990s, a number of artists have been experimenting with and emulating a variety of musical conventions, taken from pop music, R\&B, hip hop, and establishing a hip hop/R\&B hybridization. For example, Janet Jackson's "Got 'Til It's Gone" 89 blends a hip hop remix of Joni Mitchell's "Big Yellow Taxi"90 with

\footnotetext{
89 Janet Jackson, "Got 'Til It's Gone," The Velvet Rope, EMI Music Canada B000000WEX, compact disc, 1997.

${ }^{00}$ Joni Mitchell, "Big Yellow Taxi," Ladies of the Canyon, Warner Brothers Music/Reprise B000002KOQ, compact disc, 1990, originally released in 1970.
} 
Jackson's R\&B vocality (and vocal overdubs from $A$ Tribe Called Quest MC QTip), Lauryn Hill's "Doo Wop (That Thing) ${ }^{\text {m1 }}$ blends R\&B instrumentation with hip hop MCing, while Gnarls Barkley's "Crazy, ${ }^{\text {"92 }}$ a collaboration between R\&B vocalist Cee Lo Green and hip hop producer Danger Mouse, fuses Green's virtuosic and soulful voice with Danger Mouse's hip hop production. While the blending of pop music, R\&B, and hip hop musical forms have been occurring for a number of years, the recent introduction of Auto-Tune into hip hop has led to the expansion of the hip hop MC vocal style through the stylistic emulation of pop music vocality. This vocal metamorphosis and expansion is reflected in T-Pain's music as well: his music highlights an increasing intersection between hip hop and pop music by emulating a number of pop music characteristics, such as melody and harmony, the extensive use of hooks, the use of more conventional verse-chorus structures (a la pop music), and the recontextualizing of the $M C$ as a singer.

This increased intersection between pop music and hip hop has been colloquially referred to as hip-pop: a hybrid musical form of modern, mainstream hip hop that draws upon a number of musical and stylistic influences from pop music. While Auto-Tune has assisted in the modern formation and iteration of this hybrid subgenre, the development of this hip hop and pop music fusion has been in motion for a number of years (as the above examples illustrate). It should also

\footnotetext{
91 Lauryn Hill, "Doo Wop (That Thing)," The Miseducation of Lauryn Hill, Sony Music Canada B00000ADG2, compact disc, 1998.

92 Gnarls Barkley, “Crazy,” St. Elsewhere, Atlantic Records B000F3AAUW, compact disc, 2006.
} 
be noted that while the influence between hip hop music and pop music is reciprocal, it is not entirely equal: while hip hop music has recently drawn a great deal of musical and stylistic influence from pop music, pop music has also been influenced by hip hop, but most commonly in the form of guest-appearance collaborations. But before that aspect can be explored, it is necessary to first discuss the ways in which hip hop is stylistically emulating pop music.

As mentioned previously, artists like T-Pain, Lil Wayne and Drake, are not only incorporating Auto-Tune into their music, but recasting themselves as hip hop singers, as well as MCs. These artists are also colleagues, fellow labelmates on Lil Wayne's Cash Money record label (a subsidiary of Lil Wayne's Young Money Entertainment production company), who have collectively garnered a reputation over the last several years as innovators of hip hop's newest sounds and trends. As one of Young Money Entertainment's most promising -- and heavily promoted ${ }^{93}$.- artists, Drake in particular finds himself stylistically situated within the burgeoning crossover between pop and hip hop. With very talented MC skills and clever lyrics, Drake's music can be considered hip hop with a pop music appeal. His tendency to croon and explore themes of love and difficult romantic relationship dynamics exposes a more gentle quality to his character, one at odds with the hard, aggressive old-school MC character. Drake's sensitivity, in both his lyrics and singing style, are indicative of a pop music sentimentality, one that rarely finds articulation within old-school hip hop.

\footnotetext{
93 Edwyn Huang, "Hate It Or Love It: A Hip Hop Analysis Of Drake," HipHopDX, August 29, 2009, accessed February 16, 2012, http://www.hiphopdx.com/index/editorials/id.1398/title.hate-it-orlove-it-a-hip hop-analysis-of-drake.
} 
When it comes to the increasing hybridization of hip hop and pop music into a new subgenre of popular music, fellow Young Money Entertainment labelmate Nicki Minaj stands out. Minaj's debut album Pink Friday ${ }^{94}$ is a diverse collection of hip-pop music, with songs such as "Roman's Revenge (feat. Eminem)" and "Blazin' (feat. Kanye West)" presenting a harder, hip hop edge with explicit lyrics and themes, while songs like "Moment 4 Life (feat. Drake)," "Fly (feat. Rihanna)" and "Super Bass" feature hook-heavy pop music melodies and structures. While Minaj's manic, aggressive MC rap style anchors her work in the realm of hip hop, her music's tendency towards conventional verse-chorus structures, guest vocalists, and catchy, hook-heavy choruses is more reminiscent of pop music. This alchemy is particularly prevalent on her collaboration with Black Eyed Peas frontman will.i.am on "Check It Out," a song which heavily samples The Buggle's "Video Killed the Radio Star." ${ }^{\text {95 }}$ Musically, the song is an ideal example of hip-pop: it incorporates a heavy use of synthesizers, samples, Auto-Tune (nearly every bar of will.i.am's vocals throughout the song are applied with the Cher-effect, whereas Nicki Minaj is only occasionally Auto-Tuned, presumably for lyrical emphasis), a traditional pop music verse-chorus structure, and the combination of both new-school singing and old-school MCing (Minaj's primary role within the song). As this song demonstrates, the Auto-Tune effect is a key component in the sound of this burgeoning hip-pop idiom: it distinguishes it

\footnotetext{
94 Nicki Minaj, Pink Friday, Young Money Entertainment/Cash Money Records Inc. B0049D1TCM, compact disc, 2010.

95 The Buggles, "Video Killed the Radio Star," The Age of Plastic, Island/Universal B000025709, compact disc, 2008, originally released in 1979.
} 
from the conventional musical instrumentation of old-school hip hop, placing it into a new, unique musical context, to which artists such as will.i.am and Nicki Minaj are eager to explore and expand.

Commercially speaking, the musical group that has emerged as the most successful and recognized purveyors of hip-pop is the Black Eyed Peas. Songs such as "Boom Boom Pow," "The Time (Dirty Bit), ${ }^{n 97}$ and "I Gotta Feeling," all examples of the increasing hybridization of hip hop with pop music. While the Black Eyed Peas incorporate the Auto-Tune Cher-effect in a manner similar to TPain, their music stylistically draws upon -- and is more evidently influenced by -the musical and genre conventions of both pop music and electronic music. "Boom Boom Pow," in particular, combines the idiosyncratic Auto-Tuned MC vocal delivery of new-school hip hop with the instrumentation and vocal template established by electronic music groups like Eiffel 65 and Daft Punk. The Black Eyed Peas' music represents an increasing cross-pollination between pop music, hip hop, and electronic music with considerable success: not only have their albums sold millions of copies, but a number of their singles hold the all-time, most purchased singles spots in iTunes' 10 ten list. ${ }^{99}$ As the Black Eyed Peas' music demonstrates, the utilization of Auto-Tune within a highly pop and dance

\footnotetext{
96 Black Eyed Peas, "Boom Boom Pow," The E.N.D., Interscope B00192IVOO, compact disc, 2009.

${ }^{97}$ Black Eyed Peas, "The Time (Dirty Bit)," The Beginning, Interscope B00475AQIU, compact disc, 2010.

98 Black Eyed Peas, "I Gotta Feeling," The E.N.D., Interscope B00192IVOO, compact disc, 2009.

99 MTV News Staff, "Black Eyed Peas, Lady Gaga Top iTunes All-Time Most-Downloaded Songs," MTV.com, February 11, 2010, accessed February 16, 2012, http://www.mtv.com/news/articles/ 1631774/bep-aaga-top-itunes-alltime-mostdownloaded-songs.jhtml.
} 
music influenced idiom of hip hop can be hugely successful, both commercially and culturally.

Pop music's stylistic emulation of hip hop has been more measured, however: the general tendency within pop music is to dedicate either a verse or bridge to the guest $\mathrm{MC}$, essentially isolating them from the rest of the song, but nonetheless lending the song a degree of hip hop credibility. Examples abound of this type of hip-pop collaboration: Katy Perry does this twice, first with Snoop Dogg in the final verse of "California Gurls (feat. Snoop Dogg)," and again in her remix collaboration with Kanye West on "E.T."100 (although Kanye West is generously given two separate verses in the song). Jay-Z provides a verse in Beyoncé's "Crazy in Love,"101 as well as in the opening verse on Rihanna's "Umbrella." J02 Justin Bieber's "Baby"103 features guest MC Ludacris, Alyssa Reid's "Alone Again"104 features a brief verse from rapper P. Reign, and Rebecca Black's "Friday" features Patrice Wilson, the songs' producer and Ark Music Factory executive. Although the frequency at which pop musicians are collaborating with hip hop artists may be increasing, hip hop's musical forms

\footnotetext{
100 Katy Perry, "E.T. (feat. Kanye West)," Teenage Dream: The Complete Collection, EMI Music Canada B006XBSAP2, compact disc, 2012, originally released in 2011.

101 Beyoncé, "Crazy in Love (feat. Jay-Z)," Dangerously in Love, Sony Music Canada B000099T2L, compact disc, 2003.

102 Rihanna, "Umbrella (feat. Jay-Z)," Good Girl Gone Bad, The Island/Def Jam Music Group B0018S6YO4, compact disc, 2008.

100 Justin Bieber, "Baby (feat. Ludacris)," My World 2.0, The Island, Def Jam Music Group B003AIBGLG, compact disc, 2010.

104 Alyssa Reid, "Alone Again (feat. P. Reign)," The Game, Warner Music Group B004ZKCGNA, compact disc, 2011.
} 
have arguably had less of an impact upon pop music than pop music has had on hip hop. The general tendency amongst many pop musicians is to collaborate with hip hop artists within the context of their own pop music idiom, rather than emulate or incorporate the hip hop musical forms explored by these artists in their own respective work.

However, one artist in particular demonstrates how successfully, both artistically and commercially, pop music and hip hop music forms can be fused together: Justin Timberlake. Songs like "SexyBack,"105 "What Goes Around (Comes Around)"106 and "Chop Me Up"107 (which features hip hop group Three 6 Mafia), are prime examples of how pop music and hip hop can be combined to form a popular and potent musical blend. Timbaland, Justin Timberlake's producer for FutureSex/LoveSounds, is also a prime example of how pop music and hip hop can be fused together into a hybrid hip-pop form. Timbaland's music embodies the creative crossover between pop music, hip hop and R\&B, infusing pop music soundscapes with hip hop forms and influences. This fusion is emphasized in his various collaborations with top-tier pop musicians, particularly in songs such as "The Way I Are (feat. Keri Hilson),"108 "Give It To Me (feat.

\footnotetext{
105 Justin Timberlake, "SexyBack," Futuresex/Lovesounds, Sony Music Canada B000H305U0, compact disc, 2006.

106 Justin Timberlake, "What Goes Around (Comes Around)," FuturesexLovesounds, Sony Music Canada B000H305UO, compact disc, 2006.

107 Justin Timberlake, "Chop Me Up," Futuresex/Lovesounds, Sony Music Canada B000H305U0, compact disc, 2006.

108 Timbaland, "The Way I Are (feat. Keri Hilson \& D.O.E.)," Shock Value, Universal Music Group B000NA26ZE, compact disc, 2007.
} 
Justin Timberlake and Nelly Furtado)" 109 "If We Ever Meet Again (feat. Katy Perry), ${ }^{110}$ and "Say Something (feat. Drake), ${ }^{m 111}$ all of which feature Timbaland's distinct blend of pop music and hip hop forms, styles, and influences.

\section{Conclusion}

In Chapter II, I explored not only the debut and development of a variety of new analog and digital musical instrument technologies, but also the impact those new technologies had on the field of popular music. In this chapter, I have demonstrated the ways in which Auto-Tune, one of the more recent music technology innovations, has similarly altered the musical landscape into which it has been introduced, and how it -- like many other music technologies before it -led to the formation of new musical practices and subgenres. In this case, AutoTune has contributed to the increasing cross-pollination between pop music and hip hop.

The emergence of this subgenre, however, has not been without conflict and resistance: Auto-Tune has prompted a number of contentious issues and debates within the music industry, particularly amongst artists, producers, and engineers who continually debate over the application of Auto-Tune, since its usage affects the context of the music to which it is applied. Hip Hop has demonstrated a particularly vocal resistance, and a great deal of internal strife,

\footnotetext{
109 Timbaland, "Give It To Me (feat. Nelly Furtado \& Justin Timberlake)," Shock Value, Universal Music Group B000NA26ZE, compact disc, 2007.

110 Timbaland, "If We Ever Meet Again (feat. Katy Perry)," Shock Value II, Blackground Records/ Interscope B002SGQGYI, compact disc, 2009.

111 Timbaland, "Say Something (feat. Drake)," Shock Value II, Blackground Records/Interscope B002SGQGYI, compact disc, 2009.
} 
regarding the import of Auto-Tune. The occasionally heated discourse generated by the use of Auto-Tune has effectively created a schism within the hip hop community. There is, however, a certain irony to the old-school sect's criticism of Auto-Tune: this same group of hip hop artists who, in the earlier part of their career, experimented with and enthusiastically adopted new digital music technologies such as sampling now find themselves opposed to younger hip hop artists conducting themselves in a very similar manner, only in this instance with Auto-Tune.

Pop music, however, has proven considerably more open to, and accepting of, Auto-Tune. Given pop music's historical tendency towards becoming an early adopter of new music and studio technologies, it is quite understandable that pop music would not only quickly, but enthusiastically adopt new vocal technologies. Also, Auto-Tune does not pose the same musical and aesthetic conflicts for pop music as it does for hip hop because the program's ability to produce perfect tuning and pitch greatly appeals to the aesthetic principles and musical values of pop music, which traditionally emphasize vocal precision and high studio production values. A more detailed and nuanced exploration of these values, which underlie the aesthetic principles and priorities of both genres, will be the focus of Chapter IV. 


\section{IV - Popular Music Vocal Aesthetics and Auto- Tune}

In Chapter III, I explored the various methods and techniques through which Auto-Tune has been applied by pop music and hip hop musicians, and the variety of reactions and responses -- both positive and critical -- that the use of Auto-Tune can illicit. So far, much of this thesis has been founded upon the assumption that the utilization of, and reaction to Auto-Tune breaks down not just along genre lines but according to genre specific values. Within pop music, the use of Auto-Tune has, generally speaking, been well suited: the technology provides a means by which producers and engineers can quickly, easily, and non-destructively edit a pop vocalist's performance to produce a pitch-perfect pop music vocal recording. This ability is appealing to many within the pop music genre because the technology complements a number of the genre's core aesthetic values; whereas in hip hop, Auto-Tune conflicts with some of the genre's established, old-school aesthetic ideals. In this chapter, I shall identify and explore in greater detail precisely what aesthetic principles Auto-Tune either complements or conflicts with in both pop music and hip hop, with the intention of determining to what degree genre aesthetics and conventions inform Auto-Tune's reception.

I shall also examine in this chapter pop music's and hip hop's unique relationship to music technology, in order to understand each genre's aptitude for,

and response to, Auto-Tune. However, before I explore the aesthetic values of pop music, I must first make the distinction between pop music and popular 
music more generally, in order to establish pop music as a distinct subgenre with its own musical, aesthetic and stylistic values.

\section{Popular Music vs. Pop Music}

Popular music in the contemporary sense is often said to have emerged in the late $19^{\text {th }}$ to early $20^{\text {th }}$ century, in part as a historical and stylistic distinction from what we now refer to as classical music. In terms of specific musical values and traits that distinguish popular music vocality from classical music vocality, the New Grove Dictionary of Music and Musicians ${ }^{112}$ identifies four key values for a variety of $20^{\text {th }}$ century singing styles, including popular music: first, the experimentation with new sounds in all forms of new music; second, changes in the physiology of singing, particularly "the retreat from the $19^{\text {th }}$-century sensation of pulling the chest voice into the upper register (with a subsequent reduction in vibrato), ${ }^{113}$ which can be found occurring in popular folk singing; third, the preference for high voices, such as the falsettist in popular music; finally, "the dramatic rise in so-called crossover projects [which] has led to various kinds of transference." ${ }^{114}$ These vocal traits, in conjunction with many other musical and socio-economic factors -- such as the rise of commercial sheet music as a major industry -- helped to demarcate the historical and stylistic boundary between earlier musics and what would become considered popular music in the early

\footnotetext{
112 "Singing: $20^{\text {th }}$ Century," in The New Grove Dictionary of Music and Musicians, Second Edition, Volume 23, ed. Stanley Sadie, executive ed. John Tyrrell, (London: Macmillan Publishers Limited, 2001), 433-434.
}

113 "Singing: 20"t Century," The New Grove Dictionary of Music and Musicians, 434.

114 "Singing: 20th Century," The New Grove Dictionary of Music and Musicians, 434. 
part of the $20^{\text {th }}$ century. These popular music tunes experimented with new sounds and melodies (such as the blue note, taken from early ragtime, jazz, and blues music), as well as compositional structures (such as the now universal verse-chorus song structure) which were distinct in comparison to much classical and folk music. As well, early forms of popular music fostered new physiological forms of singing: instead of having to project with great diaphragmatic strength (due to acoustic necessity in large concert halls), singers of popular music were able to explore and experiment with new vocal timbres, ranges, and techniques that classical singers were unable to. Finally, the influence of early ragtime, jazz and blues music upon the composers of early popular music crossed over into their compositions, generating a transference of style the resulted in a unique musical blend, as is heard in the melodies of many Tin Pan Alley compositions.

These attributes have also proven to be remarkably pertinent in the case of modern, $21^{\text {st }}$ century pop music and hip hop: both pop music and hip hop have, in their own right, experimented with new sounds (particularly with the Auto-Tune effect) and have seen changes in the physiological act of singing. Auto-Tune has also partly contributed to an increase in high octave voices in hip hop, most likely primarily because the Auto-Tune effect is much more pronounced and audible when the pitch correction occurs in the upper ranges. Such is the case in Kanye West's "Love Lockdown," where in the opening verse West sings in his lower octave range, which produces only small audible pitch correction effects. However, in the song's chorus, the Auto-Tune effect is more pronounced in West's vocals, his upper vocal range accentuating the program's 
rapid pitch correction settings. Finally, the transference that occurs through crossover projects can be seen in the way hip-pop artists, in their use of AutoTune, not only utilize pop music techniques and methods of applying Auto-Tune to their vocals, but also emulate and borrow various stylistic tendencies from amongst pop music vocalists, such as decorative flourishes.

\section{Pop Music}

With regard to the task of distinguishing the subgenre of pop music relative to popular music more generally, it must be stated that there is no single, universally accepted or widely agreed-upon definition of pop music. Pop music has been recognized through various historical eras and stylistic iterations, which is why a definition and/or examination of pop music will -- inevitably -- require a certain amount of exclusion and/or personal choice. With that in mind, I shall explore a specific vocal form of pop music which began as early as the late 1920 s and rose to prominence during the 1950s and 1960s. This form of pop music explored the relationship between the singer and the microphone, spawning the vocal style/technique referred to as crooning. This vocal technique can be considered the historical and stylistic precursor to the modern form of pop and $\mathrm{R}+\mathrm{B}$ vocal crooning to which Auto-Tuned hip hop aspires. This particular idiom of pop music vocality was also associated with a stream of teen-oriented pop music in the 1950s and 1960s, with musicians such as Bobby Vee, Ricky Nelson, and Paul Anka, in addition to the earlier, more adult-oriented form of vocal pop music which featured a similarly intimate, if more mature, form of vocal crooning (as practiced by musicians such as Frank Sinatra, Bing Crosby, and 
Perry Como). However, with the emergence of Rock and Roll in the early-to-mid 1950s,

pop is seen to have emerged as a somewhat watered-down, blander version of [Rock and Roll], associated with a more rhythmic style and smoother vocal harmony - the period of teen idols in the late 1950s and early 1960s (e.g. Bobby Vee). Subsequently the term was used to characterize chart and teenage audience-oriented music...115

Pop music's general stylistic preferences for smoother vocal harmonies, as well as a less aggressive tendency (musically, lyrically, and visually), represented a stylistic and aesthetic counterpoint to the raucous, subversive musical style of Chuck Berry, Little Richard, Jerry Lee Lewis, and other early Rock and Roll musicians. Whereas Rock and Roll promoted aggressive, passionate, rebellious attitudes and performances, "musically [Pop] is defined by its general accessibility, an emphasis on memorable hooks, and a pre-occupation with romantic love as a theme."116

However, unlike Rock and Roll, pop music vocality has its historical roots in the early part of the $20^{\text {th }}$ century with the invention and adoption of electrified, amplified, and recorded sound. This innovation effectively changed not only the methods and techniques by which singers sang, but also led to changes in musical style which conversely helped form a variety of $20^{\text {th }}$ century notions of popular music and pop music:

\footnotetext{
115 Roy Shuker, "Pop Music," Key Concepts in Popular Music, (London/New York: Routledge, 1998), 226.

116 Shuker, “Pop Music," 226.
} 
Techniques of electronic amplification, including the microphone, have, especially in popular singing, altered vocal production, further dividing the 'classical' and 'popular' singer. Without the need to project the voice naturally over robust (and often amplified) accompaniments in vast halls, popular singers, such as Billie Holiday and Frank Sinatra, did not need to power their voices physically (through diaphragmatic support, use of chest voice and breath control), but could make previously inaudible intimate vocal nuances, such as whispers or murmurs, audible in live performance. ${ }^{117}$

Because electronic amplification enabled singers to sing in a way that did not require the same degree of projection as was demanded from still-unamplified classical singers, a new vocal form emerged that enabled a new generation of singers to stylistically distinguish themselves from classical singers, as well as to explore and experiment with an electronic technology which fostered a new relationship between the singer and the voice. The microphone, because it substituted physiological projection with electronically amplified projection, enabled popular singers to explore and experiment with a whole new range of vocal forms and dynamics. Twentieth-century popular music emerged, and distinguished itself from classical music, through the new singing dynamics that resulted from electronic amplification. The significance of the microphone in pop music was that it "enabled singers to dispense with the mechanisms of projection and retain the nuances of speech, thereby seeming to create a feeling of a oneto-one relationship with their listeners. ${ }^{1118}$ What the pop music vocalists of the 1950s -- both teen and adult oriented -- explored was the ability of the microphone to create a level of intimacy between the singer and the audience; to

\footnotetext{
117 "Singing: $20^{\text {th }}$ Century," The New Grove Dictionary of Music and Musicians, 432-433.

118 "Singing: $20^{\text {th }}$ Century," The New Grove Dictionary of Music and Musicians, 434.
} 
convey not just a lyrical text, but also to establish an emotional connection. That connection was both established and mediated by technology, in the form of the electrically amplified microphone.

\section{Pop Music and Technology}

In regards to pop music within this thesis, I am specifically referring to the modern, contemporary form of popular music that has emerged since the early 1980s, which has emphasized digital instrumentation and recording practices, high studio production values, relatively short songs (three to four minutes on average), simple compositional structures, the use of repetitive melodies, hooks and choruses, precise vocal performances, and a clearly commercially-oriented focus (particularly towards the youth or teen music listening market). Artists such as Madonna, Britney Spears, Christina Aguilera, Mariah Carey, New Kids on the Block, The Backstreet Boys, $N^{\star}$ Sync, Lady Gaga, and others, are all prime examples of the modern musical paradigm of pop music: a highly commercial, highly produced, musically and vocally precise form of popular music which emphasizes short, simple, melodic songs that are designed to appeal to a broader, more general audience (rather than a specialized subgenre or subculture).

The modern paradigm of pop music has demonstrated a close connection to technology, both in regards to the adoption of influential digital instruments as well as changes to studio production methods and techniques. For example: synthesizers played a vital role in the formation and sound of not only 
progressive-rock, but in synth-pop and new-wave during the late 1970s and early 1980s. Drum machines have provided the rhythmic foundation for pop music arrangements for decades, since they provide programmable, quantized, perfectly metronomic rhythm (as well as proving more cost-effective than a professional session drummer). And, as discussed earlier, Digital Audio Workstations (DAWs) such as Pro-Tools have revolutionized the practice of not only recording, but composing pop music (in that they allow producers to restructure and combine various takes into a completed, idealized recording). Auto-Tune's initial reception and subsequent widespread adoption within pop music can be attributed to the software's pitch correction technology, which appeals to the genre's core aesthetic values of vocal perfection, precision, and high studio production values. Because the software enables pop music producers and engineers to correct the vocal pitch of a pop musician's performance, Auto-Tune effectively complements the vocal aesthetics and conventions of modern pop music, given that the genre values vocal perfection and precision in its recordings, and was in the first instance founded partly on technological mediations of the voice.

But a question arises: why is it that other genres, which also value vocal precision, have not also adopted Auto-Tune as enthusiastically as pop music? For instance, why has Auto-Tune not received the same enthusiastic adoption within gospel music, as it has received within pop music, since gospel music also highly values skilled, precise, virtuoso vocal performances? The catalyst for pop music's enthusiasm for, and other genre's hesitation towards, Auto-Tune is due to 
pop music's other generic value: that it is (generally speaking) an avid and early adopter of new, digital music technologies. Much like the microphone, digital synthesizers, drum machines and DAWs before Auto-Tune, "[pop music] is increasingly enhanced by technology: the sound system on which commercial pop music depends has evolved from simple amplification to a creative tool which can modify tone-colour and create simultaneous harmonies ..."119 AutoTune appeals to the symbiotic relationship between pop music and digital technology, a relationship in which the music is both inspired and meditated by the technology used to produce it. While other genres find inspiration in the dynamic relationship between the arrangement of vocals and instruments (both acoustic and electric), it is pop music which is particularly fascinated with -- and accepting of -- new forms of digital music technology.

The relationship between pop music and music technology, particularly in regards to studio technology, is explored in greater detail by Timothy Warner. Warner, as a music-technologist, provides key insights into the use of technology in the production of pop music, as well as how music production technologies (such as Auto-Tune) appeal to a variety of modern pop music production values and practices. Warner argues that technology has become a core value of pop music, since the strong link between pop music and an ever-developing technology ensures a continually evolving 'sound' in pop."120 Technological

\footnotetext{
119 "Singing: 20"h Century," The New Grove Dictionary of Music and Musicians, 435.

120 Timothy Warner, Pop Music: Technology and Creativity: Trevor Hom and the Digital Revolution (Burlington, VT: Ashgate Publishing Ltd., 2003), 18.
} 
advancements, innovations, as well as changes in the application of music technology influence changes in the sound of pop music itself (the Auto-Tune Cher-effect being a prime example of how technological innovation can change the sound of pop music).

Warner also credits pop music for spearheading the music industry's migration to digital recording technologies: one of the key motivations for pop music's transition from analog to digital music technology arose through the genre's attempt to contain and/or eradicate the distortion produced by analog tape recording. As explored in Chapter II, DAWs record sound digitally with a high level of clarity and fidelity, which some

fans and producers of certain kinds of popular music had difficulty accommodating [to] this new-found clarity. Many rock musicians and producers, in particular, were reluctant to embrace the new digital technologies, as their use resulted in sonic characteristics that were radically different from, and uncharacteristic of, the established genre. ${ }^{121}$

What Warner is indirectly positing is that digital recording technology posed a challenge to the sonic aesthetic of rock music, since it produced a new sonic clarity and fidelity that was at odds with the preferred sonic character associated with rock aesthetics.

Producers of pop music records, on the other hand, were among the first to champion the new digital technologies. Consequently, it was largely pop musicians who were responsible for evolving and developing specific working practices associated with these new technologies, and these practices have now largely become established throughout the record industry. ${ }^{122}$

\footnotetext{
121 Warner, Pop Music, 22.

122 Warner, Pop Music, 22.
} 
Pop musicians (and producers) became the early champions of digital recording technology because it resolved the ongoing issue pop music had with the limitations of analog tape recording, namely that it produced an audible amount of distortion. With digital recording technology, the sonic limitations of analog recording were overcome, enabling the production of a clear, distortion free, endlessly editable recording. It is these two facets of digital recording technology, clarity and customization (or "increased flexibility and resourcefulness to the musician," according to Warner), that most appealed to early pop musicians and producers, since they allowed these musicians and producers to overcome the limitations of analog recording technology. What this insight into pop music's championing of digital recording technology offers is a reason why pop musicians and producers became early adopters of Auto-Tune, helping to establish the technology within the broader music industry and also developing a set of initial, specific working practices.

In regards to how certain DSP sounds and effects become conventionalized within the music production process, Warner (in his exploration of how modern DSPs imitate reverberation) says that

... the use of effects is often the result of musical convention rather than the depiction of some natural acoustic phenomenon. Hence, a certain process may be applied to a particular signal, in the first instance, as one way of differentiating it from other recordings. However, a successful recording of this sort will spawn many imitators and as a result may establish new conventions of signal processing. ${ }^{123}$

123 Warner, Pop Music, 24. 
Such was the case with the discovery of the Auto-Tune vocal effect in Cher's "Believe": the application of Auto-Tune to the song may have begun as a more conventional pitch correction, but the decision to place the program's settings at their extreme was a way of differentiating this use of Auto-Tune from other recordings. And, given the immense success of the effect in the song, many imitators soon appeared, each attempting to ascertain how the effect was produced so that they could apply the effect to their own projects. This process consequently established a set of conventions for the utilization of Auto-Tune within pop music.

The use of digital instrumentation also has an influence on the necessity for precise vocal tuning, particularly in pop music. David Mellor makes a point in favour of using Auto-Tune by suggesting that

... when you have a singer who is a little less than perfect, backed by electronic or digital instruments that are always exactly in tune, Auto-Tune will probably help. The comparison between the perfect tuning of the instruments against the slight approximations of the singer is often uncomfortable to listen to. Auto-Tune can make the singer as accurate as the instruments. ${ }^{124}$

This is an important insight: music that uses or relies upon digital instruments with exact, invariant tuning is more ideally suited to the implementation of AutoTune, since the Auto-Tuned voice will reflect the invariant tuning of the digital instruments. This, in part, explains why pop music and recent hip hop music are more conducive to Auto-Tune: both use digital instruments (i.e. digital

124 David Mellor, "When should you use Auto-Tune, and when should you avoid it like the plague?" RecordProducer.com, April 4, 2009, accessed February 16, 2012, http:/l www.recordproducer.com/?a=10. 
synthesizers, samplers, MIDI controllers, etc.) that produce perfect and/or stable tuning unless deliberately programmed to do otherwise. The human voice, with its natural tendency towards "approximate" tuning, can potentially present intonation problems when layered overtop of digital instrumentation. This partly suggests reasons Auto-Tune might have been applied to Katy Perry's octave jump in the chorus to "California Gurls (with Snoop Dogg)," since the approximate, relative tuning of Perry's octave jump (as heard in her live performance of the song on The Late Show with David Letterman) would have clashed with the precise, stable tuning of the song's digital instrumentation (as heard on the studio recording). The application of Auto-Tune within hip hop and pop music recordings, therefore, addresses and overcomes an aesthetic and technical issue amongst studio producers, engineers and musicians: Auto-Tune's pitch correction technology produces stable and precise tuning between the human voice and the digital instrument, since the Auto-Tuned voice is brought into intonation with the invariantly tuned digital instrumentation of both pop music and hip hop.

However, it must be pointed out that David Mellor's opinions on how, when, and under what technical circumstances Auto-Tune should be applied must also be put into context: as the Course Director of Audio Masterclass, ${ }^{125}$ and as author of another opinion piece on how vinyl is superior to digital audio,126

\footnotetext{
125 "The Audio Masterclass Music Production and Sound Engineering Online Course," Audio Masterclass, accessed February 28, 2011, http://www.audiomasterclass,com.

126 David Mellor, "It's true! Vinyl IS better than digital!!," RecordProducer.com, February 18, 2010, accessed February 16, 2012, http://www.recordproducer,com/index.php?a=92.
} 
Mellor represents and subscribes to an aesthetic of music production that predominantly prefers analog technology. As his articles on Auto-Tune and vinyl records demonstrate, Mellor still highly regards the sound and aura of analog recordings and of acoustic sound, rather than the perfect tuning of digital instruments and high fidelity of DAWs like Pro-Tools. Given all of this, and also given the polemic titles of his articles (the above quotation was taken from his article entitled "When should you use Auto-Tune, and when should you avoid it like the plague?"127), Mellor's opinions in regards to Auto-Tune should be taken with consideration for his biases.

\section{Old-School Hip Hop Vocal Aesthetics}

Unlike pop music, which managed to incorporate Auto-Tune in a relatively complementary fashion, the use of Auto-Tune in hip hop has represented a point of great internal conflict and contention. It has resulted in a schism between two factions within the hip hop community. But what, precisely, are the tenets of the old-school hip hop aesthetic which detractors of Auto-Tune cite in their dismissal of the technology? While there are a number of clear, distinct musical and stylistic values that distinguish pop music from hip hop, there are a few overlapping values shared by both genres, particularly in terms of how each genre conveys their lyrical text. Much like pop music, "the antecedents of rap lie in the various story-telling forms of popular music: talking blues, spoken

\footnotetext{
127 David Mellor, "When should you use Auto-Tune, and when should you avoid it like the plague?" RecordProducer.com, April 4, 2009, accessed February 16, 2012, http:ll unw. recordproducer.com/?a=10.
} 
passages in gospel, and call and response." ${ }^{128}$ The emulation of speech, therefore, is not only a key aspect in how lyrics are delivered in pop music, but in hip hop as well.

Rap is rhythmic talk, talk leaning toward music. Yet all speech is to some degree rhythmic, with stresses occurring at regular intervals to make sounds easier to understand (in English, for example, stresses divide sounds into equally spaced units regardless of how slow or fast the speech); and all speech is musical in that some words are pitched higher or lower than others. What's more, stress and pitch are related (in English, high-pitched words are normally stressed). Rap, then, falls somewhere between the worlds of music and talk, but sharing with both. ${ }^{129}$

Whereas pop music's vocal form is predominantly sung (but with the intent of conveying lyrics in a manner reminiscent of speech), hip hop lyrics are conveyed in a hybrid vocal form: it is simultaneously spoken and sung, delivered as rhythmic, harmonized speech coupled with underlying, repeating, sampled instrumentation. It is a vocal form that is unique within modern popular music, and is one of the key musical traits that distinguishes hip hop from other genres. But what, exactly, are the unique vocal aesthetics of old-school hip hop, and how does this aesthetic inform and/or influence critics' opinions regarding Auto-Tune?

The tenets of old-school hip hop vocal aesthetics can be understood through an analysis of the lyrical and stylistic distinctions that historically occurred between east coast and west coast hip hop over the course of the 1980s and 1990s. When both are taken into consideration, these approaches

${ }^{128}$ Roy Shuker, "Rap; Hip Hop," Key Concepts in Popular Music, (London/New York: Routledge, 1998), 247.

129 John F. Szwed, "The Real Old School," The Vibe History of Hip Hop, ed. Alan Light, (London: Plexus Publishing, 1999), 4. 
combine to form an all-encompassing vocal aesthetic framework of old-school

hip hop. Matt Diehl explores the core values, aesthetics, and distinctions of both

scenes:

Just as New York is the home of edgy independent movies and Hollywood that of slick entertainments, Cali rappers aimed to please the largest possible audience, while the jazzbo-virtuoso New York rhymers were often more interested in demonstrating skills to their peers more than anything else ... While [N.W.A.'s] ghetto tales came from the streets of South Central L.A., they were presented in exciting, cinematic narratives that played like blaxploitation action-movie blockbusters, making hard rap that much more accessible to a wider audience.

New York rap often seemed deeply insular - the tricky wordsmith pyrotechnics and cryptic references ... was music to be played on Walkmans while riding on the subway or cut up by DJ Red Alert in sweaty after-hours underground clubs. Also, much of it was interior ... as well as spiritual, frequently laden with the insider-only rhetoric of Muslim sects like the 5 Percent Nation. West Coast hip hop, in contrast, was driving music, ready-made to blare out of car windows and share with the world. ${ }^{130}$

As Diehl's above examination of east coast vs. west coast aesthetics demonstrates, both scenes practiced somewhat paradoxical approaches to hip hop: east coast rap is considered a more cerebral, jazz-influenced, lyrically inventive, competitive and underground form of hip hop, whereas west coast hip hop is typified as more crowd-pleasing, entertaining, cinematic party music (but also with a tendency towards violent representations). When combined, these two stylistic approaches nevertheless contribute to what can be considered the old-school hip hop paradigm, in which the MC's lyrics in the verses are the primary focus of the song. MCs, utilizing their half-music, half-talking hybrid vocal

\footnotetext{
130 Matt Diehl, "Pop Rap," The Vibe History of Hip Hop, ed. Alan Light, (London: Plexus Publishing, 1999), 128-129.
} 
form, represent the core of the old-school hip hop aesthetic, while the chorus is most often derived from a sample or sung by a special guest singer. Old-school hip hop, on both coasts, valued the MC's lyrical creativity and originality over any notion of conventional singing ability.

Also, not only is priority given to the MC over the instrumentation in oldschool hip hop productions, but the MCs are also bestowed with an ability and agency to articulate the socio-economic conditions and affairs of their community. As Anthony DeCurtis says, "... if you are part of that community, rap provides a reflection and an expression of your daily life, suggests that you can be part of the cultural conversation that helps shape this country, and infuses your experience with all the dignity and seriousness artistic representation imparts. ${ }^{n 131}$ The old-school hip hop vocal aesthetic espouses not only a particular half-music, half-sung, rapped vocal form, but also a particular onus on the $M C$, as the voice of a larger community, as both the articulator of, and contributor to, a larger discourse.

In contrast to this old-school aesthetic, in modern hip hop and particularly in hip-pop, Auto-Tune has enabled new artists to redefine their roles as MCs; to assert themselves as singers, pulling double duty as both $\mathrm{MC}$ and vocalist, which has in turn placed an increased emphasis on the chorus (a prominent aspect of pop music) rather than the verses (the focal point of old-school hip hop lyrics) in many recent hip-pop recordings. It is this aspect, in particular, that deeply irks the

131 Anthony DeCurtis, "Word," The Vibe History of Hip Hop, ed. Alan Light, (London: Plexus Publishing, 1999), 92. 
proponents of old-school hip hop aesthetics: by recasting the MC as both rapper and singer, the new cadre of hip hop artists have stylistically progressed away from (what they believe to be) the core aesthetic tenets of their genre. This is partly why Auto-Tune has met such resistance and dismissal from old-school hip hop proponents: the belief that it diminishes the power of the MC's words. It recontextualizes the role of the MC away from the articulator of prevailing social, political, and economic discourses within the hip hop community into a digitallyassisted, crooning pop singer, potentially nullifying the MC's political agency and impetus in the process. For the old-school guard, the use of Auto-Tune is an ethical one, since it leads to the redefinition and recontextualization of hip hop's core aesthetic values and stylistic tenets.

\section{Hip-Pop Aesthetics}

For the cadre of young, pro-Auto-Tune hip hop artists, the utilization of Auto-Tune within their music does not pose the same ethical issues as it does for old-school hip hop proponents: it instead represents the new sound of hip hop, a new musical paradigm and aesthetic which they can explore and develop. For these artists, incorporating Auto-Tune into their music is a way of being a part of the hip hop vanguard, of producing a hit single, of being recognized within the hip hop mainstream as a hot new artist with a hot new sound, and of achieving far greater commercial success within the pop music charts (as many pro-Auto-Tune 
hip-pop artists -- such as T-Pain, ${ }^{132}$ Kanye West, ${ }^{133}$ Drake, ${ }^{134}$ the Black Eyed Peas, ${ }^{135}$ and others -- have experienced). In modern hip hop, value is increasingly placed on the MC's ability to sing; the Auto-Tune Cher-effect not only enables this, but also endows the performance with a certain modern, appealing caché.

However, the use of Auto-Tune as the catalyst for hip hop's current stylistic dalliance with pop music is merely the most recent manifestation of an ongoing trend in which hip hop artists have infused pop music sensibilities into their own hip hop idiom. Matt Diehl offers a definition of hip-pop, which he refers to as "pop rap," as well as an insight into the hip hop genre's paradoxical attitude towards mass-appeal:

So what do we mean by 'pop rap' anyway? Most simply, the word pop (as in 'popular') signifies music that's reaching for the biggest conceivable audience. For many rap fans, such an approach inherently means gentrification of hip hop - yet hip hop's original intent was always about sucking the biggest possible audience into its groove. When DJs like Kool Herc cut up vinyl in Bronx parks back in the '70s and early ' 80 s, they were trying to move as big a crowd as they could. ${ }^{136}$

132 "T-Pain Album \& Song Chart History," Billboard.com, accessed March 28, 2012, http:/l uww.billboard.com/artist/t-pain/690156\#/artist/t-pain/chart-history/690156.

133 "Kanye West Album \& Song Chart History," Billboard.com, accessed March 28, 2012, http:/l www.billboard.com/artist/daft-punk/chart-history/141900\#/artist/kanye-west/chart-history/322005.

134 "Drake Album \& Song Chart History," Billboard.com, accessed March 28, 2012, http:/l www.billboard.com/artist/daft-punk/chart-history/141900\#/artist/drake/chart-history/855020.

135 "The Black Eyed Peas Album \& Song Chart History," Billboard.com, accessed March 28, 2012 , http://www.billboard.com/artist/daft-punk/chart-history/141900\#/artist/the-black-eyed-peas/charthistory/272581.

136 Diehl, “Pop Rap,” 122. 
Diehl identifies the paradoxical stance hip hop takes regarding its mass appeal: that it strives for as big a crowd as possible, while at the same time many of its proponents fear that doing so will gentrify the genre. These "rap fans" Diehl vaguely references who fear the gentrification of hip hop can be considered hip hop heads, a label used in reference to a particular type of hip hop fan who prefers a more hardcore, pure, old-school form of hip hop. These hip hop fans, since they subscribe to the old-school hip hop aesthetic, generally oppose the fusion of hip hop with other forms of music which they believe will dilute the potency of their preferred idiom of hip hop. But the fusion of hip hop with pop music elicits certain uneasiness not only amongst hip hop heads, but sometimes amongst the greater hip hop community as well:

What, then, lies at the core of pop rap's dubious status in the hip hop community? More than anything, its critics claim, such accessibility often denies the emphasis on invention that hip hop heads have been demanding since the earliest street battles ... Pop rap's relationship to ghetto realness also remains a volatile issue, in particular when hardcore fans sense rappers sanding off their rough edges for the mainstream audience. ${ }^{137}$

The primary fear amongst the greater hip hop community is that the infusion of other musical forms and practices -- particularly those of pop music -- will alter the fundamental form and practice of the genre, specifically the "emphasis on invention" (the lyrical creativity and inventiveness of the MC), a loss of "ghetto realness" (an honest connection with -- and depiction of -- the socio-economic realities of the urban, hip hop community), and the softening of one's image and

137 Diehl, “Pop Rap," 122. 
style for the sake of greater mainstream acceptance, recognition, and/or success.

However, there nevertheless exists a long legacy in hip hop where the genre has experimented with pop music practices and sensibilities, and subsequently achieved considerable mainstream success and recognition: D.J. Jazzy Jeff and the Fresh Prince's "Parents Just Don't Understand, ${ }^{{ }^{1138}}$ The Beastie Boys' "(You Gotta) Fight For Your Right (To Party),"139 Vanilla Ice's "Ice Ice Baby,"140 Marky Mark's "Good Vibrations,"141 Sir Mix-A-Lot's "Baby Got Back,"142 Salt-N-Pepa's "Push It,"143 Kris Kross' "Jump,"144 and MC Hammer's "U Can't Touch This"145 have all achieved both hip hop and pop music commercial success by blending elements of both respective genres. All of the above songs transgressed the hip hop genre's core musical aesthetics by infusing specific elements of pop music -- such as hook-heavy choruses, simple instrumentation,

138 D.J. Jazzy Jeff and the Fresh Prince, "Parents Just Don't Understand," He's the DJ, I'm the Rapper, Jive B0015XWUIQ, compact disc, 2008, originally released in 1988.

139 The Beastie Boys, "(You Gotta) Fight For Your Right (To Party)," Licensed to III, Def Jam Recordings B0000024JN, compact disc, 1995, originally released in 1986.

140 Vanilla Ice, "Ice Ice Baby," To The Extreme, EMI Music Canada B00000DRBV, compact disc, 1999 , originally released in 1990.

141 Marky Mark and the Funky Bunch, "Good Vibrations," Music For the People, Interscope Records B000026GR9, compact disc, 1991.

142 Sir Mix-A-Lot, "Baby Got Back," Playlist: The Very Best of Sir Mix-A-Lot, Rhyme Cartel/ American/Legacy B002984AR0, compact disc, 2009, originally released in 1992.

143 Salt-N-Pepa, "Push It," Hot, Cold \& Vicious, London Records B000001FHE, compact disc, 2008, originally released in 1986.

144 Kris Kross, "Jump," Totally Krossed Out, Sony BMG B0012GMYMI, compact disc, 2008, originally released in 1992.

145 MC Hammer, "U Can't Touch This," Please Hammer Don't Hurt 'Em, EMI Music Canada B000002UVD, compact disc, 1990. 
recognizable samples, (relatively) inoffensive language and subject matter, and a general accessibility -- and targeting their music towards a more mass audience, above and beyond the hip hop community.

However, with massive commercial success amongst pop music audiences, a degree of controversy arises in regards to that artist's status within the hip hop community. In regards to MC Hammer, Diehl explores how Hammer's huge commercial success entailed a number of contentious issues amongst the hip hop community:

Hammer's entree into the big time spawned rap's greatest aesthetic controversy yet. He had some unlikely boosters - rap's foremost polemicist, Chuck D, publicly admired Hammer's business acumen, which he saw as a positive example. "You're supposed to sell out!" Chuck D told Rolling Stone at the time. "If you got fifteen tapes on the shelf, your mission is to sell. You ain't giving it away. So I can't get mad at Hammer for doing what he's got to do." Many, though, were aggravated by Hammer's pretested, predigested hits, as well as his emphasis on dancing and feel-good entertainment over rhyme skills. ${ }^{146}$

MC Hammer's musical idiom, which prioritized pop music sensibilities such as dancing and feel-good entertainment over core hip hop aesthetics such as rhyme skills, irked many within the hip hop community, since his music adhered to neither the east coast aesthetic of inventive, cryptic lyricism, nor the west coast's entertaining yet realistic depiction of socio-economic hardships.

But what, precisely, is the process by which established, underground artists begin to cross over into hip-pop territory, and how are musical and stylistic elements of pop music brought into -- and fused with -- hip hop musical practice?

146 Diehl, “Pop Rap,” 128. 
Again, Diehl offers an anecdotal example of how a distinguished hip hop artist negotiates the musical and stylistic boundaries between pop music and hip hop within their music:

When Nas busted out with his debut album, 1994's IIImatic, he was hailed as a neo-realist ghetto poet, a welcome return to rap's lyrical invention roots; as a result, the album crawled to gold status. Yet when Nas released his sophomore album it Was Written in 1996, his image and sound had undergone subtle changes that produced not-so-subtle results. Nas's around-theway gear had been exchanged for fly suits that wouldn't have been out of place on Robert De Niro in Casino, and cooking female choruses proliferated ... in "Street Dreams" he even utilized pop rap's sure-shot ploy of lifting hooks from rock hits, in this case subverting the infectious chorus from the Eurythmics' 1984 synth-pop hit "Sweet Dreams (are Made of This)." Nas's lyrical skills remained in effect, however, and the symbolism was as ghetto as ever -- these were "Street Dreams," after all, not "Sweet Dreams" -- but the overall effect was that much more palatable to a wider audience. The payoff of Nas's overhaul was seen in the multiplatinum sales of It Was Written. ${ }^{147}$

By crafting a more sophisticated image (i.e. De Niro-inspired suits), heightened recording/production values, utilizing pop rap ploys like lifting hooks from popular music hits, and appealing to a wider audience with more palatable hip hop material, Nas' sophomore album flirts with mainstream, pop music values, yet manages to maintain his initial hip hop credentials through his lyrical skills and use of ghetto symbolism. Other hip hop artists and groups have either attempted and/or managed to musically and stylistically balance hip hop aesthetics and credentials with pop music forms, sensibilities, recognition and success. The

147 Diehl, “Pop Rap," 131. 
Fugees, for instance, managed to achieve a hybrid hip hop and pop music formula on their breakthrough album The Score:148

Indeed, the Fugees often followed pop rap's hit-making formula to a T: As with Hammer's "Have You Seen Her," their versions of Roberta Flack's "Killing Me Sottly" and Bob Marley's "No Woman, No Cry" were straight-up covers with amped-up beats. However, the Fugees' remakes didn't rankle hip hop purists as Hammer's did -- they felt more like organic evolutions out of their own influences than desperate chart stabs; they were also surrounded by idiosyncratic hip hop grooves and shouts of Haitian pride..$^{149}$

What Nas, The Fugees, MC Hammer, and other artists establish is a continuum, an ongoing practice within hip hop where artists experiment with various pop music sensibilities, resulting in both commercial success amongst a larger popular music audience and conversely a re-evaluation of the artist's status and/ or credibility amongst a more ideologically driven hip hop audience.

\section{Conclusion}

Auto-Tune, within the context of hip-pop, acts as a catalyst for not just the transference of musical style, sound and sensibility between hip hop and pop music, but the transgression of established, old-school hip hop aesthetics and genre conventions. Such transgressions, as Simon Frith outlines, are frequently used to forge a new set of conventions and musical practices, which, in time, contribute to the formal establishment of new subgenres.

... One way in which genres work in day-to-day terms is in a deliberate process of rule testing and bending. As Charles Hamm has argued, popular musicians 'work within a tradition that allows

\footnotetext{
148 The Fugees, The Score, Sony Music Canada B000002B5L, compact disc, 1996.

149 Diehl, "Pop Rap," 131.
} 
and even demands flexibility and creativity in shaping a piece. Genre is not determined by the form or style of a text itself but by the audience's perception of its style and meaning, defined most importantly at the moment of performance. Performers can thus shape, reinforce or even change genre.' It is out of such 'transgressive' performances that genre histories are written: old genres 'fail' when their rules and rituals come to seem silly and restrictive; new genres are born as the transgressions become systematic. ${ }^{150}$

Because of the systematic transgressions conducted by artists such as T-Pain, Lil Wayne, Drake, Kanye West, and others, their use of Auto-Tune has essentially rewritten the rules and rituals upon which the old-school hip hop aesthetic is founded. The introduction of Auto-Tune into hip hop represents not only the development of a new idiom of hip hop, but a crossover project between hip hop and pop music; a stylistic transgression between the musical form of two genres, as well as the transference of a variety of musical practices and preferences. Auto-Tune represents a vehicle by which hip hop artists are able to transgress and/or break the boundaries of old-school hip hop musical traditions, values and aesthetics, to form their own rules and rituals regarding musical practice and convention, and to import pop music vocal techniques and sensibilities, as well as the compositional structures of pop music (specifically the genre's emphasis on the chorus). These systematic transgressions within hip hop have consequently led to the mainstream reemergence of hip-pop as a subgenre, which positively affirms and aesthetically values Auto-Tune. The restrictions of the old-school paradigm towards technologies like Auto-Tune have likely precipitated and/or influenced new hip hop artists to transgress these values, and

150 Simon Frith, Performing Rites (Cambridge: Harvard University Press, 1996), 93-94. 
consequently to form their own rules, rituals, and practices endorsing the use of Auto-Tune.

What this chapter has attempted to highlight are some of the aesthetic values associated with three specific (sub)genres -- pop music, hip hop, and hippop -- in an effort to show how and why artists, producers, and fans within these genres tend to react to Auto-Tune in accordance with the aesthetic code of their respective genre. Each genre is defined according to a distinct set of aesthetic criteria and musical parameters. These parameters are indicative of each genre, but they are not static: genres are fluid, in that they change and adapt to the ways musicians practice a particular form of music; responding to, challenging, and even transgressing the aesthetic criteria of their respective genre. As Roy Shuker stipulates:

... no style is totally independent of those that have preceded it, and musicians borrow elements from existing styles and incorporate them into new forms ... Further, many performers can fit under more than one classification, or shift between and across genres during their careers. There is also considerable genre-bending: subverting or playing with the conventions of existing musical genres, or adopting an ironic distance from those same conventions. This process is strongly present in hybrid genres, where different styles inform and engage with each other (e.g. jazz rock). ${ }^{151}$

Such is the case for many modern musicians, whether they be pop musicians or hip hop musicians, since the increased frequency of crossover projects (especially between hip hop and pop music) has led to various forms of

151 Roy Shuker, "Genre; meta genres; subgenres," Key Concepts in Popular Music, (London/New York: Routledge, 1998), 146. 
transgression and transference between the genres, whether they be stylistic, technical, practical, or aesthetic. 


\section{V - Theory}

While Chapter IV highlighted the various ways in which musicians and listeners react to the adoption of Auto-Tune within their respective genre in accordance with the aesthetic framework and values of that particular genre, and Chapter III explored a range of examples of how Auto-Tune is applied to music produced within these specific genres, this chapter shall draw upon various theoretical models in order to examine what symbolic effect Auto-Tune has upon the music it is applied to, as well as exploring how Auto-Tune can be understood according to those theoretical models.

In this chapter I will draw upon the work of Naomi Cumming, who applies a Peircean model of semiotics to music in order to decipher the various modes and degrees of musical signification, and to explore what potential meanings these significations are best able to convey and/or achieve. By analyzing AutoTune from the perspective of Peircean semiosis, it will be possible to discern the virtual persona Auto-Tune generates, how that persona functions as an agent of musical expressivity, and how the virtual persona effects the initial, subjective identity of the singer. From there, 1 shall conduct a brief semiotic analysis -informed by the work of Roland Barthes -- on the grain of the Auto-Tuned voice, with the intention of understanding what semiotic effect the Auto-Tune effect has upon the grain of the singer's voice. Thirdly, I will introduce the notion of the hyperreal, as explored by Jean Baudrillard. Because the Auto-Tune software is able to generate a hyperreal representation of a singer's voice, above and 
beyond the physiological capabilities, idiosyncrasies and/or limitations of the human voice, a number of postmodern issues and concerns are raised (as a reading of Baudrillard's postmodern philosophy will show). Finally, I shall draw upon the notion of schizophonia -- the disassociation of musical sound from its source -- in order to explore the sensation of, and methods by which, Auto-Tune disassociates the singer's voice from his or her body.

\section{Naomi Cumming and The Sonic Self}

In her book The Sonic Self, Naomi Cumming explores how musical meaning can be both expressed and interpreted. She writes from -- and is informed by -- the experience and expertise of being both a trained violinist and a scholar of semiotics and western philosophy. Cumming argues that the sonic self is 'the individual 'subject-position,' or personal point of view, within a broader social and semiotic framework." ${ }^{152}$ Since the sonic self, and musical interpretation itself, can be highly subjective, Cumming in-effect accepts subjectivity as an integral component in the process of musical interpretation. As well, Cumming values the act of performing in her writing: "to her, music is not only sound, but the experience of making the sound and the experience of the potential elusiveness of communicative sound. She focusses on timbre, an

\footnotetext{
152 Naomi Cumming, The Sonic Self: Musical Subjectivity and Signification (Bloomington: Indiana University Press, 2000), 290.
} 
element of music inextricable from performance, instead of elements such as pitch, rhythm or form that can be frozen as objects. ${ }^{n 153}$

But what exactly is Peircean semiosis, and how can it be applied to an examination of musical signification? Unlike Saussurean semiotics, in which the signifier and signified are arbitrarily correlated into a bilateral sign through the conventions of language (via a network of connected speakers), Cumming subscribes to the Peircean school of semiosis, in which the process of signification is expanded from a bilateral into a triadic process. In the Peircean model, the sign is broken into three distinct, essential elements:

1. the Representamen: similar to the Saussurean 'signifier'; the source or indicator of signification.

2. the Object: similar to the Saussurean 'signified'; "that which the sign/ Representamen stands for," ${ }^{\text {"154 }}$ which can be either an immediate object ("the object as it is represented by the sign"155) or a dynamic object ("the object independent of the sign which leads to the production of the sign"156).

3. the Interpretant. the thought, image or meaning invoked in the mind of the interpreter when exposed to the Representamen, and the entity which

\footnotetext{
153 Margaret Walker, "Naomi Cumming. The Sonic Self: Musical Subjectivity and Signification," Discourses in Music: Volume 4 Number 1 (Fall 2002), accessed February 17, 2012, http:ll wmw. discourses,ca/v4n1a5.html.

154 Paul Cobley \& Litza Jansz, ed. Richard Appignanesi, Introducing Semiotics (Thriplow, Royston, UK: Totem Books, 1999), 22.

155 Cobley and Jansz, Introducing Semiotics, 22.

156 Cobley and Jansz, Introducing Semiotics, 22.
} 
mediates the process through which a Representamen is able to signify an Object.

Unlike the Saussurean semiotic model in which the process of signification is a bilateral process in which the signifier is arbitrarily coupled to or associated with a signified, the Peircean model of semiotics allows for far more flexibility in both the interpretation and examination of signification since Peirce's theory expands the triadic sign into three further categories of phenomena. Peirce proposes that all three components of the sign (the Representamen, Object, and Interpretant) may be related to any of three existential and operational modalities, which he calls Firstness, Secondness, and Thirdness. Peirce describes Firstness as a possibility which vaguely conveys or signifies a particular quality, characteristic or feeling (like a colour or musical note, for example). Secondness is the realm of facts which arise through the encounter with obdurant entities (usually framed as material), while Thirdness is the realm of general laws, abstract relations, and convention. "Transposed onto Peirce's sign triad, the categories result in the following: the Sign or Representamen is a First; the Object is a Second; the Interpretant is a Third. ${ }^{n 157}$ Fully realized, the Peircean model of semiosis thus consists of sign types, derived by ordering the three elements of the Peircean sign (Representamen, Object, and Interpretant), according to the three categories of existence or operation (Firstness, Secondness, and Thirdness). Table 1, below, demonstrates these nine key modes of sign-generation:

157 Cobley and Jansz, Introducing Semiotics, 28. 


\begin{tabular}{|c|c|c|c|}
\hline \multicolumn{2}{|c|}{ Table 1 } & Sinsign & Legisign \\
\hline Qualisign & Index & Symbol \\
\hline Icon & & Argument \\
\hline Rheme & Dicent & Argume \\
\hline
\end{tabular}

Peircean semiosis espouses three levels of object signification: the first trichotomy -- Firstness -- addresses the quality of the Representamen (rather than what it indicates), Secondness pertains to the operation or function of the sign (through either resemblance, causation, or convention), while Thirdness is about what sort of Object is being signified. What all these categories, elements and orders enable is a greater degree of specificity in not only identifying, but evaluating, examining, and understanding the various components of signification in a way that is not fostered or enabled within Saussurean semiotics.

How, then, can a Peircean system of semiotics be applied to music? Cumming does so by stipulating that, in general, musical signification occurs and functions primarily within the realms of Firstness and Thirdness. Musical signification, particularly non-lyrical instrumental music, does not adequately signify Secondness because it is extremely difficult to establish how non-lyrical instrumental music signifies, relates to, or clearly connects to an Object. 
Significations found in non-texted instrumental music may be taken, in accordance with this scheme, as belonging to a class whose objects are not 'actual' but more of a 'May-be or a 'Wouldbe.' They belong, that is, to a class where both signs and their objects display more of the characteristic of Peirce's 'first' and 'third' categories than they do of his 'second' one. Certain qualities seem to have an 'immediate' content (in the phenomenological sense), though it is interpreted (firsts). Conditionally established stylistic codes (thirds) also present contents known to those familiar with the style. Possibilities of content, understood either as seeming immediacies, or as conventions, take precedence over reference to actualities which may be stated as 'fact.'158

In this connection Margaret Walker addresses what she takes to be Cumming's side-stepping of the issue of how music relates to Peirce's notions of Secondness:

Quoting Peirce, she takes his description of each sign as 'a Maybe' (firstness), 'an Actual' (secondness) or 'a Wouldbe' (thirdness) and classifies musical significations as displaying 'more of the characteristics of Peirce's 'first' and 'third' categories than they do of his 'second' one' (Cumming 2000, 79). She deals with the problem of secondness, therefore, simply by saying that it is not applicable to musical signification. ${ }^{159}$

While Walker considers Cumming's downplaying of the relationship between musical signification and Secondness to be a fault, Walker is mistaken in saying that Cumming considers Secondness as "not applicable to musical signification." On the contrary, Cumming argues that the immediate Object (the object of thought), as opposed to the more commonly utilized dynamic Object (the existent object), is more suited to a Peircean semiosis of music. If the immediate Object, rather than the dynamic Object, is the predominant form of the Object involved in

\footnotetext{
158 Cumming, The Sonic Self, 79-80.

159 Margaret Walker, "Naomi Cumming."
} 
musical signification, then the Object to which a musical Representamen is referring to can be more abstract; a less dynamic, factual Object than in other forms or modes of signification. In this regard, that the immediate Object (as the "possibility of content") is the more commonly utilized form of Secondness within musical signification, it is not so much the case that Secondness is "not applicable to musical signification," rather that it is less applicable to musical signification (according to Cumming). Also, had Cumming dedicated herself to an examination of representamena and interpretants in musical signification, then Secondness would certainly have become a more crucial component to her examination. Having said that, Cumming's main focus in The Sonic Self is not an exploration of Secondness in musical signification, but rather an exploration of what kind of Objects music generally signifies.

Cumming argues throughout The Sonic Self that the Peircean model of semiotics is well suited to a semiotics of music, since it provides increased flexibility in determining not only what musical elements are the components of the sign (the Representamen, Object and Interpretant), but also the degree to which a musical sign signifies or corresponds to a particular sort of Object or Interpretant. What Cumming's The Sonic Self explores are the ways in which musical performances, techniques, sounds, timbres, and arrangements can be understood as signs, which can be understood in various ways, according to a variety of signification protocols as described by Peirce. 
What, then, is to be considered the source or mechanism of music's capacity for expressivity? This is a question that underpins much of Cumming's seventh chapter, in which she explores "how could instrumental music be capable of expressing emotions, when most emotional states are related to some context of activity, which cannot be musically observed. ${ }^{n} 160$ Cumming explores in what capacity instrumental music can be heard as expressing complex emotional signs -- such as hope, desire, grief, joy, pathos, etc., -- within instrumental music itself apart from an examination of the physical act of producing said music. Cumming begins by refuting Stephen Davies'161 account that "a listener knows how expressive behaviours look and translates that recognition into a way of hearing movement in sound" ${ }^{162}$ [emphasis Cumming's] by asserting that emphasizing the appearance of expressive behaviours in music is fundamentally "at odds with the experience of music as an acoustic medium, in which movements through virtual space are heard (and sometimes 'felt' - in body or mind), not necessarily seen."163 Cumming opposes Davies' view of musical meaning and interpretation based (figuratively) upon visual comprehension, since Cumming's goal is to explore both the expressive and signifying capacity of instrumental music as interpreted aurally, rather than sonically. She thus concludes that a different approach is essential in order to recognize the

\footnotetext{
160 Cumming, The Sonic Self, 197.

161 Stephen Davies, Musical Meaning and Expression (Ithaca: Cornell University Press, 1994).

162 Cumming, The Sonic Self, 201.

163 Cumming, The Sonic Self, 201.
} 
expression of more subtle and/or abstract emotional states within instrumental music.

Cumming thus draws upon Jerrold Levinson's notion of a virtual persona, "a subjective character heard as embodied in the music, as the carrier of an expressive impetus or intent." ${ }^{164}$ Although Levinson fails to elaborate in greater detail precisely how such a virtual, musical persona might be formed, he does provide a formal, logical definition for said musical persona:

$P$ expresses (or is expressive of) $A$ iff [if and only if] $P$ is most readily and aptly heard by the appropriate reference class of listeners as (or as if it were) a sui generis personal expression of A by some (imaginatively indeterminate) individual. ${ }^{165}$

Cumming, as quickly as she draws upon Levinson, critiques his logical definition, particularly in regards to the inclusion of the personal and individual as subjective paradigms by which musical expression can be discerned and/or interpreted. According to Cumming, by not only incorporating the individual into the equation, but in parenthetically distinguishing the individual as an "imaginatively indeterminate" individual, Levinson has presumed that the agency of musical expression is not only rooted in humanity, but that other forms of musical expression -- which are not explained according to the paradigm of the individual -- are excluded from such examination. Cumming disagrees with this assumption, arguing that a virtual persona -- rather than a subjectively indeterminate individual -- provides a means for the examination of the agency of

164 Cumming, The Sonic Self, 202.

165 Cumming, The Sonic Self, 202 (from Levinson, 1990, p. 338). 
musical expressivity over and above human subjectivity. Nevertheless, Cumming continues to use Levinson's logical definition as the basis for her own model of persona: as a form of musical agency within instrumental music which expresses affective states and signs. This musical agency, unlike in Levinson's definition, does not necessarily have to be considered personal or individual, according to Cumming; since persona is a virtual agent, it can therefore express musical gestures and signs through an agency not rooted in human subjectivity.

Does the 'individual' concerned need always to suggest humanoid characteristics, in order to be expressive of affective states typically possessed by people, or could it not perhaps be extended to any kind of virtual agency? ... If apparent agency may be heard -- irrespective of whether it seems to be a humanly personal utterance or not -- would this not throw into question also the confidence with which anyone could say that the expression is of 'A' (where ' $A$ ' is presumed to stand for any familiar expressive class)? An agency which is compelling, but deviates from the apparently personal, could be 'expressive' of states that are somehow recognized, yet also new and interesting. ${ }^{166}$

In Cumming's model of agency in musical expression, the virtual persona is one which expresses subtle and/or complex musical signs and states, but is not limited to or beholden to the subjectivity of the personal. Cumming's model of persona is noticeably postmodern in that it incorporates and considers a nonpersonal, non-human form of agency in musical expression.

In regards to Auto-Tune, its pitch correction and (even more so) its vocal effect can be considered a persona: a virtual character by which musical signs can be expressed, and whose agency is neither physical, personal, nor linguistic.

166 Cumming, The Sonic Self, 203. 
The Auto-Tuned voice is "a subjective character heard as embodied in the music"167; a virtual persona which acts as "the carrier of an expressive impetus or intent." 168 In the case of hip hop artists such as T-Pain, Kanye West and Drake (who uses Auto-Tune so extensively in his music that the vocal effect has become Drake's de facto vocal persona), their expressive impetus and possible intent is to recontextualize and transform not only their voices but their identities and roles as hip hop MCs into soulful singers (or crooners). In Chapter III, this Auto-Tune-as-virtual-persona dynamic was more expressly outlined in the examination of R\&B singer Mary J Blige's use of Auto-Tune on her single "The One." Blige explicitly said that using Auto-Tune in the song allowed her to create, assume, and experiment with her Auto-Tuned alter-ego "Crazy Mary." Blige's use of Auto-Tune was precisely in order to generate a virtual persona, one which established a subjective character (i.e. "Crazy Mary") and acted as a carrier of an expressive impetus or intent. As Blige remarked, Auto-Tune allowed "Crazy Mary' to get her shine on. She couldn't get her shine with my regular voice. I used the effect. ${ }^{n} 169$ In pop music vocalists, the implementation of Auto-Tune generates a virtual persona which can be utilized to express the creativity of the musician, as well as achieve the vocal setting of precise, pitch-perfect vocal performances. By augmenting the personal voice of the singer, Auto-Tune demonstrates a vocal agency which is capable of expressing affective states that

${ }^{167}$ Cumming, The Sonic Self, 202.

168 Cumming, The Sonic Self, 202.

${ }^{109}$ Shaheem Reid, "Mary J. Blige Defends Auto-Tune: 'To Each His Own'," MTV.com, June 11, 2009, accessed February 16, 2012, http:/www.mtv.com/news/articles/1613803/mary-j-bligedefends-autotune.jhtml. 
are recognizable, "yet also new and interesting." ${ }^{170}$ As Cumming points out below, the virtual persona -- as a subjective, disembodied carrier of expressive impetus or intent -- could potentially be stretched and recontextualized through (digital) music-technology:

... the very potential to hear a melodic incipit as 'gestural' ensures that the virtual 'body' can be enhanced or stretched, extended in its apparent capacities, or attenuated in its expressiveness, by the subjection of melodic incipits heard as 'gestural' to various kinds of manipulation in tonal space and time. (It is not inconceivable that a musical 'body' should be 'spaghettified,' through the electronic extension of a gestural incipit - like a body imagined by cosmic scientists to be stretched beyond its limits on entering a black hole). ${ }^{171}$

By being considered a virtual persona, the use of Auto-Tune by a vocalist essentially creates a musical character that is super-human, one whose body can be enhanced and stretched, whose expressive agency can be extended, attenuated, or manipulated through various virtual modifications to the singer's voice. Auto-Tune, in being considered a virtual persona, presents a subjective character which supplants the initial subjective character generated by the singer's voice and/or vocals. This super-ceding of the singer's initial subjective character by the Auto-Tuned virtual persona will come into play later in this chapter when I explore Jean Baudrillard's theories regarding hyperreality and simulation, as well as the notion of schizophonia (as raised by Jonathan Sterne). But first I shall here draw upon Roland Barthes' seminal text 'The Grain of the Voice" in order to conduct an examination of Auto-Tune's qualisign attributes,

\footnotetext{
170 Cumming, The Sonic Self, 203.

171 Cumming, The Sonic Self, 204.
} 
with the intention of understanding precisely what grain Auto-Tune produces, and how that grain modifies, interacts with, and/or supplants the grain of the singer's voice.

\section{Roland Barthes and The Grain of the Voice}

Whereas Cumming focuses primarily on the processes of signification, agency, and expressivity within music, Roland Barthes instead addresses the role of language in both the aesthetic criticism and interpretation of musical meaning. In this text, Barthes posits an alternative criteria by which musical criticism and interpretation may be conducted, one in which not only the body, but what Barthes calls the grain produced by the musician's body is examined and evaluated for meaning. Barthes defines the grain as "the materiality of the body speaking its mother tongue, ${ }^{1172}$ or, put another way, "the grain is the body in the voice as it sings, the hand as it writes, the limb as it performs." ${ }^{173}$ For Barthes, an examination of the degree of influence the body impresses upon a musical performance presents a new mode or approach to musical criticism, one which enables a critical examination and discussion regarding non-linguistic meanings. According to Barthes,

The grain of the voice is not - or is not merely - its timbre; the signifiance it opens cannot better be defined, indeed, than by the very friction between the music and something else, which something else is the particular language (and nowise the message). The song must speak, must write - for what is

\footnotetext{
172 Roland Barthes, "The Grain of the Voice," Image-Music-Text, trans. Stephen Heath (New York: Hill and Wang, 1977), 182.

173 Barthes, "The Grain of the Voice," 189.
} 
produced at the level of the geno-song is finally writing. ${ }^{174}$ [emphasis Barthes']

Non-linguistic meaning(s) can be found, according to Barthes, in the friction between music (as utterance) and language, rather than exclusively in the timbre of the singer's voice. Barthes' and Cumming's assertions regarding the capacity of language (or timbre) in providing an indication of music's meaning is indicative of their respective intellectual affiliations; Barthes of Saussurean semiotics and Cumming of Peircean semiosis. The former prioritizes the role of language in the interpretation of meaning, the latter prioritizes the dynamic relationship between the Representamen, Object, Interpretant, and the various orders of signification they entail. Given that my concern and focus on Auto-Tune within this thesis has thus far been geared towards what Cumming refers to as the "non-texted instrumental," rather than the texted, lyrical aspects of Auto-Tune, I believe that a consideration of the timbre of the Auto-Tuned voice -- rather than the role of language within Auto-Tuned lyrics -- will be best suited to the task of understanding precisely what grain Auto-Tune produces and how that grain impacts the grain of the singer's voice. As well, my primary focus in this section is not to conduct a semiotic analysis of the grain of any particular singer's voice, but to examine and distinguish any grain produced by the Auto-Tune software when applied to a given singer's voice.

In order to establish the grain of Auto-Tune, it must first be separated into its two distinct semiotic signs: the clandestine pitch correction, and the overt

174 Barthes, "The Grain of the Voice," 185. 
vocal effect. As mentioned in previous chapters, Auto-Tune is capable of clandestinely altering the pitch of a singer's voice. In these instances, a singer's vocals can be pitch corrected (i.e. shifting the pitch of the singer's voice from outof-tune to in-tune), and/or stabilized (i.e. rendered invariant) without the listener being aware that such corrections or modifications have occurred, since the pitch correction process leaves little audible trace of its application. Also, since the application of Auto-Tune's pitch correction to a singer's vocals is clandestine, this ensures that both the initial timbre and the grain of the singer's voice remain largely unmodified. However, there are instances where the grain of the AutoTune pitch correction can be discerned. In such instances, the grain of AutoTune's pitch correction is often a faint, vaguely nasal, synthesized drone, produced via the synthesis of vocal timbre with the software's quantized, DSP algorithms, which changes but does not entirely overshadow the grain of the singer's voice.

With regard to Auto-Tune's overt vocal effect, the grain of what is called the Cher-effect is quite different and distinct from the covert pitch correction's grain. What, then, can be said about the unique grain of the Auto-Tune vocal effect? First, the grain of the Cher-effect is inherently non-biological: when placed at the program's most extreme settings, the Auto-Tune software produces a wild, exaggerated, heavily quantized digital flutter, akin to a rapidly fluctuating digital glitch. The Cher-effect's grain is, therefore, derived digitally, rather than physiologically. This is a crucial element, since Barthes places great emphasis on the impact that a singer's physiology has upon not only their performance but the 
production of a vocal grain. With the Auto-Tune vocal effect, the vocal grain which is audible is one that is not physiologically, but digitally produced. Bodily organs, such as a singer's lungs, tongue, glottis, teeth, mucous membranes, nose, nasal cavities, etc., are no longer (as) integral or consequential in the production of this particular, unique vocal grain. Second, the heavily quantized, digitized grain produced by the Auto-Tune software not only fuses with but supplants the grain of the singer's voice. The Auto-Tune vocal effect is, in essence, a form of vocal resynthesis: a fusion of the singer's voice (and grain) with digital software calibrated to produce rapid and exaggerated vocal effects, which in-turn overrides the physiologically produced vocal grain with the quantized, digital grain produced via the software. The Auto-Tuned vocal performance is one where the singer's voice is not only digitally manipulated and effected, but is also separated from his or her indicative vocal grain.

In both instances, the grain of Auto-Tune is never produced through a human body; it bears no traces or influence of the singer's organs or unique physiology in the production of a vocal grain. Rather, the grain of Auto-Tune -both the pitch correction and the overt effect -- are grains produced digitally, through the program's DSP quantization process. In essence, the body has been removed from the process, reconstituted by the program (which uses the recording of the singer's voice as its source material). The consequence of AutoTune's tendency to generate a virtual vocal persona which supplants the physiological grain of the singer's voice with a synthetic, digitally produced vocal grain is two-fold. First, it produces a hyperreal representation of the human voice 
and professional singer. Second, the creation of a vocal grain separate from the singer's unique vocal timbre and grain produces a sensation Jonathan Sterne cites as schizophonia, the separation of sound from its source (in this case, the separation of vocals from the vocalist's body).

\section{Jean Baudrillard and The Hyperreal}

In his groundbreaking text Simulacra and Simulation, Baudrillard posits that within modern society, the pervasiveness and ubiquity of symbols and signs leads to the replacement -- rather than representation -- of reality, and that postmodern society in particular is comprised of -- and reliant upon -- these symbols to such a degree that they begin to replace the (real) world. Baudrillard considers these symbols (which he calls simulacra) to be a simulation of reality, as a stand-in for a (real) reality which has been replaced by simulation to such an extent that reality is no longer distinguishable from hyperreality.

The real is produced from miniaturized cells, matrices, and memory banks, models of control - and it can be reproduced an indefinite number of times from these. It no longer needs to be rational, because it no longer measures itself against either an ideal or negative instance. It is no longer anything but operational. In fact, it is no longer really the real, because no imaginary envelops it anymore. It is a hyperreal, produced from a radiating synthesis of combinatory models in a hyperspace without atmosphere. ${ }^{175}$

The insidious condition which Baudrillard posits and explores is that we as a postmodern society have begun to prefer and seek the simulation of hyperreality

\footnotetext{
175 Jean Baudrillard, Simulacra and Simulation, trans. Sheila Faria Glaser (University of Michigan Press, 1994), 2.
} 
to a reality not dominated or governed by symbols and signs (which seek to enhance or mediate our perception and experience of the world and reality).

It is no longer a question of imitation, nor duplication, nor even parody. It is a question of substituting the signs of the real for the real, that is to say of an operation of deterring every real process via its operational double, a programmatic, metastable, perfectly descriptive machine that offers all the signs of the real and shortcircuits all its vicissitudes. ${ }^{176}$

Hyperreality, therefore, is an artificial representation -- or simulation (to use Baudrillard's terminology) -- of reality, one which is dominated by simulations and simulacra to such an extent that we begin to prefer it to an un-simulated reality.

Baudrillard posits four orders of simulacrum, degrees by which simulacra extend and function, from representation ("the principle of the equivalence of the sign and of the real" ${ }^{477}$ ) to simulation ("the utopia of the principle of equivalence $\left.{ }^{4178}\right)$. Those orders consist of:

1) the reflection of a profound reality (i.e. the "good," close reflection of a real reality),

2) masking and denaturing a profound reality (i.e. an "evil" interpretation which distorts reality),

3) masking the absence of a profound reality (i.e. a "sorcerous," lost notion of reality), and

4) the lack of relation to any reality at all; a pure simulacrum (i.e. a simulacrum that has come to either represent a reality which does not exist, or represents

\footnotetext{
176 Baudrillard, Simulacra and Simulation, 2.

177 Baudrillard, Simulacra and Simulation, 6.

178 Baudrillard, Simulacra and Simulation, 6.
} 
nothing other than what it itself represents; "no longer of the order of appearances, but of simulation"179).

From this theoretical paradigm Auto-Tune can be analyzed and understood in regards to its symbolic effect upon the singer's voice.

As explored above, the grain of Auto-Tune is one that is produced through the software's DSP and quantization protocols; a distinct, digitally derived vocal grain. This alternative grain production process can itself be considered a form of simulation, since the process of producing a vocal grain through the unique physiology of a singer's bodily organs is instead simulated through Auto-Tune's DSP protocols. In the case of Auto-Tune's covert pitch correction capabilities, the simulation of the singer's vocal grain is a very close reflection of the singer's initial grain, since the pitch correction process manages to fuse and synthesize with the singer's vocal timbre to such a degree as to be clandestine, nearly imperceptible. In this instance, the distinction between reality (a singer singing through their body) and hyperreality (a singer's vocals as a synthesis of physiology and digital software) is significant: Auto-Tune's pitch correction capabilities, since they not only bring a singer's vocal performance back in-tune and pitch but also do so clandestinely, present a circumstance where the hyperreality of the pitch-perfect, Auto-Tuned vocalist is not only largely undetectable but also preferred to the real, unedited vocal performance. As has been explored earlier, one of the fundamental reasons why Auto-Tune was so swiftly and enthusiastically incorporated into the pop music genre was because

179 Baudrillard, Simulacra and Simulation, 6. 
of its ability to produce a vocal performance which achieved a standard of vocal precision and perfection that is highly valued according to the genre's aesthetic principles. One could argue that since Auto-Tune now enables such precise and idealized vocal performances to be more readily (and cost-effectively) realized, those performances have generally become preferred over the unedited pop music vocal performance within the genre at large; that the Auto-Tuned, pitch corrected hyperreal vocal performance is preferred to the unedited, real vocal performance. In this instance, the small, barely distinguishable distinction between pop-vocal reality and hyperreality has contributed to the preference for the hyperreal over the real pop-vocal performance.

On the other hand, the distinction between reality and hyperreality is far more exaggerated and apparent with Auto-Tune's overt vocal effect: because the Cher-effect produces such an exaggerated, heavily quantized vocal effect, the distinction between reality and hyperreality is abundantly clear and perceptible. Nonetheless, the Cher-effect represents a second order simulacra, "a masking and denaturing of profound reality, in that it presents a distorted interpretation of reality, one where the grain of the singer's voice is supplanted by a heavily quantized, wildly exaggerated vocal effect. In this instance, hyperreality tends to replace rather than to represent reality, but the distinction between the two remains perceptible.

Also, when taking into consideration the technical capabilities of Antares' Auto-Tune suite itself, other aspects and features of the software can be 
considered hyperreal. In particular, the THROAT Evo Physical Modeling Vocal Designer ${ }^{180}$ allows musicians and producers to not only edit the vocal delivery of a recording based on a digital visualization of the human throat, but also enables the producer to create a vocal dynamic that surpasses the capability and physiological limit of the human throat. While Auto-Tune's overt vocal effect affects the timbre and grain of the vocalist, the voice being edited essentially remains a human voice, governed by the boundaries of the human anatomy. However, with the THROAT Evo Physical Modeling Vocal Designer, musicians and producers now have the capacity to take a recorded vocal performance, and -- using DSP and a visualization of the singer's vocal cords -- edit that recorded voice into a hyperreal voice, one unattainable according to the human anatomy. As described by Antares Audio Technologies on the vocal tool's product page, "the range of the controls also allows the creation of vocal tract models well beyond the limits of physical human anatomy, offering the possibility of vocal characteristics that are simply unattainable by any other means." 181

The outcome of this ability is the exploration of a new realm of DSP vocal effects possibilities: with the combination of DSP technology and the creative drive to push both the technology and the aesthetics of vocality to their limits, the possibility to modify and/or manipulate a singer's voice into a hyperreal representation of their physiological voice is presented. Herein lies one major

\footnotetext{
180 "AVOX Evo Antares Vocal Toolkit," Antares Audio Technologies, accessed February 17, 2012, http:/www.antarestech.com/products/avox-evo.shtml\#throat.

181 "AVOX Evo Antares Vocal Toolkit," Antares Audio Technologies, accessed January 18, 2012, http:/hww.antarestech.com/products/avox-evo.shtmlithroat.
} 
concern some opponents of Auto-Tune have with regard to the future of this technology. While Auto-Tune is conventionally used to touch-up, pitch correct, and/or Cher-effect a singer's recorded vocal performance, the singer's voice essentially remains their voice (however overtly and/or exaggeratedly effected it may be). However, with the advancement and application of DSP technology, producers now have the capability to craft or manipulate the human voice into something beyond the physiological capability of the human anatomy; to create a hyperreal, hyper-human voice. Or, to use Cumming's terminology, they can create a virtual vocal persona which acts as an agent of musical expressivity while at the same time supplanting or reconstituting the initial subjective character of the singer's voice.

\section{Jonathan Sterne and Schizophonia}

The other consequence of Auto-Tune's tendency to supplant the physiological grain of the singer's voice with a synthetic, digitally produced vocal grain and virtual persona is the phenomenon Jonathan Sterne cites as schizophonia. Sterne traces the history of audio reproduction and sound recording technologies, from telephones to phonographs to radio to magnetic tape, exploring the boundaries between what is and is not considered sound, and the interaction between human bodies and technology. Sterne provides theoretical context for his particular notion by first raising a number of what he calls "semiexperiential definitions of modern sound-reproduction technologies"182:

\footnotetext{
182 Jonathan Stern, The Audible Past: Cultural Origins of Sound Reproduction (Durham: Duke University Press, 2003), 19.
} 
Since the power to split sources and copies is the most common definition of sound-reproduction technology, it warrants some scrutiny. Pierre Schaeffer, the composer who pioneered musique concrète, argued that sound reproduction technologies produced 'acousmatic' sounds - sounds that one hears without seeing their source ... Barry Truax and R. Murray Schafer have coined the term schizophonia to describe the 'split between an original sound and its electro-acoustic reproduction' enabled by sound reproduction technologies. The Greek prefix schizo means 'split' and also has a convenient connotation of 'psychological aberration' for these authors. Truax and Schafer also argue that reproduction removes sound from its original context. ${ }^{183}$ [all emphasis Sterne's]

It is Truax and Schafer's notion of schizophonia, rather than Sterne's subsequent definition and account of sound reproduction technology, which I shall explore in relation to Auto-Tune. As mentioned above by Sterne, the phenomenon of schizophonia arises in the listener's distinction of an original sound from its electro-acoustic reproduction; between the aural sound of the voice emanating from the singer's body and the recorded sound of that voice, recreated by sound reproduction technology (such as a phonograph turntable). How then, and in what respect, can Auto-Tune be considered schizophonic, as disassociating and/ or separating a singer's voice from his or her body?

First, the issue of context must be addressed. In its initial definition and application, schizophonia refers to the disassociation of an original sound via mechanically reproduced recording. Schizophonia in this instance refers to the technological ability and symbolic effect of musical recordings to not only capture but separate sound from its original source. With regard to Auto-Tune, the disassociation of sound from source not only occurs between the original voice

183 Sterne, The Audible Past, 20. 
and the sound reproduction technology, but also between the original voice, the sound recording technology, and the sound reproduction technology.

The ability to conduct and engage in various post production editing techniques has essentially created another level of schizophonic abstraction: instead of an original aural source being disassociated through a single medium of mechanical reproduction (e.g. the playback device), the ability to edit and effect a recording through post production software creates a secondary level of disassociation. Not only does a recording which utilizes Auto-Tune disassociate sound from an original source, but the final, post production recording is itself a disassociation from the initial recording of the original sonic source. To hear either the Auto-Tune Cher-effect or pitch correction is to hear an edited, effected copy of an initial vocal recording, which itself is a copy apart from an original, aural vocal performance. Figure 1, below, demonstrates the conventional process of schizophonia (as explored by Sterne) where no post-production processes have been applied, while Figure 2 postulates the process of schizophonia as it functions in response to the application of Auto-Tune to a recording.

- Figure 1: A Singular Occurrence of Schizophonia

Original Sound -.---.-> Recording -..--->> Playback

- Table 3: A Dual Occurrence of Schizophonia

Original Sound -----> Recording -----> Post-Production -----> Playback 
The Auto-Tuned voice, therefore, has essentially undergone a two-fold schizophonic split. The connection a vocalist has to their final, Auto-Tuned recording is a post-production copy of a recorded copy of their actual, original voice.

Informed by Baudrillard's theory above, one could postulate that the phenomenon of schizophonia could be considered a form of auditory simulacrum, one which symbolizes through distortion and disassociation a vocal hyperreality, rather than the representation of an original, aural reality. Schizophonia could also tie into Cumming's theory of a Peircean semiosis of music: because schizophonia is the separation of sound from its source, and since recorded music is fundamentally sound separated from its source and recreated through playback technology, it could be postulated that schizophonia is a process which de-emphasizes Secondness, the connection between the bodily grain as Representamen and a corresponding human Object. Because recorded music is primarily sound divorced from the body, instrument or apparatus which produces said sound, Secondness is therefore de-emphasized over Firstness and Thirdness. It is the phenomenon of schizophonia within the listener which (somewhat indirectly) contributes to Cumming's assertion that music signifies primarily through Firstness and Thirdness, de-emphasizing Secondness, since schizophonia sheds the body which produces musical sound and consequently the Object to which it could potentially refer. In any event, the task of identifying the Object and Secondness of music remains an issue for Cumming, and multiple levels of schizophonia only exacerbate and perpetuate 
this challenge, since they elaborate upon (and help to explain) the sensation of hearing musical sound as disassociated and disembodied from its original source.

\section{Conclusion}

In this chapter, Auto-Tune has been examined from a number of theoretical perspectives, which have been deployed in order to understand a variety of important aspects: the virtual persona presented by Auto-Tune, how that persona functions as an agent of musical expressivity according to a Peircean regime of signification, what unique semiotic grain it produces, how that grain produces a hyperreal vocal form, and how the application of Auto-Tune to a recording can be considered schizophonic. Having concluded such examinations, what is clear about Auto-Tune is that its application to a vocal recording has a significant impact on not only the sound, but also on the perception and interpretation of that recording. Hearing an Auto-Tuned recording of a singer's voice changes not only the manner in which one hears or perceives that voice, but also how one interprets and comprehends the symbolism and meaning of that recording. As the insights of this chapter have shown, the application of Auto-Tune to a vocal recording is neither frivolous nor superficial: applying Auto-Tune to a vocal recording entails immediate and profound influence upon both the recording, and upon the listener's experience and interpretation of that recording. 


\section{VI - Conclusion: Auto-Tune and Popular Culture}

In the preceding chapters of this thesis, Auto-Tune has been examined from a variety of theoretical and aesthetic perspectives, all of which have explored the operation and significance of Auto-Tune within a musical context and practice. However, over the past several years, during which Auto-Tune has become a clearly iconic and prominent sound within popular music, the effect has also managed to expand beyond the realm of music into a much larger popular culture. Popular musicians' use of Auto-Tune has garnered a certain musical caché which helping to spark a broader pop culture fascination with the effect. Pop cultural fascination with Auto-Tune has, over the last several years, begun to not only permeate but also to influence a number of popular culture mediums such as television and the Internet.

In this concluding chapter, I will briefly highlight and explore various ways Auto-Tune has extended from a popular music post production tool into a widely recognized pop culture trope, appearing in -- and stirring controversy on -television shows, and also being utilized on the Internet as a tool for the purposes of satire and parody. The musical and cultural success of Auto-Tune has also lead to an increase in competing pitch correction and vocal effect software and technologies, which strive to both outperform and super-ceding Auto-Tune as the preferred vocal pitch correction/effect software suite. Finally, I shall conclude this chapter and the thesis by returning full circle to the reactions of popular musicians to popular culture's recent fascination with Auto-Tune, and I 
will speculate as to whether Auto-Tune can be considered a passing popular culture and/or popular music fad, or should be seen as a technology that is destined to remain an integral part of modern music production for the foreseeable future.

\section{Television}

The first instances of Auto-Tune appearing on television occurred when the first music videos featuring Auto-Tuned music began to be released to both music-oriented television outlets and popular video-streaming websites like YouTube and Vevo. Music videos for songs like T-Pain's "I'm Sprung"184 and Kanye West's "Love Lockdown"185 not only received heavy rotation on musictelevision stations but also generated millions of views online, which helped to 1) introduce the Auto-Tune vocal effect to a mass audience, and 2) kindle a fascination with the effect amongst said audience. That fascination quickly spread as people began sharing and posting links to these videos amongst their friends and through social networks.

That fascination soon achieved mainstream recognition as Auto-Tune began to appear on more conventional, network television programs. For instance, hip hop artist T-Pain made a guest appearance on Ellen, the popular daytime talk-show hosted by Ellen Degeneres, in which he not only

\footnotetext{
184 "T-Pain - I'm Sprung," YouTube video, 3:51, uploaded by TPainVideosVEVO on November 14, 2009, accessed February 17, 2012, http:/hwww youtube.com/watch? $v=$ XXRvDpF2FDA.

185 "Kanye West - Love Lockdown," YouTube video, 4:36, uploaded by KanyeWestVEVO on December 13, 2009, accessed February 17, 2012, http://www.youtube.com/watch?

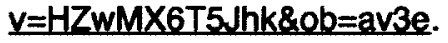


demonstrated the effect but helped to teach Ellen how to sing and rap through Auto-Tune (although Ellen innocently misinterprets the microphone rather than the software program as the source of the Cher-effect). ${ }^{186}$ Considering the size and demographic of Ellen Degeneres' audience, this appearance helped to not only introduce the Auto-Tune vocal effect to a large, predominantly caucasian audience (who may not have encountered the effect from listening to hip hop artists' music), but also to make the effect seem entertaining, familiar, and even appealing. As well, T-Pain also took the opportunity in his guest appearance on Ellen to promote his then newly released iPhone app "I Am T-Pain," ${ }^{187}$ an iPhone application in which users sing into their smartphones to produce an Auto-Tuned reproduction of their voice, a la T-Pain's unique and exaggerated settings. The app, originally produced by developer Smule with genuine Antares Audio Technologies Auto-Tune software, continues to be a consistent top-selling app in the iTunes App Store. ${ }^{188}$

Auto-Tune also made an appearance in an episode of South Park, ${ }^{189}$ in which hip hop artist Kanye West becomes publicly embroiled in the comprehension of a punchline to a joke written by one of the show's main characters. The joke, which implies that the interlocutor is a "gay fish," prompts

\footnotetext{
186 "Ellen Auto-Tuning with T-Pain!," YouTube video, 5:51, uploaded by FlackeyR on September 23, 2009, accessed February 17, 2012, http://www.youtube.com/watch?v=OvHmrShrmaM.

187 "I Am T-Pain by Smule," Smule, accessed February 28, 2011, httpilliamtpain.smule.com/.

188 "App Store - I Am T-Pain 2.0," Apple.com/ca, accessed March 28, 2012, http:// itunes.apple.com/ca/app/i-am-t-pain-2.0/id314652382?mt=8.

189 "Kanye West - Gay Fish (Episode and Song)," YouTube Video, 2:29, uploaded by chitownkid21 on April 11, 2009, accessed February 17, 2012, http://www.youtube.com/watch? $v=n m 9 N z 2 z C V 5 M$.
} 
Kanye West to publicly proclaim that he is "not a gay fish." However, in the show's conclusion, West instead accepts his aquatic homosexuality, and a musical parody of Kanye West's "Heartless" is played over the closing credits, with the chorus' original lyrics of "how could you be so heartless" altered to "because I'm a motherf*ネ*ing gay fish." The parody serves a dual purpose: lyrically it ties into the themes of the episode (and the action which occurs onscreen), but it also serves as a public lampooning of both Kanye West's persona and music, specifically his heavily Auto-Tuned 808 s and Heartbreak material. In any event, the absurdity of the episode has managed to resonate within pop culture, particularly amongst fans of South Park and/or Kanye West, even receiving lyrical reference and response from Kanye West in songs such as "Gorgeous" 190 and "Made in America."191

Other instances of Auto-Tune appearing in network television programs include an Auto-Tuned montage broadcast during the $83^{\text {rd }}$ Annual Academy Awards ceremony on February 27, 2011,192 which featured a YouTube-esque. parody video involving scenes taken from Harry Potter, Toy Story 3, The Social Network, and Twilight in which dialogue from those films was Auto-Tuned and set to a pop/dance music background track. This video exposed the Auto-Tune Cher-

\footnotetext{
190 Kanye West, "Gorgeous," My Beautiful Dark Twisted Fantasy, Roc-A-Fella Records LLC/Def Jam B003X2O6KW, compact disc, 2010.

191 Jay-Z and Kanye West, "Made in America," Watch the Throne, Roc-A-Fella Records LLC/ Universal Music B005BQLCBO, compact disc, 2011.

192 "Oscar 2011 Auto-Tune - Academy Awards 2011 Oscars," YouTube video, 1:32, uploaded by MarkinVideos on February 28, 2011, accessed February 17, 2012, http://www.youtube.com/ watch?v=YKOP5TAF_0w.
} 
effect to a mass audience (estimated in the hundreds of millions), and in addition, the fact that the ceremony's producers were not only aware of, but participated in and broadcast their own Auto-Tune spoof provides a clear indication of the extent to which Auto-Tune had become a widespread (and recognizable) cultural phenomenon.

However, not all instances of Auto-Tune in conventional network television programs have been positive: in 2010, a controversy erupted in England when it was discovered that Auto-Tune had been applied to contestants of the reality television shows Britain's Got Talent and The X-Factor. While the application of Auto-Tune to contestants of a reality television talent show itself seems like a betrayal of the basic premise of reality television, the controversy was exacerbated when it was discovered that applying Auto-Tune was a common practice in not only making contesțants sound better but also in making certain contestants sound worse. ${ }^{193}$ The application of Auto-Tune in The X-Factor was most discernible when an 18-year-old contestant named Gamu Nhengu performed a cover version of "Walking on Sunshine" by Katrina and the Waves, ${ }^{194}$ particularly in the song's second verse, where Auto-Tune's pitch correction drone can be heard in Nhengu's vocals. ${ }^{195}$ The controversy sparked disenchantment amongst the show's fans, many of whom vented their

${ }^{193}$ Chris Matyszczyk, "Will 'American Idol' be wrecked by Auto-Tune affair?," Cnet News, August 25, 2010, accessed February 17, 2012, http://news.cnet.com/8301-17852 3-20014689-71.html.

194 Katrina and the Waves, "Walking on Sunshine," Walking on Sunshine: The Greatest Hits Of, EMI Music Canada B000024R45, compact disc, 2005, originally released in 1983.

185 "X Factor admits tweaking vocals," BBC News, August 23, 2010, accessed February 17, 2012, http://www.bbc.co.uk/news/entertainment-arts-11056050. 
disapproval of the use of Auto-Tune on various social networks, while mainstream news organizations like the UK newspaper The Guardian published biting, cynical reviews of the practice:

But I still can't shake the nagging feeling of being conned. The whole point of The $X$ Factor - the reason millions tune in - is because it dramatises little moments of human triumph against the odds: the single mum who never caught a break, the pool cleaner giving his all for his kids. Adding Auto-Tune doesn't improve those tear-jerking moments, it reduces them; makes them fraudulent. The implication is that these singers are not the authors of their own destiny; they are merely following a script penned by the show's creators that is set to end in a flurry of glitter cannons and another bloodless cover version at No 1 come December. It seems as though the show's producers are sneering at their own loyal viewers. ${ }^{196}$

In this instance, the application of Auto-Tune produced a detrimental outcome for both the show's producers and for the public perception and opinion of AutoTune. Unlike the friendly, playful representation of Auto-Tune as seen on Ellen, South Park, and the 83rd Annual Oscars, the Britain's Got Talent and The $X$ Factor controversies compelled viewers to question not only their expectations of realism in reality television, but the deeper ethical issues regarding whether -and/or under what conditions -- a singer's voice should be Auto-Tuned.

\section{Internet}

While Auto-Tune has managed to garner media exposure through a number of high profile television events and appearances, perhaps more importantly Auto-Tune has over the years become a thriving cultural trope on the

\footnotetext{
196 Luke Lewis, "X-Factor's Auto-Tune row strikes the wrong note," The Guardian, August 23, 2010, accessed February 17, 2012, http://www.guardian.co.uk/tv-and-radio/tvandradioblog/2010/ aug/23/x-factor-auto-tune-row.
} 
Internet. As mentioned above, pop culture fascination in the Auto-Tune vocal effect initially spread via the sharing and posting of links to music videos featuring Auto-Tune on websites like YouTube and Vevo. Websites like YouTube helped to foster the viral spread of these videos by not only hosting them, but also by enabling the linking and sharing of these videos through social networks. It is fitting, then, that YouTube has become a domain where Auto-Tune is used not only by musicians to promote their music, but also by regular users who have established a practice of posting their own remix videos in which the Auto-Tune vocal effect is used as a tool for satire and parody. Examples of such videos include the Super-Mario remix, ${ }^{197}$ in which snippets of the iconic Nintendo video game character's dialogue is Auto-Tuned and remixed to an uptempo dance music track, as well as Auto-Tuned remixes of an Apple press conference, ${ }^{198}$ an Auto-Tuned crying baby, ${ }^{199}$ and the Auto-Tuned remix of Serene Branson, a Los Angeles entertainment television reporter who suffered a mild on-air stroke outside the 2011 Grammy Awards. 200 These videos, and many others like them, utilize Auto-Tune in order to satirize and parody both mass media and popular culture, by taking pre-existing footage, remixing the footage to produce a new,

197 "Super Mario Autotuned," YouTube video, 0:13, uploaded by barelypolitical on January 1 , 2010, accessed February 17, 2012, http:/www.youtube.com/watch?v=TrDRVa1Exkc.

198 " Auto-Tune: Phil Schiller I Mac Kicks Ass Song," YouTube video, 1:24, uploaded by iTunedSteveJobs on July 8, 2011, accessed February 17, 2012, http://mwwyoutube.com/watch? $\mathrm{V}=$ ax89xyULOR0\&feature $=$ player embedded.

199 "Baby T-Pain... (funny)," YouTube video, 0:41, uploaded by hakarolo on August 11, 2008, accessed February 17, 2012, http://wmw youtube.com/watch? $v=2$ 4AxzyhCPY.

200 "Serene Branson (Jake Benson Auto-Tune Remix)," YouTube video, 0:59, uploaded by brucenitro on February 14, 2011, accessed February 17, 2012, http://www.youtube.com/watch? $v=$ das live $\mathrm{ycg}$. 
often absurd narrative, and applying Auto-Tune to the audio track in order to make the dialogue more melodic and in keeping with the musical track. It is a practice and aesthetic based upon the Internet's voracious appetite for found comedy - a primal need to laugh at things that are different or unexpected and not necessarily planned to be funny."201

This formula has proven hugely successful for Evan, Michael and Andrew Gregory, more commonly known as The Gregory Brothers, the creators behind the Auto-Tune the News YouTube series which takes television news clips, runs the vocals through Auto-Tune, and sets it to music. The series originated from a set of parody videos in 2008 in which footage from United States Presidential election debates were songified, a term referring to the practice where

pitch correcting software [is used to] manipulate portions of recorded speech like musical notes, making them higher or lower, longer or shorter, and arranging them into something that sounds like a tune ... to these newly minted vocals, they add instrumentation - guitars, bass, keyboards, backing vocals, synthesized handclaps, [etc.].202

Since April 2009, The Gregory Brothers have produced a number of videos that have songified various television news stories and pop culture clips into hilarious, ironic parodies of both network news and mass media (as well as producing the Auto-Tune montage broadcast during the 83rd Annual Academy Awards ceremony). ${ }^{203}$ The Gregory Brothers achieved their most successful -- and viral --

201 David Itzkoff, "The Gregory Brothers Auto-Tune the Internet," New York Times Magazine, August 11, 2011, accessed February 17, 2012, http://www.nytimes.com/2011/08/14/magazine/ the-gregory-brothers-auto-tune-the-intemethtml?partner=rss\&emc=rss\&pagewanted=alli.

202 Itzkoff, "The Gregory Brothers."

203 Itzkoff, "The Gregory Brothers." 
parody with their "Bed Intruder Song,"204 in which Huntsville, Alabama resident Antoine Dodson recounts an incident in which an intruder broke into their home, attempted to sexually assault Dodson's sister, and was chased off by Dodson when he overheard the struggle. In his on-camera interview, Dodson described the incident -- in an equally enraged and lyrical cadence -- by saying:

Well, obviously, we have a rapist in Lincoln Park. He's climbing in your windows, he's snatching your people up, trying to rape 'em. So y'all need to hide your kids, hide your wife and hide your husband because they're raping everybody out here. ${ }^{205}$

That soundbite became the highly musical and lyrical hook to the "Bed Intruder Song," a video which has achieved a massive, viral exposure (having been viewed over 100 million times) as well as spawning a cottage industry of dedicated iPhone apps, an official single released on iTunes, and even a website where one can purchase a Bed Intruder Costume 206 based on Antoine Dodson's clothing from the video (which subsequently became a popular Halloween costume in 2010).

Other forums where Auto-Tune generates and receives mass recognition include Twitter, where the hashtag \#autotune is a source of criticism, commentary, humour, wit, and parody amongst the social network's users as well as an ongoing barometer of Auto-Tune's status in popular culture. In addition, the

\footnotetext{
204 "Auto-Tune the News: BED INTRUDER SONG!!! (now on iTunes)," YouTube video, 2:08, uploaded by schmoyoho on July 31, 2010, accessed February 17, 2012, http://wwwhyoutube,com/ watch? $v=h M$ MizW $279 d w$.

205 Itzkoff, "The Gregory Brothers."

206 "Bed Intruder Costume: Antoine Dodson Halloween Costume," The Mirza Agency, accessed February 17, 2012, http:/hww.bedintrudercostume.com/.
} 
popular website Know Your Meme, which chronicles and describes current online and/or pop cultural trends, posted a widely viewed article featuring Weird Al Yankovic in which they explain the origin of Auto-Tune and explore pop culture's fascination with the technology. ${ }^{207}$ The website, although humorous, provides a poignant insight into the process by which Auto-Tune managed to captivate popular culture. Know Your Meme posits that Auto-Tune has undergone a fourstage process:

4. Introduction: the first instances of Auto-Tune being applied to, and recognized within popular music, which fascinated both audiences and musicians.

5. Overexposure: the use of the Auto-Tune vocal effect became so extensive in popular music that it turned into a source for aesthetic criticism and discussion; or worse, a joke.

6. Parody and Remix: the stage at which Auto-Tune was applied to non-musical sources, generating humour through various YouTube remix and parody videos. The Auto-Tune vocal effect, in becoming a tool for parody, recontextualizes the video being parodied by shifting emphasis from "esoteric mockery into legitimate parody; while making fun of something is easy, parody requires a study of both technique and form before creating its own recontextualization. ${ }^{208}$ This also recontextualizes Auto-Tune itself, from a joke in-itself into a tool for creating a joke. And finally,

\footnotetext{
207 Chris Menning, "Auto-Tune," Know Your Meme, 2010, accessed February 17, 2012, http:ll knowyourmeme.com/memes/auto-tune.

208 Chris Menning, "Auto-Tune," Know Your Meme, 2010, accessed February 17, 2012, http:ll knowyourmeme,com/memes/auto-tune.
} 
7. Equilibrium: a state where a once overused effect or technique becomes a permanent fixture of a creative, cultural, and/or musical landscape, though in a more restrained, conventionalized manner.

While Know Your Meme tinges its sociological insights into Auto-Tune's interactions with pop culture with irreverent humour, their analysis remains valid and pertinent: while the initial experimentation with Auto-Tune in popular music may have led to a degree of overuse and/or abuse of the technology (as did, for example, stereo-panning and turntable scratching upon their initial discovery and early experimentation), eventually the meme will peak through various parodies and remixes and subsequently settle into a long term equilibrium, establishing the Auto-Tune vocal effect as a permanent, though more conservatively used, musical effect. The fundamental message underlining the Know Your Meme Auto-Tune video is that while the vocal effect may have been overused and/or abused by early adopters, its subsequent use as a vehicle for humourous parody and remixes will help to reconsider attitudes regarding the vocal effect, contributing to an eventual acceptance and conventionalization of Auto-Tune amongst the pantheon of other musical techniques and effects.

\section{Music Technology}

So how has Antares Audio Technologies, the maker of the Auto-Tune software suite, responded to this pop cultural process of introduction, overexposure, parody/remix, and equilibrium? Marco Alpert, Vice President of Marketing for Antares, in regard to the use of Auto-Tune in a television advertisement for fast-food chain Wendy's, replied: 
We're thrilled to have our brand out in the general world of pop culture. When it's made it into Wendy's commercials, we know that we've definitely moved beyond the rather rarified boundaries of the professional audio-technology world. Controversy is good for the Auto-Tune brand, and kind of fun to boot.209

Alpert's positive regard for Auto-Tune's parodic use is sustained by his broader recognition of Auto-Tune's expansion beyond the audio-technology world and its transformation into a much larger pop culture meme. As well, the expansion of Auto-Tune into wider pop cultural recognition bodes well for the long term viability and utilization of Auto-Tune within the audio-technology world. With the eventual equilibrium of the Auto-Tune vocal effect, Antares Audio Technology's Auto-Tune vocal suite is well situated to become a permanent fixture within the audio production process, alongside other essential audio recording software such as Pro-Tools.

However, Antares Audio Technology's Auto-Tune is not the only vocal effect software suite competing for a place within the arsenal of modern, digital audio recording and editing software: the Nectar Complete Vocal Suite, produced by iZotope, is a vocal software suite which not only offers pitch correction similar to Auto-Tune, but also a "manual note editor, breath control, gate, compressors, saturation, EQ, de-esser, doubler, limiter, reverb, delay and tons of built-in style presets"10 which enable the user to achieve and/or imitate particular vocal sounds and styles (such as "the 60s Motown sound, the early 90s grunge rock

209 Charlie Moran, "Thank You For Killing Auto-Tune, Wendy's!," Advertising Age Blog, June 11, 2009, accessed February 17, 2012, thittp:/ladage.com/article/songs-for-soap/killing-auto-tunewendy-s/1372321.

210 "Nectar Complete Vocal Suite by iZotope," iZotope Inc., accessed February 17, 2012, http:ll unw.izotope.com/products/audio/nectarl. 
sound, a radio-ready podcasting sound, a subtle jazz vocal or a modern pop sound -- everything from delicate improvements to highly-produced robotic pitch

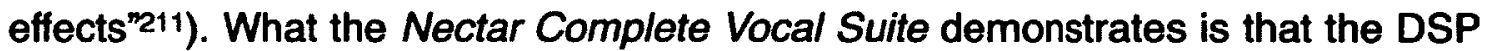
and synthesis capabilities of Auto-Tune can be expanded even further, allowing for the ability to imitate, replicate and/or simulate a particular, recognizable, even historical vocal sound. Not only can pitch correction software clandestinely alter a singer's pitch, it can also be used to simulate the aura of an iconic vocal staging.

Another competing vocal pitch correction technology comes from Digitech, an audio-technology company famous for developing iconic guitar effects (such as the Whammy pedal), who have developed an entire line up of vocal harmonizing and effects pedals. In particular, the Vocalist Live 5 Intelligent Vocal Harmony and Effects Processor 212 is a stomp-box pedal which enables a singer to capture and produce four additional vocal harmonies on top of the singer's voice, in both a studio recording and a live performance. The Live 5 includes built-in pitch correction, compression, a noise gate, two-band EQ, reverb, delay, the ability to adjust between tight and loose harmonies, as well as distinguish between male and female backup vocal timbres. The Live 5 achieves pitchperfect vocal harmonies by analyzing the singer's lead vocals, as well as the chords and notes played by any accompanying guitar(s) or piano(s), and automatically pitch corrects based on those harmonies, rather than having the

\footnotetext{
211 "Nectar Complete Vocal Suite by iZotope," iZotope Inc., accessed February 17, 2012, http:ll wnwizotope.com/products/audio/nectarl.
}

212 "Digitech Vocalist Live 5: Intelligent Vocal Harmony and Effects Processor," Harman Professional, accessed February 17, 2012, http://vocalistpro.com/en-US/products/ive-5. 
song's key and/or chord progression inputed into the device (as is often the case in Auto-Tune). The distinct advantage of the Live 5 is that, by being a physical stomp-box, a singer can not only pitch correct but also harmonize his or her voice in a live performance more effectively than Auto-Tune (which can be applied in a live performance, but requires an external laptop and is not as efficient at instantaneous, clandestine pitch corrections).

What these competing vocal effect technologies demonstrate is that the field of vocal effect technology is not only expanding but is innovating in both rapid and creative ways, and presenting new possibilities in which vocalists can effect, harmonize, and/or pitch correct their vocals in a variety of musical situations and environments. While Auto-Tune may have been the first, and subsequently most recognizable pitch correction and vocal effect brand, it is not the only one. Its competitors are not only increasing in number, but innovating in a variety of ways which will have a significant impact on both a singer's performance and how his or her vocals will be recorded and edited in the near future.

\section{Popular Music}

In light of pop culture's recent fascination with the Auto-Tune vocal effect, as both an overused and/or abused joke and a vehicle for online parody and satire, how have musicians responded and reacted to the use of Auto-Tune beyond the realm of the recording studio, and what does pop culture's fascination with Auto-Tune mean for the future of this vocal technology? 
Some musicians have made public declarations and demonstrations against the perceived abuse of Auto-Tune within their industry. For example, at the 2009 Grammy Awards red-carpet ceremony indie-rock group Death Cab For Cutie called for a public ban on Auto-Tune, pinning baby blue ribbons to the lapels of their clothes in a protest against the overuse of Auto-Tune. As an indierock band, Death Cab For Cutie adhere to the indie-rock aesthetic of realism and authenticity, and criticize the (over)use and reliance upon music technology to modify, enhance, or alter the realism of a musical recording. As journalist Sean Michaels noted in the UK newspaper The Guardian, "the American band are proving their indie-rock credentials and fighting the 'authentic' fight against $T$ Pain, Kanye, and a thousand digitally altered voices." ${ }^{213}$ Frontman Ben Gibbard (and other members of the band) further elaborate upon their views by saying:

"We want ... to bring back the blue note," Gibbard explained to MusicRadar. "The note that's not so perfectly in pitch and gives the recording soul and real character. It's how people really sing." Gibbard bemoaned the loss of "actual people singing and sounding like human beings," condemning the advent of warbly, cyborg tones. "A little use is OK, but there is a difference between 'use' and 'abuse," he said. "Otherwise," added bassist Nick Harmer, "musicians of tomorrow will never practice. They will never try to be good, because yeah, you can do it just on the computer."214

Indie-rock bands like Death Cab For Cutie value so highly the ability to play their instruments and sing their vocals that authenticity in musical performance comprises a core, fundamental criteria of the Indie-rock subgenre. To these

\footnotetext{
213 Sean Michaels, "Death Cab for Cutie declare war on Auto-Tune abuse," The Guardian, February 11, 2009, accessed February 17, 2012, http://www.guardian.co.uk/music/2009/feb/11/ death-cab-for-cutie-aute-tune.
}

214 Michaels, "Death Cab for Cutie." 
musicians, the prospect of using computer software programs such as Pro-Tools and Auto-Tune to overcome their musical or performative shortcomings is aesthetically at odds with their (sub)genre's notions and conventions of realism in musical performance and recording. As explored previously in Chapter IV, the core aesthetic values and conventions of a musician's affiliated genre can greatly influence a musician's opinions regarding both the application of music technology and how their music is either performed and/or recorded.

For other musicians, however, their denunciation of Auto-Tune has been partly influenced by pop culture's contribution to the overexposure and subsequent parodying of Auto-Tune. In regards to his denunciation of Auto-Tune in "D.O.A (Death of Auto-Tune)," Jay-Z commented that

"In hip hop, our job is once a trend becomes a gimmick, to get rid of it. We've done that since the beginning of time," explained Jay$Z$ of his aversion to Auto-Tune. "Now people are using Auto-Tune even in [fast food chain] Wendy's commercials, and it's like, 'Oh no! That has to go!' Its become part of main culture. It's the same thing like when the old lady in Oregon starts saying, 'Bling, bling.' It's like, I'm never saying that again'.215

The overexposure and subsequent parodying of Auto-Tune in popular culture affected the vocal effect's cultural caché to such a degree that using the effect within one's music had -- at least by late 2009 and early 2010 -- begun to seem passé. Since the release of Jay-Z's 2009 album The Blueprint 3, other artists (including Kanye West) have begun to curtail their use of Auto-Tune; West's 2010 album My Beautiful Dark Twisted Fantasy represents a more restrained

215 "Jay-Z: 'The Anti-Auto-Tune Record Was Kanye's Idea," NME.com, July 20, 2009, accessed February 17, 2012, http://www.nme.com/news/jay-z/46206. 
counterpoint to his all Auto-Tuned 2008 album $808 \mathrm{~s}$ and Heartbreak, for example. The most telling point of all regarding Auto-Tune's waning popularity musicians may be the news that T-Pain, the innovator of the Auto-Tuned hip hop idiom, declared in June of 2011 that he was moving on from Auto-Tune: "I'm done with Auto-Tune ... I vow right here, right now, to never use Auto-Tune again."216 Such a reversal is potentially of great significance, for both the artist and for the technology. However, T-Pain also stipulated in his declaration that he was working with iZotope -- makers of the competing Nectar Complete Vocal Suite -to create his own, unique vocal processing software program. The result of this collaboration is The T-Pain Effect Suite, ${ }^{217}$ which features a T-Pain Effect plugin (which allows DAW software such as Garageband, Logic, Pro-Tools, etc., to apply his unique Auto-Tuned vocal effect to a recording), a Pro-Tools-esque Engine (which can be used to arrange recorded vocal and instrumental recordings on a computer, as well as publish those recordings online to music social network SoundCloud), and a virtual drum machine (which both provides preset drum samples and allows producers to create their own rhythms and beats). While T-Pain may have declared his artistic intention to move on from his signature Auto-Tune vocal effect, the artist still exercises a certain degree of economic interest in developing and marketing his unique brand of vocal effects processors and software.

\footnotetext{
${ }^{216}$ Gil Kaufman, "T-Pain Vows to Put Auto-Tune On Permanent Lock-Down," MTV News, June 2, 2011, accessed February 17, 2012, http://rapfix.mtv.com/2011/06/02/t-pain-vows-to-put-autotone-on-permanent-lock-down/\#more-15805.

217 "The T-Pain Effect: Make Beats. Record Vocals. Share I IZotope," IZotope Inc., accessed February 17, 2012, http:/www.izotope.com/products/audio/tpaineffect.
} 
Nevertheless, as Jay-Z, T-Pain, and Kanye West make evident, artists are recognizing that the Auto-Tune vocal effect has become overexposed, and as the hundreds (even thousands) of Internet Auto-Tune remix and parody videos demonstrate, the vocal effect has become a tool both of and for satire and parody. Consequently, since 2010 the use of the Auto-Tune vocal effect in popular music has begun to decline, occurring less frequently and in more restrained and conservative ways. For instance, the use of Auto-Tune on Jay- $Z$ and Kanye West's 2011 album Watch the Throne is far more restrained than in previous hip hop albums, having primarily been limited to the bridge in album opener "No Church in the Wild" and the Nina Simone sample which comprises the chorus of "New Day." The more restrained use of Auto-Tune demonstrated on Watch The Throne, as well as other recent hip hop albums from Kanye West, TPain, Drake, Lil Wayne and other hip hop artists, likely indicates that the AutoTune vocal effect is beginning to enter a state of equilibrium. This equilibrium, like the practices and techniques of stereo-panning and turntable scratching before Auto-Tune, will help to establish Auto-Tune -- both its pitch correction and vocal effect capabilities -- as conventionalized, accepted musical production techniques. 


\section{Bibliography}

Aaron, Charles. "Album Review: Kanye West's '808s and Heartbreak." Spin Magazine, November 25, 2008. Accessed February 16, 2012. http://www.spin.com/blog/album-review-kanyewests-808s-and-heartbreak.

Barthes, Roland. "The Grain of the Voice." Image-Music-Text. Translated by Stephen Heath. New York: Hill and Wang, 1977.

Baudrillard, Jean. Simulacra and Simulation. Translated by Sheila Faria Glaser. University of Michigan Press, 1994.

Cobley Paul and Jansz, Litza. Introducing Semiotics. Edited by Richard Appignanesi. Thriplow, Royston, UK: Totem Books, 1999.

Cumming, Naomi. The Sonic Self: Musical Subjectivity and Signification. Bloomington: Indiana University Press, 2000.

Davies, Stephen. Musical Meaning and Expression. Ithaca: Comell University Press, 1994.

DeCurtis, Anthony. "Word." The Vibe History of Hip Hop. Edited by Alan Light. London: Plexus Publishing, 1999.

Diehl, Matt. "Pop Rap." The Vibe History of Hip Hop. Edited by Alan Light. London: Plexus Publishing, 1999.

Dombal, Ryan. "Kanye West: My Beautiful Dark Twisted Fantasy Album Review." Pitchfork Media, November 22, 2010. Accessed February 16, 2012. http://pitchfork,com/reviews/albums/14880my-beautiful-dark-twisted-fantasyl.

Frith, Simon. Performing Rites. Cambridge: Harvard University Press, 1996.

Gissen, Jesse. "Recovery or My Beautiful Dark Twisted Fantasy?: Which Is the Best Album of 2010?." XXLMagazine, December 7, 2010. Accessed February 16, 2012. http:ll

www.xx/mag.com/blogoers/2010/12/recovery-or-my-beautiful-dark-twisted-fantasyl.

Huang, Edwyn. "Hate It Or Love It: A Hip Hop Analysis Of Drake." HipHopDX, August 29, 2009. Accessed February 16, 2012. hitt://wmw.hiphopdx.com/index/editorials/id.1398/title.hate-it-orlove-it-a-hip hop-analysis-of-drake.

Itzkoff, David. "The Gregory Brothers Auto-Tune the Internet." New York Times Magazine, August 11, 2011. Accessed February 17, 2012. http://wmw.nytimes.com/2011/08/14/magazine/thegregony-brothers-auto-tune-the-internet.html?partner=rss\&emc=rss\&pagewanted=all\#.

Jarenwattananon, Patrick. "Kanye West, Auto-Tune Crooner." NPR, November 30, 2008. Accessed February 16, 2012. http://wmw.npr.org/blogs/sundaysoapbox/2008/11/ kanye west autotune crooner $1 \mathrm{html}$.

"Jay-Z: 'The Anti-Auto-Tune Record Was Kanye's Idea." NME.com, July 20, 2009. Accessed February 17, 2012. http:/hww.nme.com/news/jay-z/46206. 
Kaufman, Gil. "T-Pain Vows to Put Auto-Tune On Permanent Lock-Down." MTV News, June 2, 2011. Accessed February 17, 2012. http://raptix.mtv.com/2011/06/02/-pain-vows-to-put-autotone-on-permanent-lock-down/\#more-15805.

Lacasse, Serge. "Listen to My Voice': The Evocative Power of Vocal Staging in Recorded Rock Music and Other Forms of Vocal Expression." PhD diss., University of Liverpool, 2000.

Lewis, Luke. "X-Factor's Auto-Tune row strikes the wrong note." The Guardian, August 23, 2010. Accessed February 17, 2012. http://wmw.quardian.co.uk/tv-and-radio/tvandradioblog/2010/aug/ 23/x-factor-auto-tune-row.

Matyszczyk, Chris. "Will 'American Idol' be wrecked by Auto-Tune affair?." Cnet News, August 25, 2010. Accessed February 17, 2012. http://news.cnet.com/8301-17852 3-20014689-71.html.

Michaels, Sean. "Death Cab for Cutie declare war on Auto-Tune abuse." The Guardian, February 11, 2009. Accessed February 17, 2012. http://www, guardian.co.uk/music/2009/feb/11/death-cabfer-cutie-auto-tune.

Milner, Greg. Perfecting Sound Forever: An Aural History of Recorded Music. New York: Faber and Faber Inc., 2009.

Moran, Charlie. "Thank You For Killing Auto-Tune, Wendy's!." Advertising Age Blog, June 11, 2009. Accessed February 17, 2012. <http://adage.com/article/songs-for-soap/killing-auto-tunewendy-s/1372321.

MTV News Staft. "Black Eyed Peas, Lady Gaga Top iTunes All-Time Most-Downloaded Songs." MTV.com, February 11, 2010. Accessed February 16, 2012. http://www.mtv.com/news/articles/ 1631774/bep-gaga-top-itunes-allime-mostdownloaded-songs.jhtml.

NWSO. "BLOG: I Heart Kanye West (A Preview of Sorts)." XXLmag.com, October 28, 2008. Accessed February 16, 2012. http:/www.xx/mag.com/bloggers/2008/10/i-heart-kanye-west-apreview-of-sorts/comment-page-2l.

NWSO. "What's Kanye West's Third Best Album? - "808s" Don't Count." XXL Magazine, July 29, 2010. Accessed February 16, 2012. http://www.xxlmag.com/bloggers/2010/07/what's-kanye-

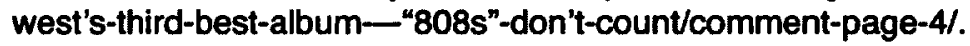

Paine, Jake. "Kanye West's My Beautiful Dark Twisted Fantasy." HipHopDX, November 15, 2010. Accessed February 16, 2012. http://www. hiphopdx.com/feature/30in30/page8,php.

Pinch, Trevor and Trocco, Frank. Analog Days: The Invention and Impact of the Moog Synthesizer. Harvard University Press, 2004.

Reid, Shaheem. "Mary J. Blige Defends Auto-Tune: 'To Each His Own." MTV.com, June 11, 2009. Accessed February 16, 2012. http://unw.mtv.com/news/articles/1613803/mary-j-blige-defendsautotune.jhtml.

Richards, Chris. "808s and Heartbreak by Kanye West." The Washington Post, November 24, 2008. Accessed February 16, 2012. http://www.washingtonpost.com/wp-dyn/content/article/ 2008/11/23/AR2008112302506.html.

Rogerson, Ben. "Kanye West says Auto-Tune makes him a better singer." MusicRadar.com, December 2, 2008. Accessed February 16, 2012. http:/www.musicradar.com/news/tech/kanyewest-says-auto-tune-makes-him-a-better-singer-185278. 
Rosen, Jody. "Kanye West: 808s and Heartbreak Review." Rolling Stone, December 11, 2008. Accessed February 16, 2012. http://hww.rollingstone.com/music/albumreviews/808sheartbreak-20081211.

Schloss, Joseph Glenn. Making Beats: The Art of Sample-Based Hip-Hop. Middletown, CT: Wesleyan University Press, 2004.

Shuker, Roy. "Genre; Meta Genres; Subgenres." Key Concepts in Popular Music. London/New York: Routledge, 1998.

Shuker, Roy. "Pop Music." Key Concepts in Popular Music. London/New York: Routledge, 1998.

Shuker, Roy. "Rap; Hip Hop." Key Concepts in Popular Music. London/New York: Routledge, 1998.

Sillitoe, Sue. "Recording Cher's Believe." Sound on Sound, February 2009. Accessed February 16, 2012. http:/www. soundonsound.com/sos/feb99/articles/tracks661.htm.

"Singing: 20"th Century." In The New Grove Dictionary of Music and Musicians, Second Edition, Volume 23. Edited by Stanley Sadie, Executive Editor John Tyrrell. London: Macmillan Publishers Limited, 2001.

Stern, Jonathan. The Audible Past: Cultural Origins of Sound Reproduction. Durham: Duke University Press, 2003.

Szwed, John F. "The Real Old School." The Vibe History of Hip Hop. Edited by Alan Light. London: Plexus Publishing, 1999.

Tracy, Christopher John. "Pitch-adjusting software brings studio tricks." Boston Herald, February 19, 2007. Accessed February 16, 2012. http://pqasb.pqarchiver.com/bostonherald/access/ 1218937871 $\mathrm{htm}$ ? $\mathrm{dids}=1218937871: 1218937871$ \&FMT =ABS\&FMTS $=A B S: F T \& d a t e=F e b$ $\pm 19 \% 2 \mathrm{C}+2007$ \&author $=\mathrm{CHRISTOPHER}+\mathrm{JOHN}+\mathrm{TREACY} \& p u b=B$ Bston tHerald\&edition $=\&$ startpage $=32 \&$ de $c=M U S \mid C+\% 3 B+P i t c h$-adjusting + software $+b r i n g s+$ studio tricks.

Trust, Gary. "Ask Billboard: Twice Is Nice, Twice, for Adele." Billboard.com, November 5, 2011. Accessed March 28, 2012. http://www.billboard.com/\#/column/chartbeat/ask-billboard-twice-isnice-twice-for-adele-1005477572.story?page $=2$.

Walker, Margaret. "Naomi Cumming. The Sonic Self: Musical Subjectivity and Signification." Discourses in Music: Volume 4 Number 1 (Fall 2002). Accessed February 17, 2012. ISBN 0-253-33754-2. http://umw. discourses.ca/v4n125.html.

Warner, Timothy. Pop Music: Technology and Creativity: Trevor Horn and the Digital Revolution. Burlington, VT: Ashgate Publishing Ltd., 2003.

"X Factor admits tweaking vocals." BBC News, August 23, 2010. Accessed February 17, 2012. http:/hww.bbc,co.uk/news/entertainment-arts-11056050. 


\section{Websites}

Antares Audio Technologies. "Antares Auto-Tune 7 Pitch and Time Correcting Plug-in." Accessed February 16, 2012. http://www.antarestech.com/products/auto-tune-7.shtml.

Antares Audio Technologies. "AVOX Evo Antares Vocal Toolkit." Accessed January 18, 2012. http://www.antarestech.com/products/avox-evo.shtml\#throat.

Apple Inc.. "App Store - I Am T-Pain 2.0." Accessed March 28, 2012. http://itunes.apple.com/ca/ app/i-am-t-pain-2.0/id314652382? $\mathrm{mt}=8$.

Audio Masterclass. "The Audio Masterclass Music Production and Sound Engineering Online Course." Accessed February 28, 2011. http://www.audiomasterclass.com.

Billboard.com. "The Black Eyed Peas Album \& Song Chart History." Accessed March 28, 2012. http://www.billboard.com/artist/daft-punk/chart-histor/141900\#/artist/the-black-eved-peas/charthistory/272581.

Billboard.com. "Drake Album \& Song Chart History." Accessed March 28, 2012. http:/l www.billboard.com/artist/daft-punk/chart-history/141900\#/artist/drake/chart-history/855020.

Billboard.com. "Kanye West Album \& Song Chart History." Accessed March 28, 2012. http:ll www.billboard.com/artist/daft-punk/chart-history/141900\#/artist/kanye-west/chart-history/322005.

Billboard.com. "One More Time - Daft Punk I Billboard.com." Accessed March 28, 2012. http:/I www.billboard.com/artist/daft-punk/chart-history/141900\#/song/daft-punk/one-more-time/ 3072399.

Billboard.com. "T-Pain Album \& Song Chart History." Accessed March 28, 2012. http:/l www.billboard.com/artist/t-pain/690156\#/artist/t-pain/chart-history/690156.

Carlos, Wendy. "Postscript Notes On Vocoders." WendyCarlos.com. Accessed February 16, 2012. http:/hwww. wendycarlos.com/vocoders.html.

Harman Professional. "Digitech Vocalist Live 5: Intelligent Vocal Harmony and Effects Processor." Accessed February 17, 2012. http://vocalistpro.com/en-US/products/ive-5.

iZotope Inc.. "Nectar Complete Vocal Suite by iZotope." Accessed February 17, 2012. http:ll www.izotope.com/products/audio/nectarl.

iZotope Inc.. "The T-Pain Effect: Make Beats. Record Vocals. Share I iZotope." Accessed February 17, 2012. http://wwwizotope.com/products/audio/tpaineffect/.

Mellor, David. "It's true! Vinyl IS better than digital!!." RecordProducer.com, February 18, 2010. Accessed February 16, 2012, http:/hww.recordproducer.com/index.php?a=92.

Mellor, David. "When should you use Auto-Tune, and when should you avoid it like the plague?." RecordProducer.com, April 4, 2009. Accessed February 16, 2012. hitto:ll www.recordproducer.com/?a=10.

Menning, Chris. "Auto-Tune." Know Your Meme. Last modified 2010. Accessed February 17, 2012. http:/knowyourmeme,com/memes/auto-tune. 
Merriam-Webster Dictionary. "Instrumentalize." Accessed March 27, 2012. http://www.merriamwebster.com/dictionary/instrumentalize.

The Mirza Agency. "Bed Intruder Costume: Antoine Dodson Halloween Costume." Accessed February 17, 2012. http://www.bedintrudercostume.com/.

Petit-Frere, Bradley. "Against Kanye West's Use of the Auto Tune." PetitionOnline. Accessed February 16, 2012. http://www.petitiononline.com/mmest/petition.html.

RollingStone.com. "30 Best Albums of 2010." Accessed February 16, 2012. http:/l ww.rollingstone.com/music/ists/30-best-albums-of-2010-20101213/kanye-west-my-beautifuldark-twisted-fantasy-19691231.

Smule. "I Am T-Pain by Smule." Accessed February 28, 2011. http://iamtpain.smule.com/.

WikiAudio.org. "Cher-Effect." Accessed February 16, 2012. http:/len. wikiaudio.org/Cher effect.

\title{
Online Multimedia
}

\begin{abstract}
"t Auto-Tune: Phil Schiller I Mac Kicks Ass Song." YouTube video, 1:24. Uploaded by iTunedSteveJobs, July 8, 2011. Accessed February 17, 2012. http://www.youtube.com/watch? $V=a \times 89 \times y U L C R 0 \& f e a t u r e=p l a y e r$ embedded.
\end{abstract}

"Auto-Tune the News: BED INTRUDER SONG!!! (now on iTunes)." YouTube video, 2:08. Uploaded by schmoyoho, July 31, 2010. Accessed February 17, 2012. http://www.youtube.com/ watch? $v=h M t Z f W 2 z 9 d w$.

"Baby T-Pain... (funny)." YouTube video, 0:41. Uploaded by hakarolo, August 11, 2008. Accessed February 17, 2012. http://www.youtube.com/watch? $y=z$ 4AxzvhCPY.

"Ellen Auto-Tuning with T-Pain!." YouTube video, 5:51. Uploaded by FlackeyR, September 23, 2009. Accessed February 17, 2012. http://www youtube. com/watch?v=OvHmrShrmaM.

"Kaiser Chiefs - Getting Better (Beatles Cover)." YouTube video, 2:49, From a performance televised on BBC Radio 2 on June 2, 2007. Uploaded by tommarques, June 3, 2007. Accessed February 16, 2012. http://www.youtube.com/watch?v=nCcU2xtvz00.

"Kanye West - Gay Fish (Episode and Song)." YouTube Video, 2:29. Uploaded by chitownkid21, April 11, 2009. Accessed February 17, 2012. http://muw.youtube.com/watch? $v=n m 9 N z 2 z C V 5 M$.

"Kanye West - Love Lockdown." YouTube video, 4:36. Uploaded by KanyeWestVEVO, December 13, 2009. Accessed February 17, 2012. http://wuww.youtube.com/watch? $\mathrm{v}=\mathrm{HZ} \mathrm{WM} \times \mathbf{6} 6 \mathrm{~T} 5 \mathrm{Jhk} \& \mathrm{Ob}=\mathrm{av3e}$.

"Katy Perry - California Gurls (Live On Letterman)." YouTube video, 3:50. From a performance on The Late Show with David Letterman. Uploaded by KatyPerryVEVO, October 18, 2010. Accessed February 16, 2012. http://nww youtube.com/watch? $V=j u s E L 6$ drchE\&feature $=m f u$ in order\&list $=U L$.

"KRS-ONE \& BUCKSHOT "ROBOT" MUSIC VIDEO." YouTube video, 4:00. Uploaded by duckdown, July 8, 2009. Accessed February 16, 2012. http://www.youtube.com/watch? $v=i 8 R a g D s 03 c 48$ feature =player embedded. 
"Lady Gaga Interview, Ellen DeGeneres Show 11/27/2009." YouTube Video, 7:39. Uploaded by Igvideos176, April 7, 2011. Accessed February 16, 2012. http:/youtu.be/a180ZiaA4Bg.

"Oscar 2011 Auto-Tune - Academy Awards 2011 Oscars." YouTube video, 1:32. Uploaded by MarkinVideos, February 28, 2011. Accessed February 17, 2012. http://www.youtube.com/watch? $y=$ YkOP5TAF OW.

"Rebecca Black - Friday - Otficial Music Video." YouTube video, 3:48. Uploaded by rebecca, September 16, 2011. Accessed February 16, 2012. http://mww.youtube.com/watch? $v=k f V s f O S b J Y 0 \&$ list=FLa6BeLNHFOzz13sMOOPNu-g\&index $=6$.

"Serene Branson (Jake Benson Auto-Tune Remix)." YouTube video, 0:59. Uploaded by brucenitro, February 14, 2011. Accessed February 17, 2012. http://www.youtube.com/watch? $\underline{v}=$ das live $\mathrm{ycg}$.

"Super Mario Autotuned." YouTube video, 0:13. Uploaded by barelypolitical, January 1, 2010. Accessed February 17, 2012. http://www,youtube,com/watch? $v=T r D R V a 1 E x k c$.

"T-Pain - I'm Sprung." YouTube video, 3:51. Uploaded by TPainVideosVEVO, November 14, 2009. Accessed February 17, 2012. http://www youtube.com/watch?y=xRvDpF2FDA.

\section{Discography}

Aerosmith. "Sweet Emotion." Toys in the Attic. Sony BMG Music Entertainment B0000029AP, compact disc, 1994. Originally released in 1975.

The Beastie Boys. "(You Gotta) Fight For Your Right (To Party)." Licensed to III. Def Jam Recordings B0000024JN, compact disc, 1995. Originally released in 1986.

Bedingfield, Natasha. "Love Like This (feat. Sean Kingston)." Pocketful of Sunshine. Sony Music Canada Inc. B000Y14U4M, compact disc, 2008.

Beyoncé. "Crazy in Love (feat. Jay-Z)." Dangerously in Love. Sony Music Canada B000099T2L, compact disc, 2003.

Bieber, Justin. "Baby (feat. Ludacris)." My World 2.0. The Island, Def Jam Music Group B003AIBGLG, compact disc, 2010.

Black Eyed Peas. "Boom Boom Pow." The E.N.D.. Interscope B00192IV0O, compact disc, 2009.

Black Eyed Peas. "I Gotta Feeling." The E.N.D.. Interscope B00192IV00, compact disc, 2009.

Black Eyed Peas. "The Time (Dirty Bit)." The Beginning. Interscope B00475AQIU, compact disc, 2010.

Blige, Mary J. "The One (feat. Drake)." Stronger With Each Tear. Geffen Records B002UZ5G7U, compact disc, 2009.

Bon Iver. "Woods." Blood Bank (EP). jagjagwar B001MJ3MQW, compact disc, 2009. 
The Buggles. "Video Killed the Radio Star." The Age of Plastic. Island/Universal B000025709, compact disc, 2008. Originally released in 1979.

Cher. "Believe." Believe. Warner Brothers B00002430K, compact disc, 1998.

Daft Punk. "Around The World." Homework. EMI Music Canada B000000WCV, compact disc, 1997.

Daft Punk. "One More Time." Discovery. EMI Music Canada B000059MEK, compact disc, 2001.

D.J. Jazzy Jeff and the Fresh Prince. "Parents Just Don't Understand." He's the DJ, I'm the Rapper. Jive B0015XWUIQ, compact disc, 2008. Originally released in 1988.

Drake. "Best I Ever Had." So Far Gone. Young Money Records Inc./Cash Money Records Inc. B002LVAZO2, compact disc, 2009.

Eiffel 65. "Blue (Da Ba Dee)." Europop. Bliss Co. B00003GPOT, compact disc, 1999.

The Fugees. The Score. Sony Music Canada B000002B5L, compact disc, 1996.

Gnarls Barkley. "Crazy." St. Elsewhere. Atlantic Records B000F3AAUW, compact disc, 2006.

Grandmaster Flash and the Furious Five. "The Message." The Message. Sugar Hill Records B00006F2XV, compact disc, 2002. Originally released in 1982.

Hill, Lauryn. "Doo Wop (That Thing)." The Miseducation of Lauryn Hill. Sony Music Canada B00000ADG2, compact disc, 1998.

Jackson, Janet. "Got 'Til It's Gone." The Velvet Rope. EMI Music Canada B000000WEX, compact disc, 1997.

Jay-Z. The Blueprint. Universal Music Group B00005O54T, compact disc, 2001.

Jay-Z. "D.O.A. (Death of Auto-Tune)." The Blueprint III. Roc-a-Fella Records LLCMarner Music Group B002DMJM66, compact disc, 2009.

Jay-Z and Kanye West. Watch the Throne. Roc-A-Fella Records LLC/Universal Music B005BQLCBO, compact disc, 2011.

Jay-Z and Kanye West. "Made in America." Watch the Throne. Roc-A-Fella Records LLC/ Universal Music B005BQLCBO, compact disc, 2011.

Katrina and the Waves. "Walking on Sunshine." Walking on Sunshine: The Greatest Hits Of. EMI Music Canada B000024R45, compact disc, 2005. Originally released in 1983.

Ke\$ha. "Tik Tok." Animal. Sony Music Canada Inc. B002XNEll2, compact disc, 2009.

Ke\$ha. "We R Who We R." Cannibal. RCA Records/Sony Music Entertainment B0046M14RG, compact disc, 2010.

Kris Kross. "Jump." Totally Krossed Out. Sony BMG B0012GMYMI, compact disc, 2008.

Originally released in 1992.

Lady Gaga. “Monster.” The Fame Monster. Interscope B002W6Z0UK, compact disc, 2009. 
Lavigne, Avril. "Complicated." Let Go. Sony Music Canada Inc. B000066NW0, compact disc, 2002.

Lil Jon \& The East Side Boyz. "Get Low." Kings of Crunk. Universal Music Group B00006GA4S, compact disc, 2002.

Lil Wayne. "Lollipop." Tha Carter III. Young Money Records Inc./Cash Money Records Inc. B001E4IY3Q, compact disc, 2008.

Lopez, Jennifer. "Waiting For Tonight." On the 6. Sony Music Canada Inc. B00000J7RZ, compact disc, 1999.

Madonna. "Impressive Instant." Music. Warner Bros. Music B00004X01U, compact disc, 2000.

Marky Mark and the Funky Bunch. "Good Vibrations." Music For the People. Interscope Records B000026GR9, compact disc, 1991.

Maroon 5. "She Will Be Loved." Songs About Jane. Universal Music Group B00006879E, compact disc, 2002.

MC Hammer. "U Can't Touch This." Please Hammer Don't Hurt 'Em. EMI Music Canada B000002UVD, compact disc, 1990.

Minaj, Nicki. Pink Friday. Young Money Entertainment/Cash Money Records Inc. B0049D1TCM, compact disc, 2010.

Mitchell, Joni. "Big Yellow Taxi." Ladies of the Canyon. Warner Brothers Music/Reprise $B 000002 K O Q$, compact disc, 1990. Originally released in 1970.

Perry, Katy. "California Gurls (feat. Snoop Dogg)." Teenage Dream. Capitol Records LLC B003L7TTZI, compact disc, 2010.

Perry, Katy. "E.T. (feat. Kanye West)." Teenage Dream: The Complete Collection. EMI Music Canada B006XBSAP2, compact disc, 2012. Originally released in 2011.

Perry, Katy. "Last Friday Night (T.G.I.F.)." Teenage Dream. Capitol Records LLC B003L77TZI, compact disc, 2010.

Pink Floyd. "Sheep." Animals. EMI Music Canada B004ZN9UZO, 2011, compact disc, 2011. Originally released in 1977.

The Prodigy. "Breathe." Fat of the Land. XL Recordings B000002NFM, compact disc, 1997.

Public Enemy. "Fight The Power." Fear of a Black Planet. Def Jam Recordings B0000024IE, compact disc, 1990.

Reid, Alyssa. "Alone Again (feat. P. Reign)." The Game. Warner Music Group B004ZKCGNA, compact disc, 2011.

Rihanna. "Disturbia." Good Girl Gone Bad. The Island/Def Jam Music Group B0018S6YO4, compact disc, 2008. 
Rihanna. "Umbrella (feat. Jay-Z)." Good Girl Gone Bad. The Island/Def Jam Music Group B0018S6YO4, compact disc, 2008.

Salt-N-Pepa. "Push it." Hot, Cold \& Vicious. London Records B000001FHE, compact disc, 2008. Originally released in 1986.

Sir Mix-A-Lot. "Baby Got Back." Playlist: The Very Best of Sir Mix-A-Lot. Rhyme Cartel/American/ Legacy B002984ARO, compact disc, 2009. Originally released in 1992.

Smith, Bessie. "Nobody Knows You When You're Down and Out." The Essential Bessie Smith. Sony Imports B000002ADO, compact disc, 2006. Originally released in 1929.

Spears, Britney. "Toxic." In the Zone. Sony Music Canada B0000DD7LB, compact disc, 2003.

Steam. "Na Na Hey Hey Kiss Him Goodbye." Steam. Fab Distribution B0000DIJLW, 2003, compact disc. Originally released in 1969.

Sugarhill Gang. "Rapper's Delight." Rapper's Delight: The Best of the Sugarhill Gang. Warner Music Group B0000033K4, compact disc, 1996. Originally released in 1980.

Timbaland. "Give It To Me (feat. Nelly Furtado \& Justin Timberlake)." Shock Value. Universal Music Group B000NA26ZE, compact disc, 2007.

Timbaland. "If We Ever Meet Again (feat. Katy Perry)." Shock Value II. Blackground Records/ Interscope B002SGQGYI, compact disc, 2009.

Timbaland. "Say Something (feat. Drake)." Shock Value II. Blackground Records/Interscope B002SGQGYI, compact disc, 2009.

Timbaland. "The Way I Are (feat. Keri Hilson \& D.O.E.)." Shock Value. Universal Music Group B000NA26ZE, compact disc, 2007.

Timberlake, Justin. "Chop Me Up." Futuresex/Lovesounds. Sony Music Canada B000H305U0, compact disc, 2006.

Timberlake, Justin. "SexyBack." Futuresex/Lovesounds. Sony Music Canada B000H305U0, compact disc, 2006.

Timberlake, Justin. "What Goes Around (Comes Around)." Futuresex/Lovesounds. Sony Music Canada B000H305U0, compact disc, 2006.

T-Pain. Epiphany. Sony Music Canada B000POJQ9Q, compact disc, 2007.

T-Pain. Rappa Ternt Sanga. Sony Music Canada B000BF0DJC, compact disc, 2006.

U2. "Discoteque." Pop. Universal Music Group B000001EAQ, compact disc, 1997.

Usher. "Yeah!." Confessions. BMG/Zomba Recording LLC B0002ZYDKQ, compact disc, 2004.

Vanilla Ice. "Ice Ice Baby." To The Extreme. EMI Music Canada B00000DRBV, compact disc, 1999. Originally released in 1990.

West, Kanye. 808s and Heartbreak. Roc-A-Fella Records LLC B001FBIPFA, compact disc, 2008. 
West, Kanye. My Beautitul Dark Twisted Fantasy. Roc-A-Fella Records LLC/Def Jam B003X2O6KW, compact disc, 2010.

West, Kanye. "Gorgeous." My Beautiful Dark Twisted Fantasy. Roc-A-Fella Records LLC/Def Jam B003X2O6KW, compact disc, 2010.

Young, Neil. Trans. Geffen B000024R1I, compact disc, 1995. Originally released in 1982. 